National

Academy

of

Sciences

National Research Council

NUCLEAR SCIENCE SERIES

Radiochemical Techniques

Processing of

Counting Data 


\section{COMMITTEE ON NUCLEAR SCIENCE}

D. A. Bromley, Chatrman

Yale University
R. D Evans, Vece Choirmar

Massachusetts Institute of Technology

Lewis Slack, Secretury

National Research Council

E. C. Anderson

Los Alamos Scientific Laboratory

N. E. Ballou

U. S. Naval Radiological Defense Laboratory

Martin J. Barger

National Bureau of Standards

C. J. Borkowsiki

Oak Ridge National Laboratory

Herbert Goldstein

Columbia University

Bernd Kahn

Taft Sanitary Engineering Center
Jerry B. Marion

University of Maryland

R. L. Platman

Argonne National Laboratory

Ernest C. Pollard

Pennsylvania State University

Katharine Way

Oak Pidge National Laboratory

George W. Wetherill

University of Caltfornia

Marvin E, Wyman

University of Ilinois

LUASON MEMBERS

Harold Glaser

Olfice of Naval Research
William S. Rodney

National Science Foundation

George A. Kolstad

Atomic Energy Commission

SUBCOMMITTEE ON RADIOCHEMISIRY

Nathan E. Ballou, Chairman

U. S. Naval Radiological Defense

$$
\text { Laboratory }
$$

G. R. Choppin

Florida State University

Herbert M. Clark

Rensselaer Polytechnic Institute

Richard M. Diamond

Lawrence Padiation Laboratory

Jerome Hudis

Brookhaven National Laboratory

Jere D. Knight

Los Alamos Scientific Laboratory

W. E. Nervik

Lawrence Radiation Laboratory
Jultan M. Nielsen

Battelle Pacific Northwest

G. D. O'Kelley

Oak Ridge National Laboratory

E. P. Steinberg

Argonne National Laboratory

D. N. Sunderman

Battelle Memorial Institute

John W. Winchester

Massachusetts Institute of Technology

R. P. Sehuman, Consultant

Sri Venkateswara University

Tirupati, Andhra Pradesh, Indiz 


\section{DISCLAIMER}

This report was prepared as an account of work sponsored by an agency of the United States Government. Neither the United States Government nor any agency Thereof, nor any of their employees, makes any warranty, express or implied, or assumes any legal liability or responsibility for the accuracy, completeness, or usefulness of any information, apparatus, product, or process disclosed, or represents that its use would not infringe privately owned rights. Reference herein to any specific commercial product, process, or service by trade name, trademark, manufacturer, or otherwise does not necessarily constitute or imply its endorsement, recommendation, or favoring by the United States Government or any agency thereof. The views and opinions of authors expressed herein do not necessarily state or reflect those of the United States Government or any agency thereof. 


\section{DISCLAIMER}

Portions of this document may be illegible in electronic image products. Images are produced from the best available original document. 


\title{
Processing of Counting Data
}

\author{
P. C. Stevenson \\ UNIVERSITY OF CALIFORNIA \\ Lawrence Radiation Laboratory \\ Livermore, Calıfornia
}

Issued: May 1966

Subcommittee on Radiochemistry

National Academy of Sciences - National Research Councll

Printed in USA. Price $\$ 1.75$. Avalable from the Clearinghouse for Federal Scientific and Technical Information, National Bureau of Standards, U.S. Department of Commerce, Springfield, Virginia. 
O

- 
FOREWORD

The Subcomittee on Radiochemistry is one of a number of subcomittees working under the Committee on Nuclear Science within the National Academy of Sciences - National Research Council. Its members represent government, industrial, and university laboratories in the areas of radiochemistry and nuclear chemistry. Support for the activities of this and other subcomittees of the Committee on Nuclear Science is provided by a grant from the National Science Foundation.

The Subcomittee has concerned itself with preparation of publications, encouraging and supporting activities in nuclear education, sponsoring symposia on selected current topics in radiochemistry and nuclear chemistry, and investigating special problems as they arise. A series of monographs on the radiochemistry of essentially all the elements and on radiochemical techniques is being published. Initiation and encouragement of publication of articles on nuclear education in various subject areas of chemistry have occurred, and development and improvement of certain educational activities (e.g., laboratory and demonstration experiments with radioactivity) have been encouraged and assisted. Radioactive contamination of reagents and materials has been investigated and specific recommendations made.

This series of monographs has resulted from the need for comprehensive compilations of radiochemical and nuclear chemical information. Each monograph collects in one volume the pertinent information required for radiochemical work with an individual element or with a specialized technique. The U. S. Atomic Energy Commission has sponsored the printing of the series.

Comments and suggestions for further publications and activities of value to persons working with radioactivity are welcomed by the Subcomittee. 
In this monograph I have attempted to collect a number of problems, and their solutions, which are of importance to radiochemists who are involved with the collection, reduction, and interpretation of various kinds of data arising from measurements using counting equipment.

In cases where the treatment of a problem has been taken from another author, I have endeavored to give full credit to the original source; errors in transcription or rephrasing are entirely my own. When no credit is given, I accept responsibility for the treatment and for the unhappiness which some of my procedures will cause more qualified statisticlans. My only defense is that I am attempting to write in a language which will be comprehensible to chemists.

This monograph has been prepared at the request of the Subcommittee on Radiochemistry of the Committee on Nuclear Science, Division of Physical Sciences, National Academy of Sciences - National Research Council, as one of the series of monographs on the techniques of radiochemistry which the Subcomittee is sponsoring. I would like to express my appreciation to the members of the Subcomittee for their encouragement; to Drs. E. Kaplan and H. B. Levy for their helpful discussions of the problems which arose during preparation of the manuscript; to the secretarial staff of the Radiochemistry Division and to the Technical Information Division of the Lawrence Radiation Laboratory for their assistance in preparing the manuscript.

This work was performed under the auspices of the U. S. Atomic Energy Commission.

P. C. Stevenson

Lawrence Radiation Laboratory

University of California

Livermore, Califormia 
Foreword

I. Introduction .......

1. General contents . . . . . . . . . . . . 1

II. Mathematics of Radioactive Processes . . . . . . 3

1. Assumptions . . . . . . . . . . . 3

2. Fundamental law of radioactive decay . . . . . 3

3. Formation of species in nuclear reactions . . . 5

4. Consecutive disintegrations $\quad . \quad$. $\quad . \quad$. $\quad 10$

5. Use of daughter activities to measure nuclear
reactions . . . . . . . . . . 13

6. Branching decay . . . . . . . . . 19

7. Decay chains with more than two consecutive decays . 21

III. Mathematical Techniques . . . . . . . . 25

]. Nature of the problem . . . . . . . . 25

2. Matrix algebra . . . . . . . . . 26

3. Statistical mathematics . . . . . . . . . 31

4. Averaging and sampling statistics . . . . . 39

5. The least-squares solution . . . . . . . 47

IV. Statistics of Counting Data . . . . . . . . 64

1. Nature of the problems . . . . . . . 64

2. Distribution functions of counting results . . . 64

3. Distribution of expected values for a given observed count - small sampling . . . . . . . 68

4. Distribution of expected values for a given observed count - large sampling . . . . . . . 69

5. Correction for counter background . . . . . . 76

6. Errors of extrapolation . . . . . . . . 79

7. Statistical reliability of measurements on nuclear
reactions . . . . . . . . . . . . . . .

V. The Resolution of Decay Data . . . . . . . . . $\quad$. 83

]. Nature of the problem . . . . . . . 83

2. The least-squares solution; weighting of the data . 84

3. Estimation of variance due to instrumental variation . 87 


\section{TABLE OF CONTENTS (Continued)}

Page No.

4. Effect of input parameters on resolution . . . $\quad 89$

5. Criteria for the rejection of data $\quad . \quad$. $\quad . \quad$. $\quad . \quad 92$

6. Avoidance of computer overflow . . . . . . . 94

7. Analysis of replicate samples; analysis of variance . 95

VI. The Identification of Components and Measurement of

Half-Lives or Decay Constants . . . . . . 101

1. Nature of the problems . . . . . . . . . 101

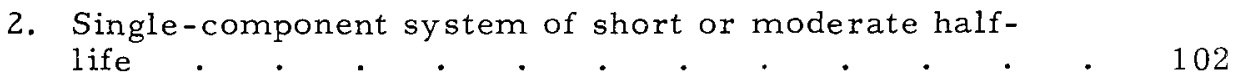

3. Single-component system with long half-life . . . 103

4. Multicomponent systems - déection . . . . 105

5. Known multicomponent systems - evaluation of $\lambda .107$

6. Unknown multicomponent systems . . . . . 109

VII. Dead Time and Coincidence Considerations . • . . . 110

]. Nature of the problem . . . . . . . 110

2. Effect of dead time on observed counting rate . . 112

3. Measurement of dead time . . . . . . . 114

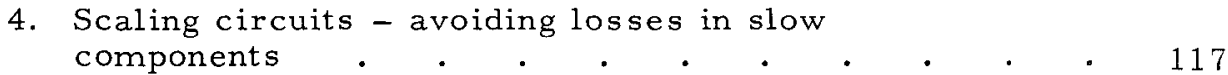

5. Measurement of coincident events . • . • . 120

6. Variance of coincident event rate . . . . . 123

7. The effect of coincident events on discriminated counts . . . . . . . . . . . . 124

8. Measurement of absolute counting efficiency by
coincidence techniques $\quad . \quad . \quad . \quad . \quad . \quad 127$

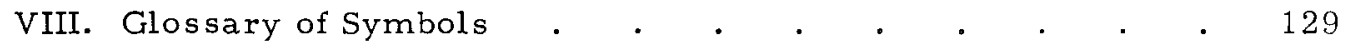

IX. Index $. \quad . \quad . \quad . \quad . \quad . \quad . \quad . \quad . \quad . \quad . \quad . \quad 131$ 


\title{
Processing of Counting Data
}

\author{
P. C. Stevenson \\ Lawrence Radiation Laboratory, University of California \\ Livermore, California
}

September 24, 1965

\section{INTRODUCTION}

\section{General Contents}

Counting data is the term which I propose to use loosely to describe the great variety of results of measurements on amount and kind of radiation emanating from a radioactive sample. In general, counting data are taken as a function of time after a certain preassigned zero time in terms of events per unit time. The instruments used to accumulate such data are many and various. They have been described in a number of publications (for a simple summary see another monograph of this series ${ }^{1}$ ). The interpretation and use of such data involve a number of complications, many of a statistical nature. This monograph is intended to be a handy reference manual of various ways of treating and interpreting data. It is also intended to serve as a reminder of some neglected aspects of the treatment of data and as a collection of information for those who are not familiar with the field.

Chapter II, Mathematics of Radioactive Processes, is concerned with the phenomenology of radiation: with its decay with time; with the formation of radioactive species by nuclear reactions; with consecutive processes (consecutive formations or consecutive decays) and competitive processes; and with the relationship of the parameters of nuclear reactions and nuclear processes to the raw data.

Chapter III on Mathematical Techniques gives a very elementary outline of the minimum necessary mathematics, matrix algebra, elementary statistical mathematics, a description of the least-squares method of curve fitting, and of the $x^{2}$ test as a criterion of goodness of fit. There is also a

\footnotetext{
${ }^{1}$ G. D. O'Kelley, "Detection and Measurement of Nuclear Radiation," NAS-NS-3105.
} 
description of a so-called " $F$ " test which is used to aid in making a decision whether the form of equation being fitted to the data is proper.

Chapter IV describes the statistical problems particularly associated with counting data. Variance of a single counting measurement is discussed in some detail. The effect of making a measurement involving small sampling, which is the usual method of procedure, is described and is followed by a description of the alterations in the treatment which should be used when large sampling or low count rates are involved. Statistical problems raised by correction for background are discussed, as are errors of extrapolation.

Chapter $\mathrm{V}$ is a detailed description of resolution methods applied to decay data, which involve separating a given set of data into components each attributable to a particular radioactive species. Proper weighting for least-squares analysis is discussed. There is a description of sources of variance and of the effect of input parameters such as half-lives. Criteria for data rejection are discussed, as are analyses of replicate samples.

Chapter VI is a description of the problems involved in identification of the components of the system and measurement of their decay characteristics; in particular, their half-lives. The problem of purity is discussed. The problem of measurement of half-life of single-component systems of short half-life and of long half-life is considered in some detail. Another problem considered in this section is the adjustment of half-lives with the best possible precision in a known multicomponent system where the halflives are not well known. This section also discusses the detection of multicomponent systems, the determination of the number of components in a system, and the measurement of the half-lives where the system components are completely unknown.

The last section discusses problems raised by the existence of dead time and coincidence phenomena. Dead time losses in counters are discussed. Live timers, which are mechanical methods of compensating for dead time loss, are mentioned. Regularizing action of scaling circuits is discussed in some detail. Measurement of coincident events and the problems raised by chance coincidences are discussed and the effect of coincidences on discriminated counts are considered. Finally the measurement of absolute counting efficiency by coincidence techniques is mentioned briefly. 


\section{MATHEMATICS OF RADIOACTIVE PROCESSES}

\section{As sumptions}

It will be assumed in this section that: (a) the intensity of radiation emerging from a sample of radioactive material can be measured with high precision and with known, or at least reproducible, efficiency; (b) that all atoms of a single radioactive species are identical in their nuclear properties; (c) that the intensity of the radiation originating in the sample is due to the nuclear properties of the species present; and (d) that each radioactive event signals the disintegration of one atom of the radioactive species and the formation of one atom of a new species. Assumptions (b), (c), and (d) have been verified experimentally; as sumption (a) is adopted in this section for simplicity to represent an ideal case and will be discarded in later sections.

\section{Fundamental Law of Radioactive Decay}

The assumptions listed above lead to the obvious conclusion that the amount of radiation originating in a sample is directly proportional to the amount of radioactive material present (although the constant of proportionality varies from species to species). For a single species, if $x$ is the number of atoms present at time $t$, the intensity of radiation in events per unit time is given by the rate at which the active species is disappearing (assumption (d)), so that the intensity of radiation is

$$
-\frac{d x}{d t}=+\lambda x
$$

where $\lambda$ is the constant of proportionality characteristic of the species. $\lambda$ is referred to as the decay constant of the species. The negative sign in the equation arises from assumption (d) above, that one radioactive event corresponds to the disappearance of one atom of the species. (It should be noted that we are ignoring the discrete nature of atomic matter in this section and treating $\mathrm{x}$ as a continuous variable. This is consistent with our first assumption.) Equation II.l represents one statement of the fundamental law of radioactive decay. It can be integrated immediztely to give

$$
x=x_{0} e^{-\lambda\left(t-t_{0}\right)}
$$


where $x$ has the value $x_{0}$ at the arbitrarily chosen time $t_{0}$. The product $\lambda x$ can be seen from equation II.l to represent the disintegration rate of the species; if we measure the radiation from the sample with an instrument having an efficiency $\eta$ for the characteristic radiations of our species, the observed "counting rate" will be given by $\eta \lambda x$. Both $x$ and $\eta \lambda x$ can be seen from equation II.2 to decrease exponentially with time.

A convenient measurement for purposes of discussion and observation is the time interval required for the activity of the species in the sample to decrease to one-half of its initial value. This interval $\mathrm{T}_{1 / 2}$ is referred to as the half-life, half-period, or simply period. Its value in terms of $\lambda$ can be derived by setting $x$ equal to $x_{0} / 2$ in equation II. 2 , so that

$$
e^{-\lambda T} 1 / 2=\frac{1}{2}
$$

and therefore

$$
\mathrm{T}_{1 / 2}=\frac{\ln 2}{\lambda} \text {. }
$$

$\mathrm{T}_{1 / 2}$ is expressed in time units and may be specified in units of any convenient size. $\lambda$ is expressed in units of reciprocal time; the nature of the units used is of particular significance when calculating disintegration rates from numbers of atoms or the reverse, since $\lambda$ and $\lambda x$ must be expressed in the same units.

If more than one species are present, the effects are additive, that is

$$
\text { Counting rate }=\sum_{i} \eta_{i} \lambda_{i} x_{i}=\sum_{i} \eta_{i} \lambda_{i} x_{i 0} e^{-\lambda_{i} t\left(t-t_{0}\right)}
$$

In such a system, each separate species is referred to as a component. The process of identifying the various components in an experimentally obtained set of data and of separating the effect due to each is called resolving the decay data. Information to be obtained in an experiment on radioactive material is usually gained from a measurement of the amount, the half-life, or the nature of the radiations of one or more components separately; it is with the resolution of multicomponent systems that the greater part of this paper will deal. 
The most elementary technique for resolving data on radioactıve decay depends on the fact that in any multicomponent mixture, there is usually one component much longer lived than any of the others. At late times, therefore, the activity still remaining in the system is likely to be due to a single long-lived component. If the activity of the system at various times is plotted as ordinate against time as abscissa on semilogarithmic graph paper (see Fig. II 1), and a smooth curve drawn through the points, the last portion of the curve obtained will frequently be a good approximation to a straight line. (If only one component is present, the entıre curve will be a straight line.) This straight line can be extrapolated back to the times of the early observations and the contribution of the longest lived species subtracted from each gross amount. The differences are then replotted and the procedure repeated for the next-longest lived species. This technique is simple, but not very precise and inevitably prone to subjective judgments.

The straight lines obtained from the above-described plots may be used to get an estimate of the half-lives of the components by observing the time required for the amount of each component to decrease by exactly a factor of 2, since the linear nature of the plots makes interpolation to the desıred actıvity level particularly simple. Somewhat better precision may be obtaıned by observing the time required for the activity to decrease by an integral power of 2 , and dividing that time by the power used.

\section{Formation of Species in Nuclear Reactions ${ }^{2}$}

Radioactive species are formed either by nuclear reactions between nuclei and subatomic partıcles or photons or by spontaneous radioactıve decay of other radioactive parent species. In this section we shall consider the number of atoms $\mathrm{x}$ of a radioactive species present in a sample of material exposed to conditions leading to the formation of the radioactive species, e.g., in a cyclotron beam or a flux of neutrons in a reactor.

Let the flux of particles at each atom in the target material be $\phi$ The rate of the nuclear reaction will be proportional to the flux integrated over the entire sample. If the flux is uniform, this is simply the product of the flux $\phi$ by the number $n$ of atoms in the sample, if the flux is limited to a beam passing through a sample of unform thickness, it is the product of the total beam current by the thickness of the sample in atoms per unit area. In actual practice the flux is usually not uniform, gradients in the flux in a sample of finıte size may arıse either from the gradient in space of the flux into which the sample is inserted or from the attenuation of the flux by the sample itself. One must then integrate the flux at each infinitesimal volume element, times the number of atoms of target in that volume element, over the volume of the entire target. In

\footnotetext{
${ }^{2}$ See Rubinson, J Chem Phys. 17, 542 (1949)
} 

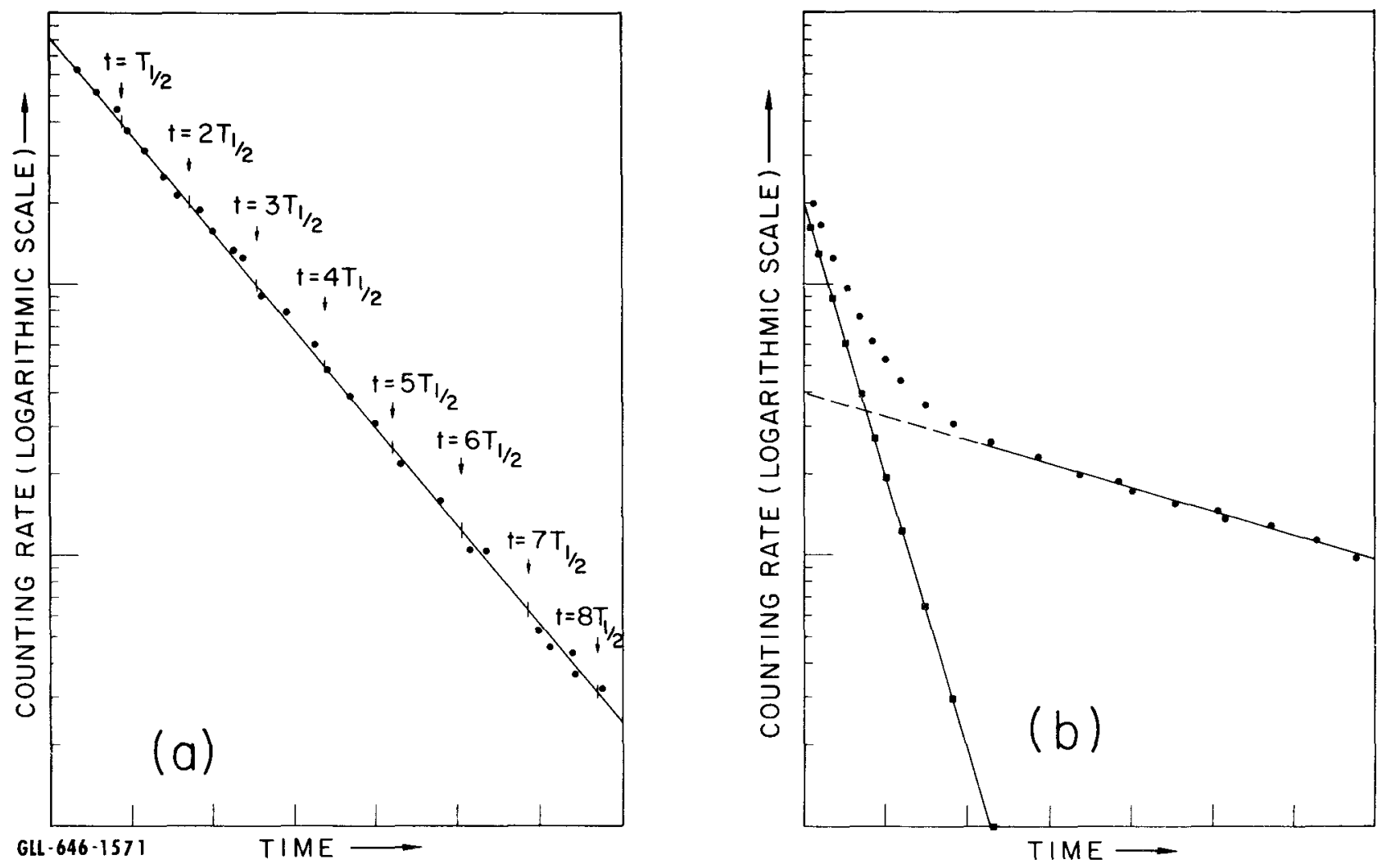

Fig. II.1. (a) Activity of a single-component radioactive sample plotted against time. Arrows mark the points on the line at which the activity has decreased by successive factors of 2 . Time interval between arrows is one half-life. The best graphical estimate of the half-life is obtained by dividing the

time interval between the zero time and the time of the nth arrow by $n$, where $n$ is as great as possible. No real advantage is gained by extrapolating the curve beyond the measured data, although it is convenient to extrapolate to the next even half-life.

(b) Activity of a two-component radioactive sample plotted against time. Circles are measured data points. Squares are obtained by subtracting the value of the extrapolated long-lived activity (dashed curve) from each measurement. Half-lives of the two species differ by approximately a factor of 10 . 
either case, we shall use the symbolic product no to represent this integral. We shall define a reaction cross section $\sigma$ to be the constant of proportionality which converts the product $n \phi$ to the actual rate of the nuclear reaction. It is apparent that if we consider the reaction to be occurring between an assembly of flying particles and a collection of atoms, then $\sigma$ is simply the "target area" presented to the particles by a single atom.

The net rate of formation, however, contains another term, since the species being formed is radioactive and hence subject to the laws governing radioactive decay. The expression for the net rate of formation of the species is therefore given by

$$
\frac{d x}{d t}=n \phi \sigma-\lambda x
$$

where the second term on the right arises from the decay laws, expressed in the form of equation II.l. In most cases, no may be considered constant, since most methods of inducing nuclear reactions are extremely inefficient in terms of conversion of the target material. If this assumption is made, it is obvious that, whatever the starting conditions, the system will tend in the long $r$ un to an equilibrium value of $x$, since for small $x$ the derivative is positive so that $x$ is increasing, while for large $x$ the derivative is negative so that $\mathrm{x}$ is decreasing. When the system has its equilibrium value of $\mathrm{x}$, it is said to be saturated.

If $n \phi$ does not change with time, equation II.6a can readily be integrated by rearranging the terms and applying an integrating factor, as follows:

$$
\begin{aligned}
& \frac{d x}{d t}+\lambda x=n \phi \sigma, \\
& e^{\lambda t}\left(\frac{d x}{d t}+\lambda x\right)=n \phi \sigma e^{\lambda t}, \\
& \frac{d}{d t}\left(x e^{\lambda t}\right)=n \phi \sigma e^{\lambda t}, \\
& x e^{\lambda t}-x_{0} e^{\lambda t_{0}}=\frac{n \phi \sigma}{\lambda}\left(e^{\lambda t}-e^{\lambda t_{0}}\right),
\end{aligned}
$$

and finally,

$$
x=x_{0} e^{-\lambda\left(t-t_{0}\right)}+\frac{n \phi \sigma}{\lambda}\left[1-e^{-\lambda\left(t-t_{0}\right)}\right] .
$$


In this equation the first term represents the behavior of the radioactive material present at the time $t_{0}$, decaying away according to equation II.2, while the second term represents the buildup toward equilibrium of the material formed by nuclear reactions in the time following $t_{0}$.

Equation II.7 only applies as long as the reaction is continuing; when the cyclotron beam is turned off or the sample is removed from the reactor, the active material present begins to decay away, following equation II.2 with $t_{0}$ for that equation being taken as the time of the end of the irradiation. The counting rate measured at some later time can always be extrapolated back to the end of the irradiation if the half-life is known; in the subsequent discussion it will be assumed that this has been done.

If $n \phi$ is not constant with time, equation II. 7 cannot be obtained in analytic form unless the dependence of $n \phi$ on $t$ is expressed analytically. Treatment of equation II.6a proceeds as before up to the integration step II.6e, which then becomes

$$
d\left(x e^{\lambda t}\right)=\sigma e^{\lambda t} n \phi(t) d t
$$

leading to

$$
x=x_{0} e^{-\lambda\left(t-t_{0}\right)}+\sigma e^{-\lambda t} \int_{t_{0}}^{t} n \phi(t) e^{\lambda t} d t
$$

which usually must be evaluated numerically. In what follows we shall assume that flux at any point in the target is constant with time.

In a typical experiment, inactive material is ir radiated at a constant rate for a defined length of time in a constant flux; $x_{0}$ is zero and equation II. 7 can be used to give a measurement of $\phi$ if $\sigma$ is known, or of $\sigma$ if $\phi$ is known:

$$
\begin{aligned}
& x=\frac{\phi n \sigma}{\lambda}\left[1-e^{-\lambda\left(t-t_{0}\right)}\right], \\
& \sigma=\frac{1}{\phi n} \frac{\lambda x}{\left[1-e^{-\lambda\left(t-t_{0}\right)}\right]}=\frac{1}{\eta \phi n} \frac{\text { counting rate }}{\left[1-e^{-\lambda\left(t-t_{0}\right)}\right]} \\
& \phi=\frac{1}{\sigma n} \frac{\lambda x}{\left[1-e^{-\lambda\left(t-t_{0}\right)}\right]}=\frac{1}{\eta \sigma n} \frac{\text { counting rate }}{\left[1-e^{-\lambda\left(t-t_{0}\right)}\right]} .
\end{aligned}
$$


In either case the detector efficiency $\eta$ must be known, which is frequently not the case; if $\eta$ is not known relative numbers can be obtained, since $\eta$ does not vary from experiment to experiment if the equipment is reliable.

If a calibration experiment is made with a known amount of material in a uniform flux, a useful "figure of merit" $\eta \phi \sigma$ may be obtained:

$$
\eta \phi \sigma=\frac{\text { counting rate }}{n\left[1-e^{-\lambda\left(t-t_{0}\right)}\right]}=a \text {. }
$$

This figure of merit may then be applied to irradiations of unknown materials in the same flux to determine the amount of the target species in the unknown sample, since

$$
\mathrm{n}=\frac{\text { counting rate }}{a\left[1-\mathrm{e}^{-\lambda(\mathrm{t}-\mathrm{t})}\right]} \text {. }
$$

This is the basis of the very sensitive analytical method known as activation analysis. Care must be taken to reproduce the flux exactly; in practice, the calibration sample and the unknown are usually run simultaneously.

Returning to equation II.6a, it is sometimes necessary to modify our simplifying assumption. Development of high-flux reactors and other devices which are relatively efficient, though rather spectacularly shortlived, render it necessary to consider the case in which the amount of target material is appreciably depleted during the course of the experiment. For simplicity, we shall as sume that the flux in this case is constant and uniform. We then have, since formation of one atom of $\mathrm{x}$ implies destruction of one atom of $\mathrm{n}$,

$$
\frac{\mathrm{dn}}{\mathrm{dt}}=-\phi \mathrm{n} \sigma
$$

so that

$$
n=n_{0} e^{-\phi \sigma\left(t-t_{0}\right)}
$$

which may be substituted into equation II.6a. Following a method of integration analogous to that used on equation II.6a, we finally get 


$$
x=x_{0} e^{-\lambda\left(t-t_{0}\right)}+\frac{\phi n_{0} \sigma e^{-\phi \sigma\left(t-t_{0}\right)}}{\lambda-\phi \sigma}\left[1-e^{-(\lambda-\phi \sigma)\left(t-t_{0}\right)}\right] .
$$

After very long irradiation times, this expression tends toward zero, owing to complete conversion of the target material followed by radioactive decay of the product. If we maximize $x$ with respect to time, we can easily see that the greatest amount of product is present in the target at a time given by (assuming $x_{0}=0$ )

$$
t_{m}-t_{0}=\frac{\ln (\lambda / \phi \sigma)}{\lambda-\phi \sigma}
$$

Finally, if the product itself reacts with the flux, as it usually does, we must modify equation II.6a still further by addition of a term $-\phi \sigma_{x} x$ where $\sigma_{x}$ is the reaction cross section of the species $x$ with the flux. It is obvious that the only difference made in the mathematical form of the equation is the replacement of the constant $\lambda$ with a new constant $\lambda+\phi \sigma_{x}$. The integrated for $m$ of the equation is then readily obtained by substitution in equation II.13, and the time of maximum production by substitution in equation II.14, giving

$$
\begin{gathered}
x=x_{0} e^{-\left(\lambda+\phi \sigma_{x}\right)\left(t-t_{0}\right)}+\frac{\phi n_{0} \sigma e^{-\phi \sigma\left(t-t_{0}\right)}}{\lambda+\phi \sigma_{x}-\phi \sigma}\left[1-e^{-\left(\lambda+\phi \sigma_{x}-\phi \sigma\right)\left(t-t_{0}\right)}\right], \\
t_{m}-t_{0}=\frac{\ln \left(\frac{\lambda+\phi \sigma_{x}}{\lambda \sigma}\right)}{\lambda+\phi \sigma_{x}-\phi \sigma} .
\end{gathered}
$$

\section{Consecutive Disintegrations}

It frequently happens that the disintegration product of a radioactive species is itself radioactive. The mathematical analysis of the system is quite straightforward; it is only necessary to keep in mind, first that the fundamental law of decay applies to all active species, and second that disintegration of one atom of the "parent" species corresponds to formation of one atom of the "daughter" species.

Let us consider a simple system, a species 1 decaying to a species 2 which decays to a stable end product. We have for the parent species

$$
x_{1}=x_{10} e^{-\lambda_{1}\left(t-t_{0}\right)},
$$




$$
\eta_{1} \lambda_{1} x_{1}=\eta_{1} \lambda_{1} x_{10} e^{-\lambda_{1}\left(t-t_{0}\right)}
$$

for the amount and counting rate, respectively, of the parent species. The net rate of formation of the daughter species is given by

$$
\frac{d x_{2}}{d t}=-\lambda_{2} x_{2}+\lambda_{1} x_{1}=-\lambda_{2} x_{2}+\lambda_{1} x_{10} e^{-\lambda_{1}\left(t-t_{0}\right)} .
$$

Equation II. 18 may be integrated in a manner analogous to that used for equation II.6a to give

$$
x_{2}=x_{20} e^{-\lambda_{2}\left(t-t_{0}\right)}+\frac{\lambda_{1}}{\lambda_{2}-\lambda_{1}} x_{10}\left[e^{-\lambda_{1}\left(t-t_{0}\right)}-e^{-\lambda_{2}\left(t-t_{0}\right)}\right]
$$

$\eta_{2} \lambda_{2} x_{2}=\eta_{2} \lambda_{2} x_{20} e^{-\lambda_{2}\left(t-t_{0}\right)}+\frac{\eta_{2} \lambda_{1} \lambda_{2}}{\lambda_{2}-\lambda_{1}} x_{10}\left[e^{-\lambda_{1}\left(t-t_{0}\right)}-e^{-\lambda_{2}\left(t-t_{0}\right)}\right]$.

(In the highly unlikely event that $\lambda_{1}=\lambda_{2}=\lambda$, it is a simple matter to show that

$$
x_{2}=x_{20} e^{-\lambda\left(t-t_{0}\right)}+\lambda x_{10}\left(t-t_{0}\right) e^{-\lambda\left(t-t_{0}\right)}
$$

either by rederiving equation II. 19a or by extracting the factor $e^{-\lambda_{1}\left(t-t_{0}\right)}$ from the parenthesis in equation II. $19 a$ to leave a term $\left[1-e^{-\left(\lambda_{2}-\lambda_{1}\right)\left(t-t_{0}\right)}\right]$. This term approaches $\left(\lambda_{2}-\lambda_{1}\right)\left(t-t_{0}\right)$ as a limit as $\left(\lambda_{2}-\lambda_{1}\right)$ approaches zero. This case will not be considered further.)

The total counting rate of the sample is obtained by adding the rates given by equations II.17b and II.19b to give Counting rate $=\eta_{1} \lambda_{1} x_{10} e^{-\lambda_{1}\left(t-t_{0}\right)}+\eta_{2} \lambda_{2} x_{20} e^{-\lambda_{2}\left(t-t_{0}\right)}$

$$
+\frac{\eta_{2} \lambda_{1} \lambda_{2}}{\lambda_{2}-\lambda_{1}} x_{10}\left[\mathrm{e}^{-\lambda_{1}\left(t-t_{0}\right)}-e^{-\lambda_{2}\left(t-t_{0}\right)}\right]
$$

Combining terms with like exponential factors, we get 
Counting rate $=\lambda_{1} x_{10} e^{-\lambda_{1}\left(t-t_{0}\right)}\left[\frac{\lambda_{2}\left(\eta_{1}+\eta_{2}\right)-\lambda_{1} \eta_{1}}{\lambda_{2}-\lambda_{1}}\right]$

$$
+\eta_{2} \lambda_{2} e^{-\lambda_{2}\left(t-t_{0}\right)}\left[\frac{x_{20}\left(\lambda_{2}-\lambda_{1}\right)-\lambda_{1} x_{10}}{\lambda_{2}-\lambda_{1}}\right] .
$$

It can easily be seen from equation II.2 l that the decay of a parentdaughter system resembles that of any two-component system, and can be resolved on that basis. The half-lives of the apparent components are the actual half-lives of the parent and the daughter. However, when performing an experiment in which the product of interest gives rise to an active daughter, the presence of the daughter activity may lead to serious error unless an appropriate correction factor is applied. Let us assume that an irradiation is made and that a product (species 1 ) is separated which has an active daughter (species 2). The product is purified, the decay of the sample is followed for a time and the data resolved into its two components. We then attempt to apply equations II.10b or II.10c to the system, using the resolved counting rate of the component having the half-life of species 1 . At the time of purification of the parent species, equation II.2 I gives

$$
\text { Counting rate }(1)=\lambda_{1} x_{1}\left[\frac{\lambda_{2}\left(\eta_{1}+\eta_{2}\right)-\lambda_{1} \eta_{1}}{\lambda_{2}-\lambda_{1}}\right]
$$

while in the absence of the caughter the counting rate at the same time would be

$$
\text { Counting rate (no daughter) }=\lambda_{1} x_{1} \eta_{l} \text {. }
$$

The ratio of the observed to the desired rate is the growth factor $g$, which is independent of time (since both numerator and denominator in the ratio have the same time dependence) and consequently applies at the end of the ir radiation. It is given by

$$
g=1+\left(\frac{\lambda_{2}}{\lambda_{2}-\lambda_{1}}\right)\left(\frac{\eta_{2}}{\eta_{1}}\right) \text {. }
$$

Equations II. $10 \mathrm{~b}$ and II.10c apply to the counting rate in the absence of daughter activity; therefore the observed resolved counting rate must be divided by $g$ before being inserted in these equations. 
It will be observed that, unless $\eta_{2}$ is very small, as $\lambda_{2}$ approaches $\lambda_{1}$ the value of $\mathrm{g}$ becomes very large either positive or negative and is approximately proportional to the reciprocal of their difference. Since the values of the $\lambda$ 's are not in general known with extreme accuracy, the accuracy of their difference becomes extremely poor as that difference becomes small with respect to the $\lambda$ 's themselves. Hence in parentdaughter systems in which the two components have comparable half-lives, it is very desirable to count with an instrument which is as specific for the detection of the parent as possible. If this is not practicable, it may be advisable to base the experiment on a direct measurement of the daughter activity, as discussed in the next section.

It should be noted especially that when the daughter is considerably longer lived than the parent $\left(\lambda_{1}>\lambda_{2}\right)$, the value of $g$ is less than 1 , that is, that the presence of a long-lived daughter actually decreases the apparent activity of the parent (see Fig. II.2). It is well known that the presence of a short-lived daughter increases the apparent activity of the parent, since at equilibrium every disintegration of the parent is shortly followed by a disintegration of the daughter, but the opposite effect of a long-lived daughter is likely to be forgotten, since it is not nearly as obvious. Presence of a short-lived daughter will usually cause an actual increase in the counting rate at early times which is quite obviously different from a simple twocomponent decay; the decay of a system containing a long-lived daughter cannot be distinguished from that of a simple mixture of species.

\section{Use of Daughter Activities to Measure Nuclear Reactions}

It is sometimes convenient to obtain results from an experiment by analyzing, not for material formed directly, but for a daughter species which may be longer lived, less subject to errors or interferences, or more tractable chemically. In this case the interpretation of the results is straightforward, but requires some thought. We may define two separate cases: case l, in which the parent is purified and the daughter is allowed to grow into the purified sample, and then is separated and counted; and case 2 , in which the parent is not separated, but the daughter is separated directly from the irradiated material. 

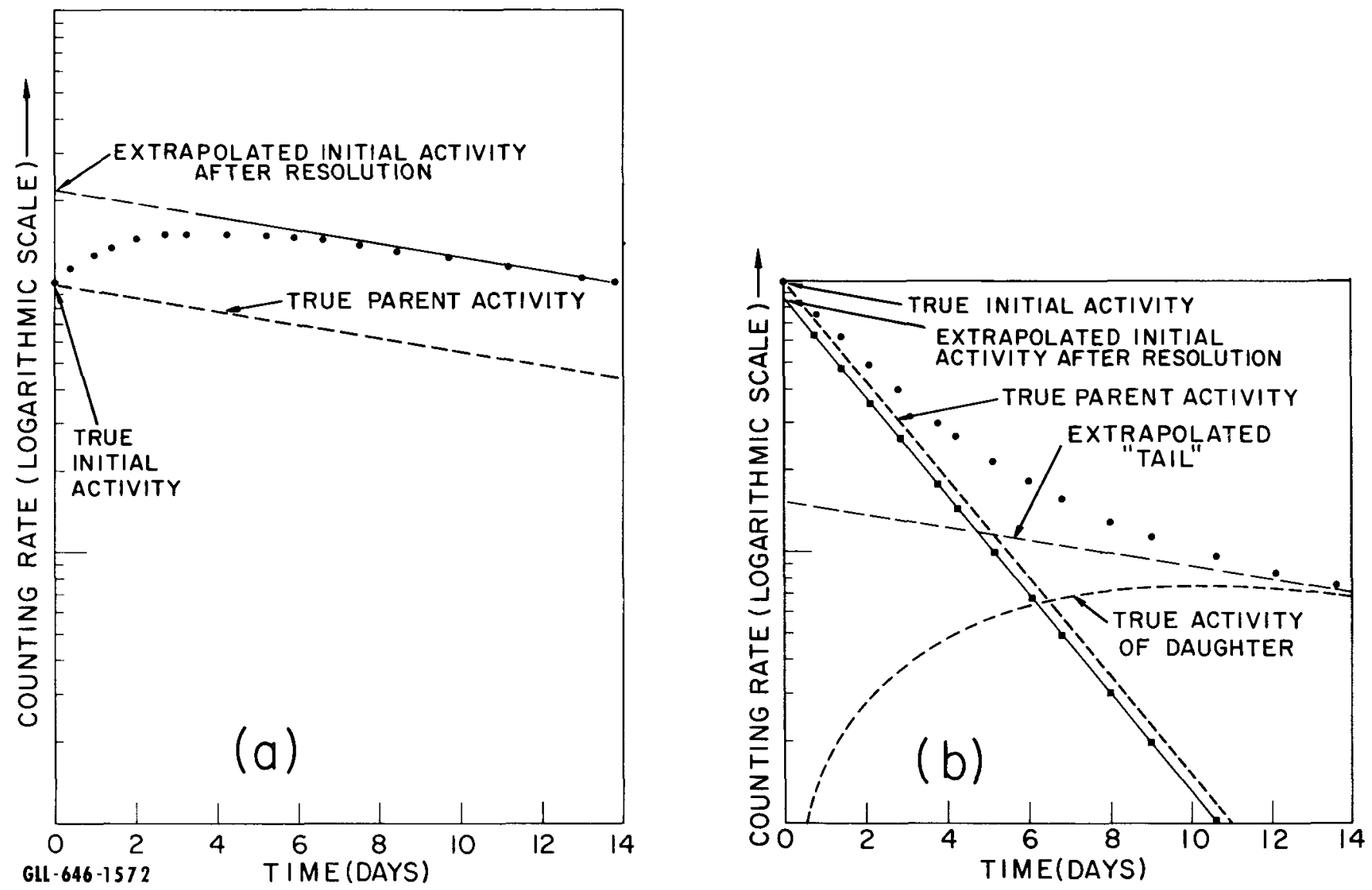

Fig. II.2. Curves showing the early part of the decay of a parent-daughter pair. In each case the halflives are 12.8 and 1.67 days; in (a) the longer-lived species is the parent, while in (b) the shorter-lived species is the parent. Equal counting efflciencies have been assumed. In neither case does the extrapolated "resolved parent activity" equal the true value. The ratio of observed to true value is given by the factor $g$ (equation II.24). Note especially that, for (b), $g$ is less than 1. 
Case 1: This technique is usually used when the half-life of the parent is much longer than that of the daughter. A typical example is the usual method for the analysis of $\mathrm{Sr}^{90}$. In ordinary fission product samples the radiations of $\mathrm{Sr}^{90}$ constitute a very small fraction of the total radiations of the strontium fraction. Furthermore, one of the competing species $\left(\mathrm{Sr}^{89}\right.$ ) has a half-life of almost two months, so that waiting for the shorter components to decay away, leaving relatively pure $\mathrm{Sr}^{90}$, is out of the question. The analysis may readily be performed, however, by separation and counting of the daughter species $\mathrm{Y}^{90}$, since $\mathrm{Sr}^{89}$ has no active daughter. It is well to remark that, prior to purification of the strontium fraction, it is necessary to wait for several days after the irradiation in order to permit the essentially complete decay of $\mathrm{Sr}^{91}$, which is initially present in very large amounts and which does have an active yttrium daughter, as will be discussed under case 2 below.

The analysis is carried out as follows:

1) At the end of the irradiation, the amount of the parent species is (from equation II. 10a)

$$
x_{10}=\frac{\phi n \sigma}{\lambda_{1}}\left(1-e^{-\lambda_{1} \Delta}\right)
$$

where $\Delta$ is the duration of the irradiation.

2) After a waiting period of duration $w$, the parent species is purified. The amount present at this time is (from equations II.25 and II.2)

$$
x_{1 w}=\frac{\phi n \sigma}{\lambda_{1}}\left(1-e^{-\lambda_{1} \Delta}\right) e^{-\lambda_{1} w} \text {. }
$$

3) The purified parent material is allowed to stand for a time to enable the daughter species to grow into the sample. The time $t$ is usually, though not always, long compared to the half-life of the daughter, since this simplifies the analysis of the results. After the time t has elapsed, the daughter species is purified and counted. The amount present at the time of purification is (from equations II.26 and II. 19a)

$$
x_{2}=\frac{\phi n \sigma}{\lambda_{2}-\lambda_{1}}\left(1-e^{-\lambda_{1} \Delta}\right) e^{-\lambda_{1} w}\left[e^{-\lambda_{1} t}-e^{-\lambda_{2} t}\right]
$$

and its counting rate is 
Counting rate $=\frac{\phi n \sigma \eta_{2} \lambda_{2}}{\lambda_{2}-\lambda_{1}}\left(1-e^{-\lambda_{1} \Delta}\right) e^{-\lambda_{1} w}\left(e^{-\lambda_{1} t}-e^{-\lambda_{2} t}\right)$

where, as usual, the counting rate is corrected to zero time - in this case, the time of separation from the parent.

In the ideal case, when the parent species is extremely long-lived and the growth period $t$ is long compared to the half-life of the daughter, the expression simplifies to

$$
\text { Counting rate }=\frac{\phi n \sigma \eta_{2} \lambda_{2}}{\lambda_{2}-\lambda_{1}} . \quad \text { (Ideal Case) }
$$

Equation II.27b can be rearranged to give information concerning $\phi$, $\sigma$, or $\mathrm{n}$ in the manner of equations II. $10 \mathrm{~b}$, II. $10 \mathrm{c}$, or II. $10 \mathrm{e}$.

Case 2: This technique is usually used when the species directly formed in the reaction of interest is relatively short-lived, decaying into a longer lived species which is more convenient to work with for any one of a number of reasons. A typical example is the usual analysis for $\mathrm{Sr}^{91}$ formed in fission. This species is most conveniently measured by analyzing for the daughter product $\mathrm{Y}^{91}$, which is longer lived and less subject to interference from isotopic activities. The analysis is carried out in a different manner from that described under case 1 , as follows:

1) During an irradiation of duration $\Delta$, the amount of the parent species builds up according to equation II. 10a, so that

$$
\mathrm{x}_{10}=\frac{\phi n \sigma}{\lambda_{1}}\left(1-\mathrm{e}^{-\lambda_{1} \Delta}\right) \text {. }
$$

The daughter species also builds up, in a manner to be derived as follows:

$$
\frac{d x_{2}}{d t}=-\lambda_{2} x_{2}+\lambda_{1} x_{1}=-\lambda_{2} x_{2}+\phi n \sigma\left(1-e^{-\lambda_{1} t}\right) .
$$

Using a method of integration like that used for equation II.6a, letting $t$ go from zero to $\Delta$ and letting $x_{20}$ be zero,

$\mathbf{x}_{2 \Delta}=\phi n \sigma\left[\frac{1-e^{-\lambda_{2} \Delta}}{\lambda_{2}}-\mathrm{e}^{-\lambda_{2} \Delta} \frac{1-\mathrm{e}^{-\left(\lambda_{1}-\lambda_{2}\right) \Delta}}{\lambda_{1}-\lambda_{2}}\right]$ 
2) The sample is allowed to wait for a time w and the daughter species is then purified and counted. In contrast to case 1 , the parent species is never purified. Equation II.19a then applies, with $x_{20}$ of II.19a replaced by $x_{2 \Delta}$ from equation II. $30, x_{10}$ obtained from equation II.28, and $t-t_{0}$ equal to $w$. This gives, at the purification time,

$$
\begin{aligned}
x_{2}=\phi n \sigma\left[\frac{1-e^{-\lambda_{2} \Delta}}{\lambda_{2}}-e^{-\lambda_{2} \Delta} \frac{1-e^{-\left(\lambda_{1}-\lambda_{2}\right) \Delta}}{\lambda_{1}-\lambda_{2}}\right] e^{-\lambda_{2} w} & \\
& +\frac{\phi n \sigma}{\lambda_{2}-\lambda_{1}}\left(1-e^{-\lambda_{1} \Delta}\right)\left(e^{-\lambda_{1} w}-e^{-\lambda_{2} w}\right) .
\end{aligned}
$$

If the daughter activity is extrapolated back to the end of the irradiation in the usual way, we get

$x_{2}=\phi n \sigma\left\{\frac{1-e^{-\lambda_{2} \Delta}}{\lambda_{2}}-e^{-\lambda_{2} \Delta} \frac{1-e^{-\left(\lambda_{1}-\lambda_{2}\right) \Delta}}{\lambda_{1}-\lambda_{2}}+\frac{\left(1-e^{-\lambda_{1} \Delta}\right)\left[1-e^{-\left(\lambda_{1}-\lambda_{2}\right) w}\right]}{\lambda_{1}-\lambda_{2}}\right\}$

and

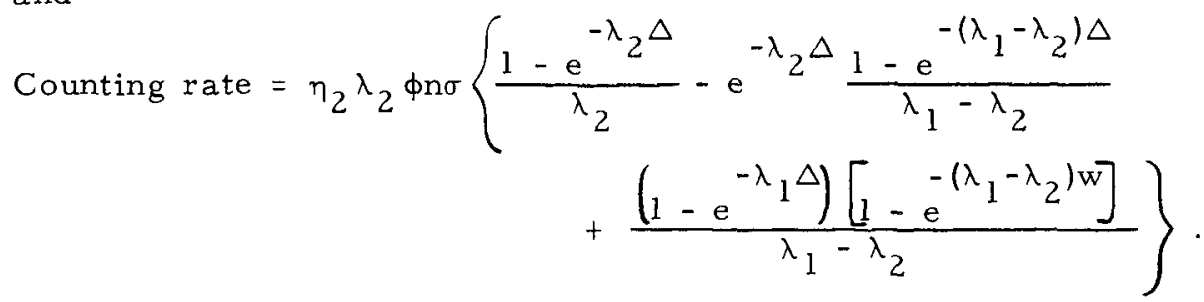

If $\lambda_{1}$ is sufficiently large compared to $\lambda_{2}$, and $w$ is sufficiently large compared to the parent half-life, then equation II.32b is well approximated by equation II.10a. This demonstrates that formation of a species by way of a negligibly short intermediate species is not appreciably different from direct formation. It should be noted that equations II. $32 \mathrm{~b}$ and II. $27 \mathrm{~b}$ are not equivalent.

An important subcase of case 2 should also be considered. It sometimes happens, for example in the formation of the primary fission products or in reactions where the product formed has a metastable isomeric state, that both the parent and the daughter species are formed directly by nuclear reactions. Analysis of the data in such a case is far from simple. Equation II. 29 must be modified to 


$$
\frac{\mathrm{d} \mathbf{x}_{2}}{\mathrm{dt}}=-\lambda_{2} \mathrm{x}_{2}+\phi \mathrm{n}_{1}\left(1-\mathrm{e}^{-\lambda_{1} \mathrm{t}}\right)+\phi \mathrm{n} \sigma_{2}
$$

which upon integration gives

$\mathrm{x}_{2}=\phi n\left[\left(\sigma_{1}+\sigma_{2}\right) \frac{1-\mathrm{e}^{-\lambda_{2} \Delta}}{\lambda_{2}}-\sigma_{1} e^{-\lambda_{2} \Delta} \frac{1-\mathrm{e}^{-\left(\lambda_{1}-\lambda_{2}\right) \Delta}}{\lambda_{1}-\lambda_{2}}\right]$.

Treating the sample in the same manner as before, we arrive at the results

$\begin{aligned} \mathbf{x}_{2}=\operatorname{\phi n}\left\{\left(\sigma_{1}+\sigma_{2}\right) \frac{1-\mathrm{e}^{-\lambda_{2} \Delta}}{\lambda_{2}}-\sigma_{1} \mathrm{e}^{-\lambda_{2} \Delta} \frac{1-\mathrm{e}^{-\left(\lambda_{1}-\lambda_{2}\right) \Delta}}{\lambda_{1}-\lambda_{2}}\right. & \\ & \left.+\sigma_{1} \frac{\left(1-\mathrm{e}^{-\lambda_{1} \Delta}\right)\left[1-\mathrm{e}^{-\left(\lambda_{1}-\lambda_{2}\right) \mathrm{w}}\right]}{\lambda_{1}-\lambda_{2}}\right\},\end{aligned}$

Counting rate $=\eta_{2} \lambda_{2} \phi n\left\{\left(\sigma_{1}+\sigma_{2}\right) \frac{1-e^{-\lambda_{2} \Delta}}{\lambda_{2}}-\sigma_{1} e^{-\lambda_{2} \Delta} \frac{1-e^{-\left(\lambda_{1}-\lambda_{2}\right) \Delta}}{\lambda_{1}-\lambda_{2}}\right.$

$$
\left.+\sigma_{1} \frac{\left(1-e^{-\lambda_{1} \Delta}\right)\left[1-e^{-\left(\lambda_{1}-\lambda_{2}\right) w}\right]}{\lambda_{1}-\lambda_{2}}\right\}
$$

We then see that if the experiment is intended to give a measurement of cross section, two runs must be made with times $\triangle$ and $w$ selected to give substantially different values of the exponential factor, since two separate cross sections are involved; furthermore, we see that unless the later terms in the bracket are negligibly small compared to the first term (which, for comparable cross sections, will only happen if $\lambda_{1} \gg \lambda_{2}$, that is, for a very short-lived parent), the effective reaction cross section is not simply the sum of the two cross sections involved, but depends on the time schedule of the experiment in a rather elaborate fashion. In cases where the half-lives are comparable to each other and to $w$ and $\Delta$, the errors caused by this effect may be large indeed.

If the two species concerned are nuclear isomers, purification of the daughter from the parent is not usually practical in an analytical procedure. In this case equation II.35b still applies, with the value of $w$ taken as infinite. 
It is also of interest to note that if both species can be counted, either in the same sample or separately, then the counting rate of the parent species furnishes a measure of $\sigma_{1}$ if equations II.24 and II. $10 \mathrm{~b}$ are used. This value may then be used in equation II. $35 \mathrm{~b}$ to permit the value of $\sigma_{2}$ to be calculated.

\section{Branching Decay}

Some radioactive species may follow either of two decay paths to produce either of two decay products. (Am ${ }^{242}$, indeed, follows three!) The ratio between the rates of the two kinds of disintegrations is fixed and characteristic of the species, and is known as the branching ratio. Many examples are known in the natural radioactive series $-\mathrm{UX}_{1}\left(\mathrm{Th}^{23}\right)$ may decay by beta emission to either one of the two isomers $\mathrm{UX}_{2}\left(\mathrm{~Pa}^{231 \mathrm{~m}}\right)$ or $\mathrm{UZ}\left(\mathrm{Pa}^{231}\right)$; or $\mathrm{RaC}\left(\mathrm{Bi}^{214}\right)$ may decay by beta emission to $\mathrm{RaC}^{\prime}\left(\mathrm{Po}^{214}\right.$ ) or by alpha emission to $\mathrm{RaC} "\left(\mathrm{Tl}^{210}\right)$. This behavior may be described mathematically by separating the decay constant $\lambda$ of equation II. 1 into two partial decay constants, $\lambda_{1 a}$ and $\lambda_{1 b}$, whose ratio is the branching ratio.

There is no effect of branching decay on the time dependence of the counting rate if neithe $r$ of the daughter species is active, although the counter efficiency is no longer simple:

$$
\eta_{1}=\frac{\lambda_{1 a}}{\lambda_{1 a}+\lambda_{1 b}} \eta_{1 a}+\frac{\lambda_{1 b}}{\lambda_{1 a}+\lambda_{1 b}} \eta_{1 b} .
$$

If the daughter species are active, equations II. 1 and II. 18 become

$$
\begin{aligned}
& \frac{d x_{1}}{d t}=-\left(\lambda_{1 a}+\lambda_{1 b}\right) x_{1}, \\
& \frac{d x_{2 a}}{d t}=-\lambda_{2 a} x_{2 a}+\lambda_{1 a} x_{1}, \\
& \frac{d x_{2 b}}{d t}=-\lambda_{2 b} x_{2 b}+\lambda_{1 b} x_{l} .
\end{aligned}
$$

Upon integration, these equations give

$$
x_{1}=x_{10} e^{-\left(\lambda_{1 a}+\lambda_{1 b}\right)\left(t-t_{0}\right)} \text {, }
$$




$$
\begin{aligned}
& x_{2 a}=x_{2 a 0} e^{-\lambda_{2 a}\left(t-t_{0}\right)}+\frac{\lambda_{1 a} x_{10}}{\lambda_{2 a}-\lambda_{1 a}-\lambda_{1 b}}\left[e^{-\left(\lambda_{1 a}+\lambda_{1 b}\right)\left(t-t_{0}\right)}\right. \\
& x_{2 b}=x_{2 b 0} e^{-\lambda_{2 b}\left(t-t_{0}\right)}+\frac{\lambda_{2 b} x_{10}}{\lambda_{2 b}-\lambda_{1 a}-\lambda_{1 b}}\left[e^{-\left(\lambda_{1 a}+\lambda_{1 b}\right)\left(t-t_{0}\right)}\right], \\
& \left.-e^{-\lambda_{2 b}\left(t-t_{0}\right)}\right],
\end{aligned}
$$

and therefore

Counting rate $=\eta_{1}\left(\lambda_{1 a}+\lambda_{1 b}\right) x_{10} e^{-\left(\lambda_{1 a}+\lambda_{1 b}\right)\left(t-t_{0}\right)}+\eta_{2 a} \lambda_{2 a} x_{2 a} 0$

$$
\begin{gathered}
\times e^{-\lambda_{2 a}\left(t-t_{0}\right)}+\eta_{2 b} x_{2 b 0} \lambda_{2 b} e^{-\lambda_{2 b}\left(t-t_{0}\right)}+\frac{\lambda_{1 a} \lambda_{2 a} \eta_{2 a} x_{10}}{\lambda_{2 a}-\lambda_{1 a}-\lambda_{1 b}} \\
\times\left[e^{-\left(\lambda_{1 a}+\lambda_{1 b}\right)\left(t-t_{0}\right)}-e^{-\lambda_{2 a}\left(t-t_{0}\right)}\right]+\frac{\lambda_{1 b} \lambda_{2 b} \eta_{2 b} x_{10}}{\lambda_{2 b}-\lambda_{1 a}-\lambda_{1 b}}\left[e^{-\left(\lambda_{1 a}+\lambda_{1 b}\right)\left(t-t_{0}\right)}\right. \\
\left.-e^{-\lambda_{2 b}\left(t-t_{0}\right)}\right] .
\end{gathered}
$$

The apparent counting rate of the parent species contains a growth factor $g$ which is calculated as before by dividing the observed counting rate of the component with the parent half-life by the counting rate calculated for the pure parent species, to give

$$
\begin{array}{r}
g=1+\left(\frac{\lambda_{1 a}}{\lambda_{1 a}+\lambda_{1 b}}\right)\left(\frac{\lambda_{2 a}}{\lambda_{2 a}-\lambda_{1 a}-\lambda_{1 b}}\right) \frac{\eta_{2 a}}{\eta_{1}}+\left(\frac{\lambda_{1 b}}{\lambda_{1 a}+\lambda_{1 b}}\right) \\
\quad \times\left(\frac{\lambda_{2 b}}{\lambda_{2 b}-\lambda_{1 a}-\lambda_{1 b}}\right) \frac{\eta_{2 b}}{\eta_{1}}
\end{array}
$$

which is analogous to equation II.24. If only one daughter species is active, $\eta_{2 b}$ may be set equal to zero in all the above equations.

Branching decay systems are frequently described in terms of partial half-lives, defined by 


$$
\begin{aligned}
& \mathrm{T}_{\frac{1}{2} \mathrm{a}}=\frac{\ln 2}{\lambda_{1 \mathrm{a}}}=\frac{\lambda_{1 \mathrm{a}}+\lambda_{1 \mathrm{~b}}}{\lambda_{1 \mathrm{a}}} \mathrm{T}_{\frac{1}{2}}, \\
& \mathrm{~T}_{\frac{1}{2} \mathrm{~b}}=\frac{\ln 2}{\lambda_{1 b}}=\frac{\lambda_{1 \mathrm{a}}+\lambda_{1 b}}{\lambda_{1 b}} \mathrm{~T}_{\frac{1}{2}} .
\end{aligned}
$$

\section{Decay Chains With More Than Two Consecutive Decays}

The mathematical techniques applied to two-membered decay chains can readily be extended to multimembered chains, including independent formation and branched decay if necessary. The possible permutations and combinations are so numerous that it is not practical to investigate them in detail; it is enough to recapitulate the mathematical technique used, and to describe another technique which may simplify the labor of deriving the sometimes very elaborate formulae which describe the behavior of such complex systems.

The method of derivation is summarized:

1) Write the differential equation for each member of the chain, as follows: The first time derivative of the amount $x_{i}$ of each species $i$ is equal to the sum of the following three terms:

a) The negative of the product of $x_{i}$ by the total decay constant $\lambda_{i}$ of species $i$.

b) The rate of formation of the species $i$ by any induced nuclear reactions occurring in the system. This expression will in general be the product of a reaction cross section $\sigma_{i}$, a number of atoms $n_{i}$ of target material, and a flux $\phi_{i}$. If more than one reaction is going on, the term for each reaction is written and all such terms are summed. $\phi_{i}$ and $n_{i}$ may be functions of time - for example, the target material may be a species which is itself formed by a nuclear reaction. If $x_{i}$ is being consumed in a nuclear reaction, the term for this reaction is given a negative $\operatorname{sign}$ and $x_{i}$ is used in place of $n_{i}$.

c) The rate of formation of the species i by radioactive decay from one or more parent species. These terms are always given by the product of the number of atoms of the parent 
species present (which is a function of time) by the partial decay constant of that parent species for formation of the species i. Terms from various parents are additive.

2) Start with the equation for the first member of the decay chain. This equation can always be integrated directly, like equation II.1, or by the use of an integrating factor, like equation II.6a, depending on the form of the equation.

3) Substitute the expression obtained into the equations for each member of the second generation.

4) Rearrange the terms in the equations so obtained as in equation II.6b and integrate in the same manner to obtain expressions for the amount of each second-generation species present.

5) Substitute these expressions into the equations for the thirdgeneration species and so on.

6) Multiply the amount of each species by its decay constant and counting efficiency to obtain the counting rate of each species as a function of time. Sum the counting rates where applicable to get the total counting rate of the sample.

7) For each change in the experimental conditions - stopping of an irradiation, purification of a parent species followed by subsequent counting, and the like - use the above technique to give the number of atoms of each species present at the instant of the change; then use this expression as the starting condition for that species and repeat the derivation, using suitably modified differential equations.

The integrands to be integrated will never be more elaborate than simple exponentials unless the system is exposed to a time-dependent flux.

A second approach, which involves somewhat less labor for complex chains, is based on the fact that the amount of any species in a system is always expressible by a constant term (which may be zero) plus a sum of exponential terms involving only the total decay constants of that species and its progenitors in the system, and no others. These decay constants never appear in combinations in the exponents. We can therefore proceed as follows:

1) Write the differential equations of the system, as before. 
2) Express the amount of each species present as a linear combination of the proper exponentials with undetermined coefficients, plus an undetermined constant term.

3) Apply the known starting conditions of the system to give a set of equations relating the unknown coefficients.

4) Substitute the assumed expressions in the differential equations.

5) Obtain additional relations between the coefficients by setting the coefficient of each exponential term in the resulting equations, and the constant term, equal to zero. These relations, together with those obtained in step 3 above, will always be enough to determine the coefficients.

6) Calculate counting rates as before.

An example follows. Let us as sume that species 1 is formed at constant rate in a nuclear reaction; that it decays by branching decay to two species $2 \mathrm{a}$ and $2 \mathrm{~b}$; that each of the se decays to the same active species 3 , which decays to a stable product; and that the starting condition is that the original system contained no active material. For simplicity, we shall measure time from the start of the reaction.

The differential equations are:

$$
\begin{aligned}
& \frac{d x_{1}}{d t}=-\left(\lambda_{1 a}+\lambda_{1 b}\right) x_{1}+\phi n \sigma, \\
& \frac{d x_{2 a}}{d t}=-\lambda_{2 a} x_{2 a}+\lambda_{1 a} x_{1}, \\
& \frac{d x_{2 b}}{d t}=-\lambda_{2 b} x_{2 b}+\lambda_{1 b} x_{1}, \\
& \frac{d x_{3}}{d t}=-\lambda_{3} x_{3}+\lambda_{2 a} x_{2 a}+\lambda_{2 b} x_{2 b} .
\end{aligned}
$$

We assume that

$$
\begin{aligned}
& x_{1}=A_{1}+B_{1} e^{-\left(\lambda_{1 a}+\lambda_{1 b}\right) t}, \\
& x_{2 a}=A_{2 a}+B_{2 a} e^{-\left(\lambda_{1 a}+\lambda_{1 b}\right) t}+C_{2 a} e^{-\lambda_{2 a} t}, \\
& x_{2 b}=A_{2 b}+B_{2 b} e^{-\left(\lambda_{1 a}+\lambda_{1 b}\right) t}+C_{2 b} e^{-\lambda_{2 b} t},
\end{aligned}
$$


$x_{3}=A_{3}+B_{3} e^{-\left(\lambda a^{t \lambda} 1 b^{) t}\right.}+C_{3 a} e^{-\lambda_{2 a} t}+C_{3 b} e^{-\lambda 2 b t}+D_{3} e^{-\lambda_{3} t}$,

and therefore from the starting conditions $(t=0)$

$$
\begin{aligned}
& A_{1}+B_{1}=0, \\
& A_{2 a}+B_{2 a}+C_{2 a}=0, \\
& A_{2 b}+B_{2 b}+C_{2 b}=0, \\
& A_{3}+B_{3}+C_{3 a}+C_{3 b}+D_{3}=0 .
\end{aligned}
$$

Substituting the values of the $\mathrm{x}^{\prime}$ 's from equations II.44a through II.44d into equations II.43a through II.43d, performing the indicated differentiations, and collecting terms, we get

$$
\begin{gathered}
0=-\left(\lambda_{1 a}+\lambda_{1 b}\right) A_{1}+\phi n \sigma \\
0=\left(\lambda_{1 a} A_{1}-\lambda_{2 a} A_{2 a}\right)+\left[\lambda_{1 a} B_{1}-\left(\lambda_{2 a}-\lambda_{1 a}-\lambda_{1 b}\right) B_{2 a}\right] e^{-\left(\lambda_{1 a}+\lambda_{1 b}\right) t} \\
0=\left(\lambda_{1 b} A_{1}-\lambda_{2 b} A_{2 b}\right)+\left[\lambda_{1 b} B_{1}-\left(\lambda_{2 b}-\lambda_{1 a}-\lambda_{1 b}\right) B_{2 b}\right] e^{-\left(\lambda_{1 a}+\lambda_{1 b}\right) t}, \\
0=\left(\lambda_{2 a} A_{2 a}+\lambda_{2 b} A_{2 b}-\lambda_{3} A_{3}\right)+\left[\left(\lambda_{1 a}+\lambda_{1 b}-\lambda_{3}\right) B_{3}+\lambda_{2 a} B_{2 a}+\lambda_{2 b} B_{2 b}\right] \\
\times e^{-\left(\lambda_{1 a}+\lambda_{1 b}\right) t}+\left[\left(\lambda_{2 a}-\lambda_{3}\right) C_{3 a}+\lambda_{2 a} C_{2 a}\right] e^{-\lambda_{2 a} t} \\
+\left[\left(\lambda_{2 b}-\lambda_{3}\right) C_{3 b}+\lambda_{2 b}^{\prime} C_{2 b}\right] e^{-\lambda_{2 b} t}
\end{gathered}
$$

From equation II.46a we get $A_{1}$ and from that and equation II.45a we get $B_{1}$. We then let the coefficients of each exponential term, and the constant term, equal zero separately in equations II.46b and II.46c to give

$$
\begin{aligned}
& \lambda_{1 a} A_{1}-\lambda_{2 a} A_{2 a}=0, \\
& \lambda_{1 a} B_{1}-\left(\lambda_{2 a}-\lambda_{1 a}-\lambda_{1 b}\right) B_{2 a}=0,
\end{aligned}
$$




$$
\begin{aligned}
& \lambda_{1 b} A_{1}-\lambda_{2 b} A_{2 a}=0 \\
& \lambda_{1 b} B_{1}-\left(\lambda_{2 b}-\lambda_{1 a}-\lambda_{1 b}\right) B_{2 b}=0 .
\end{aligned}
$$

Substituting the determined expressions for $A_{1}$ and $B_{1}$ we get expressions for $\mathrm{A}_{2 a}, \mathrm{~B}_{2 a}, \mathrm{~A}_{2 b}$, and $\mathrm{B}_{2 b}$, and by substituting these in equations II.45b and II.45c we get expressions for $\mathrm{C}_{2 a}$ and $\mathrm{C}_{2 b}$. We then let the coefficient of each exponential term in equation II.46d, and the constant term, each equal zero and by substituting in the expressions already determined for the first six coefficients we get expressions for all the rest except $D_{3}$, which is then obtained from equation II.45d. Some labor may often be saved by evaluating only the minimum number of coefficients required to give the desired information - that is to say, the factors making up the observed counting rate of the components being analyzed in the experiment.

It should be emphasized that in any system, whether a simple mixture of species or a complicated tangle of consecutive or branching decays, the decay of the activity of the system can be described by a sum of exponential terms, one component for each species in the system; and that the halflives of the components are identical with the half-lives of the species in the system. Knowledge of the growth and decay formulae derived in this chapter is not necessary for the resolution of such data into components; it is, however, most definitely necessary for the interpretation of the resolved data in terms of measurement of fluxes, cross sections, or other physical properties associated with the experiment.

\section{MATHEMATICAL TECHNIQUES}

\section{Nature of the Problem}

Measurements of radioactivity are, like all physical measurements, subject to both random and systematic error. Unlike many physical measurements, however, measurements of radioactivity are plagued with inherent, unavoidable random errors of quite appreciable size which are due to the very nature of the phenomenon and cannot be eliminated by any refinement of technique. As a result, the elaborate formulae of the preceding chapter must be considered to represent an unattainable ideal. Our initial assumption of precise data must be abandoned. 
We are left with the rather thorny problem of developing an objective technique for using admittedly imperfect data to make a "best" estimate of the quantities of interest in the formulae of Chapter II; of developing a definition of "best" in this sense which is both objective and justifiable; and, further, of forming an estimate of the reliability of the estimates so obtained. In this chapter it has been my objective to collect in one place an assortment of mathematical techniques which apply to the problem. Full development of these disciplines has not been attempted and is, indeed, beyond my competence; I have endeavored to include only those facets of the subject which apply more or less directly to the analysis of data on radioactivity.

\section{Matrix Algebra}

Considerable use will be made of matrix algebra and matrix-vector notation in our subsequent development, since the notation is both concise and elegant.

A matrix is simply a rectangular ordered array of numbers arranged in horizontal rows and vertical columns. The coefficients of a system of simultaneous linear equations, for example, constitute a matrix. If we regard a collection of matrices as a set of mathematical entities, and wish to develop an algebra to handle relationships between the $m$, it is necessary to postulate a set of self-consistent axioms. An algebra can be developed on the basis of any such set; since it is extremely useful to relate the algebra of matrices to the more ordinary algebra of linear equations, our axioms will be derived from those of ordinary algebra through consideration of sets of linear equations.

Let us consider a set of simultaneous linear equations. Such equations consist of statements that the value of a specified linear combination of the members of a set of quantities $x_{i}$ is equal to one of the members another set of quantities, $y_{j}$. If all the $x_{i}$ are known, an unlimited number of $y_{j}$ can be derived by varying the coefficients of the $x_{i}$ in the linear combinations.

Let us consider a set of $m$ linear equations of the form

$$
\sum_{i=1}^{n} a_{j i} x_{i}=y_{j}, \quad 1 \leq j \leq m .
$$


The set of coefficients $a_{j i}$ may be represented by the matrix $A$, where the quantity $a_{j i}$ is the number in the jth row and ith column of $A$. A convenient notation for an element of the matrix $A_{\text {is }} A_{j i}$, the subscripts signifying the row and column, in that order, in which the element is located. We have then for a description of the elements of the matrix $A$

$$
A_{j i}=a_{j i}
$$

We then wish to express the set of $x_{i}$ and the set of $y_{j}$ in matrix form, and define rules of combination of matrices so that the very compact equation

$$
A X=Y
$$

is a valid matrix expression equivalent to the complete set of equations III. 1 for all values of the subscript $j$. We have a choice between expressing the sets of $x_{i}$ and $y_{j}$ as matrices of one row and expressing them as matrices of one column; the choice is arbitrary. In accordance with convention, we shall describe both the set $x_{j}$ and the set $y_{j}$ as matrices of one column; $X$ has as many elements as there are columns of $A$, and $Y$ has as many elements as there are rows of A. A matrix of one column is usually referred to as a vector; we shall define the operation of matrix-vector multiplication as indicated in equation III. 3 by

$$
\sum_{i=1}^{n} A_{j i} X_{i}=Y_{j}
$$

which is obviously identical with equation III.l, except that the quantities concerned are described by their locations in the appropriate matrices $r$ ather than by their magnitudes. The operation is not defined unless the number of elements of $X$ is the same as the number of columns of $A$. In particular, the operation $X X$ is not defined.

The matrix formed from the matrix $A$ by interchanging rows and columns is called the transpose of $A$, symbolized by $A^{t}$. In other words,

$$
A_{i j}^{t}=A_{j i}
$$

The transpose of a vector is a matrix of one row (sometimes called a row vector as opposed to a column vector) and the operation $\mathrm{X}^{\mathrm{t}} \mathrm{X}$ is defined; in fact 


$$
x^{t} x=\sum_{j=1}^{n} x_{j}^{2}
$$

from equation III.4. If $X$ is considered an actual vector in Euclidean $n-s p a c e$ with components of $X_{j}$, the quantity $x^{t} x$ is the square of the amplitude of that vector. The operation $\mathrm{XX}^{\mathrm{t}}$ is also defined; the result is a square matrix whose $i j$ component is $X_{i} X_{j}$.

If in addition to the relationship given by equation III. 3 we have a relationship

$$
\mathrm{BY}=\mathrm{Z} \text {, }
$$

then obviously

$$
Z=B A X \text {. }
$$

We can also derive a relationship between $Z$ and $X$ directly of the form

$$
\mathrm{Z}=\mathrm{CX}
$$

so that for consistency it is desirable to define the operation of multiplication of the matrices $B$ and $A$ in such a manner that

$$
\mathrm{C}=\mathrm{BA}
$$

where the sign of equality implies that each element of the matrix formed by multiplication of the matrices $B$ and $A$ is equal to the corresponding element of the matrix $C$.

It is a simple matter to show that the consistent definition of the operation described in equation III. 10 is, for a matrix $\mathrm{B}$ with $\mathbf{n}$ columns and a matrix $A$ with $n$ rows,

$$
C_{j k}=\sum_{i=1}^{n} B_{j i} A_{i k} \text {. }
$$

The number of columns of B must equal the number of rows of $A$ for the operation to be defined. It can easily be seen that matrix multiplication as defined by equation III. 1 is equivalent to matrix-vector multiplication as defined by equation III.l, considering the columns of the matrix A a s set of vectors each of which is transformed in turn into the corresponding column of the matrix $C$ by multiplication by the matrix $B$. Matrix multiplication is not commutative, that is, $\mathrm{AB}$ does not necessarily equal $\mathrm{BA}$, but can be shown to be associative, i. e. $(A B)(C)=(A)(B C)$. 
Some special forms of matrices are of interest: A square matrix is a matrix with equal numbers of rows and columns; a diagonal matrix is a square matrix whose $i j$ elements are all zero unless $i=j$; an upper triangular matrix is a square matrix whose $i j$ elements are all zero unless $j \geq i$; a lower triangular matrix is a square matrix whose $i j$ elements are all zero unless $i \geq j$; a zero matrix $O$ is a matrix whose elements are all zero; a unit matrix I is a diagonal matrix whose nonzero elements are all unity; and a symmetric matrix is a matrix such that $A_{i j}=A_{j i}$, hence $A=A^{t}$.

The determinant of a square matrix is the determinant whose elements are the elements of that matrix. Its value is, according to the theory of determinants,

$$
|A|=\sum_{j, k}^{m !}\left[(-1)^{n}{\underset{j, k}{m} A_{j k}}^{m}\right]
$$

where within each product of $m$ factors $j$ and $k$ each take on each of the values $l$ to $m$ once and only once, the values of $j$ are taken in order, the sum is taken over all sets of permutations of $k$, and the exponent $n$ is the number of interchanges of the subscripts $k$ necessary to restore them to normal order. If the value of the determinant of a square matrix is zero, the matrix is said to be singular.

It can be shown that a nonsingular square matrix A possesses an inverse $A^{-1}$ such that the product of the two, in either order, is equal to a unit matrix of the same dimensions. In order to evaluate the inverse of a given matrix, we define the cofactor of an element of the matrix $A_{i j}$ to be the determinant of the matrix formed by eliminating the row $i$ and column $j$, multiplied by $(-1)^{i+j}$. The cofactor of $A_{i j}$ is symbolized by $A^{i j}$. It will be recalled, from the theory of determinants, that

$$
|A|=\sum_{i=1}^{m} A_{i j} A^{i j}=\sum_{j=1}^{m} A_{i j} A^{i j} .
$$

In the theory of determinants this is usually spoken of as exparsion by minors. From equation III.13 and the definition of matrix multiplication (equation III.11) it is easy to see that

$$
\left(A^{-1}\right)_{y j}=\frac{A^{j i}}{|A|}
$$


or, that the inverse matrix is formed by replacing each element by the value of its cofactor, dividing each element by the determinant of the entire matrix, and then (note the reversal of indices) transposing the resulting matrix.

From these basic definitions a number of simple relationships become apparent, as follows:

$$
(a A)_{i j}=a A_{i j}
$$

where $a$ is a simple scalar quantity;

$$
\begin{aligned}
& (A+B)_{i j}=A_{i j}+B_{i j}, \\
& A+B=B+A \\
& A(B C)=(A B) C=A B C, \\
& A(B+C)=A B+A C, \\
& (A+B)+C=A+(B+C)=A+B+C .
\end{aligned}
$$

If $A$ is any matrix, $A^{t} A$ is a square symmetric matrix with as many columns as $A$, and $A A^{t}$ is a square symmetric matrix with as many rows as A.

$$
\begin{aligned}
& A I=I A=A, \\
& A \cdot O=O \cdot A=O, \\
& (A B)^{t}=B^{t} A^{t}, \\
& (A B)^{-1}=B^{-1} A^{-1} .
\end{aligned}
$$

We define the term orthogonal as follows: if two vectors $\mathrm{X}$ and $\mathrm{Y}$ have the same number of components, they are orthogonal if

$$
X^{t} Y=Y^{t} X=0 \text {. }
$$

Returning to our set of linear equations, as expressed in equation III.3, if there are $m$ equations of $m$ terms each, and the $m$ components of $Y$ are known while the $m$ components of $X$ are not, we can in principle evaluate the components of $X$ by multiplying both sides of the equation through from the left by $A^{-1}$, if $A$ is not singular, to give

$$
A^{-1} Y=A^{-1} A X=I X=X \text {. }
$$


We shall use this technique in our data analysis; as will be shown shortly, the values of the elements of the inverse matrix will be extremely important to our problem.

\section{Statistical Mathematics}

We make the assumption that the results of an experiment subject to random error, if repeated sufficiently often, will "average out" to the "true" result of an ideal experiment not subject to random errors. Intuitively we feel that most of our actual results will be close to the ideal value, fewer of them somewhat less close, and a very small number quite far away indeed. This leads us to the concept of a distribution function for an experimental measurement subject to random errors; such a measurement will be referred to as a variate. The distribution function for a variate $x$ is a function which describes the fraction of times, in a large number of measurements of $x$, that a measurement of $x$ falls between two specified values. "Fraction of times in a large number of measurements" may be taken as an approximate definition of probability - the rigorous definition may be taken as the limit of that fraction as the number of measurements increases without limit. The utility of the concept stems from the fact that probabilities may often be calculated from first principles.

Distributions can of course arise from other sources than from errors in the measurement of some simple precise quantity. If one measures the weights of a number of supposedly identical objects, some variation will be observed; part of this variation may be due to random er rors in the weighing process and part to actual differences between the objects themselves. In this case the "averaging out" process carried out over a number of measurements refers, not to the "true" weight of any actual object, but to a sort of idealized or typical weight which one would expect such an object to have, a priori.

It should be emphasized that the concept of "average" applies to a distribution; it makes no sense to talk of the "average" of a set of quantities from different distributions unless some correction is applied to reduce these distributions to the same basis. This concept will be discussed in greater detail below. 
The probability of a measurement of the variate $x$ falling in a particular interval is obviously dependent on the size of that interval; as the size of the interval approaches zero, the probability of a measurement falling in that interval becomessimply proportional to the size of the interval.

Let us as sume a coordinate u measured in units of a particular dimensionality - area, for example, or time or pure number. We then define the distribution function $P_{x}(u)$ of the variate $x$, when $x$ is measured in the same units as $u$, to be such that $P_{x}(u) d u$ is the probability that a measured value of $x$ will fall between $u$ and $u+d u$. We will assume that distribution functions are normalized, that is

$$
\int_{-\infty}^{\infty} P_{x}(u) d u=1
$$

This is equivalent to saying that any measurement of $\mathrm{x}$ will result in some finite value.

From the definition, the probability that a measured value of $\mathrm{x}$ will fall between $a$ and $b$ is

$$
P(a \leq x \leq b)=\int_{a}^{b} P_{x}(u) d u
$$

We define the expected value $E[f(x)]$ of any function of $x$ as the limit of the average value from a large number $n$ of measurements, as $n$ increases without limit; therefore

$$
E(x)=\int_{-\infty}^{\infty} u P_{x}(u) d u
$$

and

$$
E[f(x)]=\int_{-\infty}^{\infty} f(u) P_{x}(u) d u
$$

If $f(x)$ is taken as the difference between $x$ and $E(x)$, we get

$$
E[x-E(x)]=\int_{-\infty}^{\infty}[u-E(x)] P_{x}(u) d u=E(x)-E(x)=0
$$

as might be expected; if, however, we consider the expected value of the square of that difference, we are integrating a quantity which is sometimes 
positive and never negative so that we expect a nonzero result. This quantity is called the variance of $\mathbf{x}$ :

$\operatorname{var}(x)=\int_{-\infty}^{\infty}[u-E(x)]^{2} P_{x}(u) d u=E\left\{[x-E(x)]^{2}\right\}=\sigma_{x}^{2}$.

Its square root is the standard deviation of $x, \sigma_{x}$.

It should be noted that the term "variance of $x$ " is somewhat misleading; $\operatorname{var}(x)$ is not a function of $x$, but is a parameter of the distribution function of $x$ along the coordinate $u$. It is a measure of the precision with which $x$ can be measured, or of the "width" of $P_{x}(u)$. Since the dimensions of $\operatorname{var}(x)$ are those of $x^{2}$, while the dimensions of $\sigma_{x}$ are those of $x$, the standard deviation is the quantity which is the easier to interpret in terms of the precision of $x . \quad x$ is often reported as $x \pm \sigma_{x}$. If the form of the distribution function is known, $\pm \sigma_{\mathbf{x}}$ gives information on the size of the interval within which $\mathrm{x}$ is expected to fall; obviously the size of this interval depends on the degree of certainty with which we wish to predict the value of $x$, or the result of a measurement to be made. It is customary to speak of a confidence limit to a certain level; the $95 \%$ confidence limits, for example, define the range in $\mathrm{x}$ within which $95 \%$ of a large number of measurements are expected to fall. (See Fig. III.1.)

The "n percent confidence interval" can be defined explicitly by a such that

$$
\int_{\bar{x}-a}^{\bar{x}+a} P_{x}(u) d u=\frac{n}{100}
$$

which obviously depends on the form of $P_{x}(u)$. It is the confidence interval which determines the significance of a set of results - for example, the question may be asked whether the results of two measurements are believed to be the same with a certain arbitrarily assigned confidence. This is equivalent to considering the difference between the two measurements to be a new variate whose distribution function can be derived, and asking the question whether the value zero lies within the appropriate confidence interval.

If we have two variates $x$ and $y$, we define an analogous quantity, the covariance of $x$ and $y$, by

$$
\operatorname{cov}(x, y)=E\{[x-E(x)][y-E(y)]\}
$$



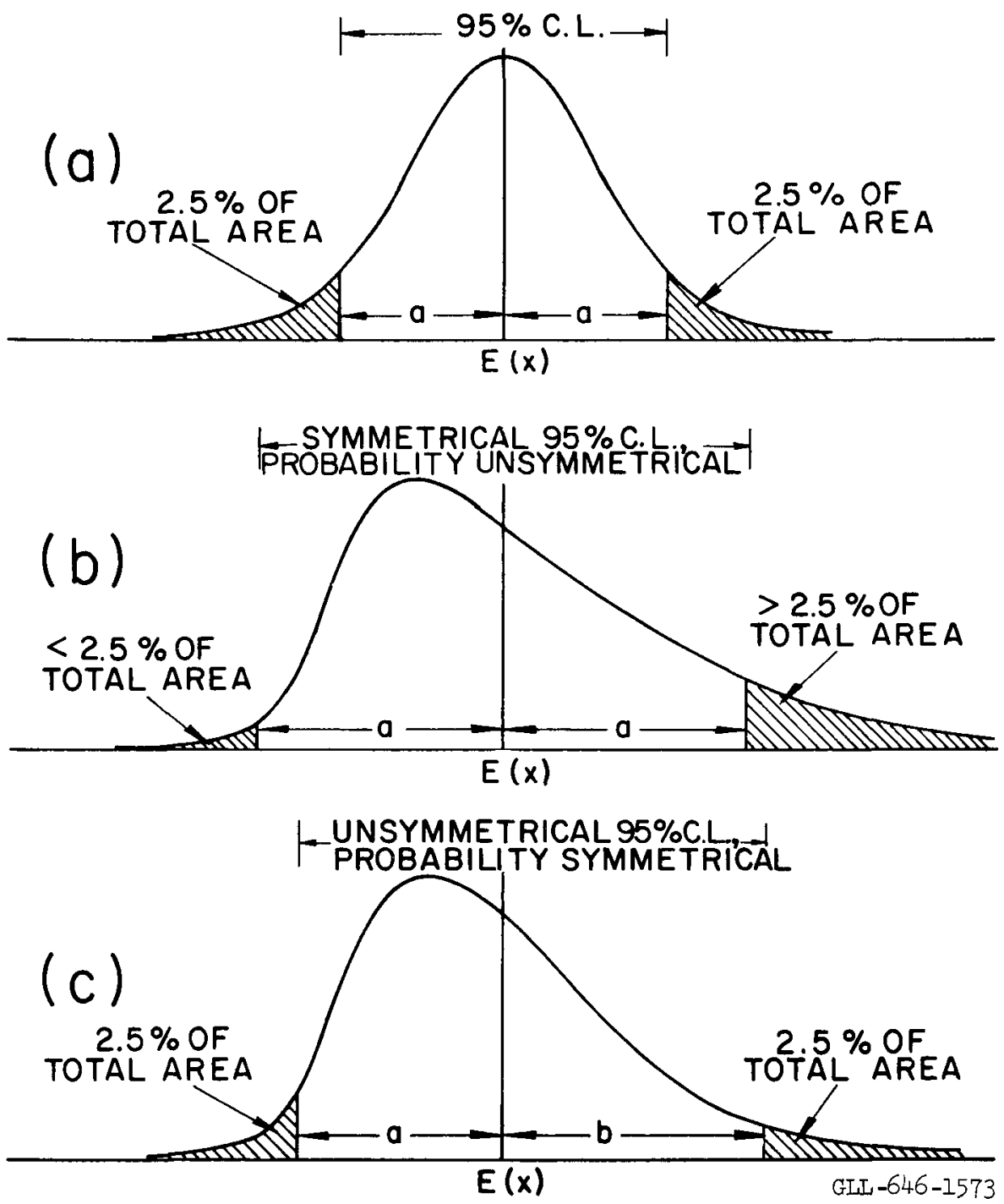

Fig. III.1. Confidence limits: the $95 \%$ confidence limit is shown for three distributions. For a symmetrical distribution (a) a symmetrical interval about $E(x)$ excludes $5 \%$ of the total probability, in two equal parts. This implies that there is a $95 \%$ probability that a single measurement of $x$ will lie between $E(x)+a$ and $E(x)-a$, with an equal probability (of $2.5 \%$ ) that a measurement will lie outside this range on either side. For an asymmetrical distribution one has a choice: (b) shows a symmetrical interval about $E(x)$ such that the probability of a measurement of $x$ falling within this interval is $95 \%$, but a measurement outside this interval is more likely to be too high than too low; (c) shows an asymmetrically defined interval, so placed that a measurement falling outside this interval is as likely to be high as it is to be low. 
Naturally,

$$
\operatorname{var}(x)=\operatorname{cov}(x, x) .
$$

If $\operatorname{cov}(x, y)=0, x$ and $y$ are said to be uncorrelated. $\operatorname{Cov}(x, y)$ is a measure of the correlation between deviations from the expected values of $x$ and $y$, measured simultaneously. If for example one measures both the length and the temperature of each of a set of metal bars, one might not be surprised to find that there was a distribution of both length and temperature; if evaluation of the covariance between length and temperature, however, gives a result which is markedly different from zero, one might then begin to suspect a relationship between the temperature of a bar of metal and its length. One could then get a more realistic idea of the actual distribution of lengths among the metal rods by measuring them all at some constant temperature.

Such a "distribution of lengths" would of course be compounded of the actual distribution in the lengths of the bars and the imprecision of the method of measurement used. If one bar were to be measured a number of times, a distribution would still be obtained, though with a method of measurement sufficiently precise in terms of the unit of measurement such a distribution would be extremely narrow. Information on the precision of the measurement method, obtained by a series of measurements on $a$ single object, can then be used to deduce the actual spread of the distribution in length of a set of bars from the observed total spread. (This deduction will be discussed further under "Analysis of Replicate Samples: Analysis of Variance," Section 7 of Chapter V.)

A number which is not subject to variation is referred to as a consiant. It can easily be seen that, for a constant $a$,

$$
\begin{aligned}
& E(a)=a, \\
& E(a x)=a E(x), \\
& E(a+x)=a+E(x), \\
& \operatorname{var}(a)=0, \\
& \operatorname{cov}(a, x)=\operatorname{cov}(x, a)=0, \\
& \operatorname{var}(a x)=a^{2} \operatorname{var}(x),
\end{aligned}
$$




$$
\begin{aligned}
& \operatorname{var}(a+x)=\operatorname{var} x \\
& \operatorname{cov}(a x, b y)=a b \operatorname{cov}(x, y)
\end{aligned}
$$

$E(x), \operatorname{var}(x)$, and $\operatorname{cov}(x, y)$ are constants of the system.

We are now in a position to develop what we will need of the algebra of expected values, variances, and covariances.

Theorem I: $\underline{E(x+y)=E(x)+E(y)}$.

$$
\begin{aligned}
& E(x+y)=\int_{-\infty}^{\infty} u P_{x+y}(u) d u \\
& P_{x+y}(u)=\int_{-\infty}^{\infty} P_{y}(v) P_{x}(u-v) d v \\
& E(x+y)=\int_{-\infty}^{\infty} \int_{-\infty}^{\infty} u P_{y}(v) P_{x}(u-v) d v d u
\end{aligned}
$$

Reversing the order of integration, and letting $u=u-v+v$, $E(x+y)=\int_{-\infty}^{\infty} P_{y}(v)\left[\int_{-\infty}^{\infty}(u-v) P_{x}(u-v) d u+v \int_{-\infty}^{\infty} P_{x}(u-v) d u\right] d v$

The inner integrals are being taken over the entire range of $u$ with $v$ held constant so that $d u=d(u-v)$ for each value of $v$.

$$
\begin{aligned}
\therefore E(x+y) & =\int_{-\infty}^{\infty} P_{y}(v)\left[\int_{-\infty}^{\infty}(u-v) P_{x}(u-v) d(u-v)\right. \\
& =\int_{-\infty}^{\infty} P_{y}(v)[E(x)+v] d v \\
& \left.=E(x) \int_{-\infty}^{\infty} P_{x}(u-v) d(u-v)\right] d v \\
E(x+y) & =E(x)+E(y) \cdot Q \cdot E \cdot D .
\end{aligned}
$$




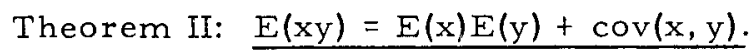

$$
\begin{aligned}
\operatorname{cov}(x, y) & =E\{[x-E(x)][y-E(y)]\} \\
& =E[x y-x E(y)-y E(x)+E(x) E(y)] \\
& =E(x y)-E(y) E(x)-E(x) E(y)+E(x) E(y) \\
& =E(x y)-E(x) E(y) .
\end{aligned}
$$$$
\therefore E(x y)=E(x) E(y)+\operatorname{cov}(x, y) . \quad \text { Q.E. D. }
$$

Corollary: $\underline{E\left(x^{2}\right)=\overline{E(x)}^{2}+\operatorname{var}(x)}$

$$
\begin{aligned}
E\left(x^{2}\right) & =E(x \cdot x)=E(x) E(x)+\operatorname{cov}(x, x) \\
\therefore E\left(x^{2}\right) & =\overline{E(x)}^{2}+\operatorname{var}(x) . \quad \text { Q. E. D. }
\end{aligned}
$$

- Theorem III: $\operatorname{var}(x+y)=\operatorname{var}(x)+\operatorname{var}(y)+2 \operatorname{cov}(x, y)$.

$$
\begin{aligned}
\operatorname{var}(x+y) & =E\left\{[x+y-E(x+y)]^{2}\right\} \\
& =E\left\{[x-E(x)+y-E(y)]^{2}\right\} \\
& =E\left\{[x-E(x)]^{2}+2[x-E(x)][y-E(y)]+[y-E(y)]^{2}\right\} \\
& =E\left\{[x-E(x)]^{2}\right\}+E\left\{[y-E(y)]^{2}\right\}+2 E\{[x-E(x)][y-E(y)]\} .
\end{aligned}
$$

$\operatorname{var}(x+y)=\operatorname{var}(x)+\operatorname{var}(y)+2 \operatorname{cov}(x, y)$. Q.E. D.

Theorem IV: $\operatorname{var}(x-y)=\operatorname{var}(x)+\operatorname{var}(y)-2 \operatorname{cov}(x, y)$.

The proof follows the same method as for Theorem III.

The variance of the product or quotient of two variates depends on more complicated properties of the distribution functions. However, when the standard deviations of the distributions concerned are small compared to their expected values, it is possible to approximate the variance of a product as follows:

Theorem V: $\operatorname{var}(x y) \approx \overline{E(y)}^{2} \operatorname{var}(x)+\overline{E(x)}^{2} \operatorname{var}(y)+2 E(x) E(y) \operatorname{cov}(x, y)$.

$$
\begin{aligned}
\operatorname{var}(x y) & =\operatorname{var}\{[E(x)+x-E(x)][E(y)+y-E(y)]\} \\
& =\operatorname{var}\left\{E(x) E(y)\left[1+\frac{x-E(x)}{E(x)}\right]\left[1+\frac{y-E(y)}{y}\right]\right\} \\
& =E(x)^{2} E(y)^{2} \operatorname{var}\left\{1+\frac{x-E(x)}{E(x)}+\frac{y-E(y)}{E(y)}+\frac{[x-E(x)][y-E(y)]}{E(x) E(y)}\right\}
\end{aligned}
$$

or by equation III. 42 
$\operatorname{var}(x y)=E(x)^{2} E(y)^{2} \operatorname{var}\left\{\frac{x-E(x)}{E(x)}+\frac{y-E(y)}{E(y)}+\frac{[x-E(x)][y-E(y)]}{E(x) E(y)}\right\}$.

Since the distribution is assumed to be narrow, we may neglect the last term to give

$$
\begin{aligned}
\operatorname{var}(x y) & \approx E(x)^{2} E(y)^{2} \operatorname{var}\left[\frac{x-E(x)}{E(x)}+\frac{y-E(y)}{E(y)}\right] \\
& \approx E(x)^{2} E(y)^{2} \operatorname{var}\left[\frac{x}{E(x)}+\frac{y}{E(y)}\right] .
\end{aligned}
$$

Using Theorem III and equation III.43,

$\operatorname{var}(x y) \approx E(x)^{2} E(y)^{2}\left[\frac{1}{E(x)^{2}} \operatorname{var}(x)+\frac{1}{E(y)^{2}} \operatorname{var}(y)+\frac{2}{E(x) E(y)} \operatorname{cov}(x, y)\right]$

$$
\approx E(y)^{2} \operatorname{var}(x)+E(x)^{2} \operatorname{var}(y)+2 E(x) E(y) \operatorname{cov}(x, y) \cdot \quad \text { Q.E. D. }
$$

In the case where the two are independent, then we have

Theorem Va: If the two variates are independent in the statistical sense, then there is an exact relationship:

$$
\frac{\operatorname{var}(x y)=\overline{E(y)}^{2} \operatorname{var}(x)+\overline{E(x)}{ }^{2} \operatorname{var}(y)+\operatorname{var}(x) \cdot \operatorname{var}(y)}{\operatorname{var}(x y)=E\left(x^{2} y^{2}\right)-\overline{E(x y)}^{2}}
$$

Since $x$ and $y$ are independent, $x^{2}$ and $y^{2}$ are independent and consequently

$$
\begin{aligned}
& E\left(x^{2} y^{2}\right)=E\left(x^{2}\right) \cdot E\left(y^{2}\right) \\
& E(x y)=E(x) \cdot E(y) \\
\therefore \operatorname{var}(x y)= & E\left(x^{2}\right) \cdot E\left(y^{2}\right)-\overline{E(x)}^{2} \overline{E(y)}^{2} \\
= & {\left[\overline{E(x)}^{2}+\operatorname{var}(x)\right]\left[\overline{E(y)}^{2}+\operatorname{var}(y)\right]-\overline{E(x)}^{2} \cdot \overline{E(y)}^{2} } \\
= & \overline{E(y)}^{2} \operatorname{var}(x)+\overline{E(x)}^{2} \operatorname{var}(y)+\operatorname{var}(x) \cdot \operatorname{var}(y) .
\end{aligned}
$$

Q.E.D.

Theorem VI: $\operatorname{var}\left(\frac{x}{y}\right) \approx\left[\frac{E(x)}{E(y)}\right]^{2}\left[\frac{\operatorname{var}(x)}{E(x)^{2}}+\frac{\operatorname{var}(y)}{E(y)^{2}}-\frac{2 \operatorname{cov}(x, y)}{E(x) E(y)}\right]$.

$$
\begin{aligned}
\operatorname{var}\left(\frac{x}{y}\right) & =\operatorname{var}\left[\frac{E(x)+x-E(x)}{E(y)+y-E(y)}\right] \\
& =\operatorname{var}\left[\frac{E(x)}{E(y)} \frac{1+\frac{x-E(x)}{E(x)}}{1+\frac{E-E(y)}{E(y)}}\right]
\end{aligned}
$$




$$
\approx\left[\frac{E(x)}{E(y)}\right]^{2} \operatorname{var}\left\{\left[1+\frac{x-E(x)}{E(x)}\right]\left[1-\frac{y-E(y)}{E(y)}\right]\right\}
$$

since we have assumed that $\frac{y-E(y)}{E(y)}$ is small. The proof then follows as for Theorem V

Finally, let us consider a to be a vector with constant components $a_{1}$ and $x$ to be a vector with the same number of varlate components $x_{1}$, then $a^{t} x_{1}$ is a linear combination of the components of $x$. We can define a covariance matrix of $x, K_{x}$, such that

$$
\left(K_{x_{1 j}}\right)=\operatorname{cov}\left(x_{1}, x_{j}\right)
$$

Theorem VII $\operatorname{Var}\left(\mathrm{a}_{\mathrm{x}}\right)-\mathrm{a}^{\mathrm{t}_{\mathrm{K}}} \mathrm{a}$ (in matrix notation)

$$
\begin{aligned}
& a^{t} x=\sum_{1} a_{1} x_{1} \\
& \operatorname{var}\left(\sum_{1} a_{1} x_{1}\right)=E\left\{\left[\sum_{1} a_{1} x_{1}-E\left(\sum_{1} a_{1} x_{1}\right)\right]\right\} \\
& =E\left\{\left[\sum_{1} a_{1} x_{1}-\sum_{1} a_{1} E\left(x_{1}\right)\right]^{2}\right\} \\
& =E\left\{\left[\sum_{j} a_{1}\left(x_{1}-E\left(x_{1}\right)\right)\right]^{2}\right\} \\
& =E\left\{\sum_{I} \sum_{j} a_{1} a_{j}\left[x_{1}-E\left(x_{1}\right)\right]\left[x_{3}-E\left(x_{j}\right)\right]\right\} \\
& =\sum_{1} \sum_{j} a_{1} a_{j} \operatorname{cov}\left(x_{1}, x_{j}\right) \\
& \begin{array}{r}
\sum_{1}\left\{\mathrm{a}_{1} \sum_{\mathrm{J}}\left[\operatorname{cov}\left(\mathrm{x}_{1}, \mathrm{x}_{\mathrm{j}}\right) \mathrm{a}_{\mathrm{J}}\right]\right\}=\mathrm{a}^{\mathrm{t}} \mathrm{K}_{\mathrm{x}} \mathrm{a} \text { in matrıx notation. } \\
\text { Q. E. D. }
\end{array}
\end{aligned}
$$

Theorem VII gives us the means of calculating the variance of any linear combination of variates with constant coefficients if the covariances of all pairs of components are known.

\section{Averaging and Sampling Statistics}

Let us consider first a large number $m$ of independent quantities $x_{1}$ which constitute a distribution. There is no basis for selection among these quantities other than their relation to each other and to the distribution defined by the measurements themselves. The expected value of $\mathrm{x}$ from this distribution 1 g given by 


$$
E(x)=\frac{\sum_{i=1}^{m} x_{i}}{m}
$$

which is a simple arithmetic average, since the "probability of $\mathrm{x}$ having the value $x_{i}$ " 1 s constant for each observed value $x_{i}$. We shall symbolize $E(x)$ by $\mathrm{X}$ for convenience. Also

$$
\operatorname{var}(x)=\frac{\sum_{i=1}^{m}\left(x_{i}-x\right)^{2}}{m}=\sigma_{x}{ }^{2} .
$$

Now let us select a subgroup or sample of $n_{j}$ of the measurements chosen at random, so that $n_{j}<m$. The average of these $n_{j}$ measurements will be, for the ${ }^{\text {th }}$ sample,

$$
\bar{x}_{j}=\frac{\sum_{j}^{n_{j}} x_{i}}{n_{j}}=\sum_{j}^{n_{j}}\left(\frac{1}{n_{j}} x_{i}\right)
$$

(where the summation is taken over the $\mathrm{n}_{j}$ selected values, not necessarily the first $n_{j}$ ). By Theorem III, recalling that the measurements are all independent,

$$
\operatorname{var}\left(\bar{x}_{j}\right)=\sum_{i}^{n_{j}} \operatorname{var}\left(\frac{1}{n_{j}} x_{i}\right),
$$

and by III.4I,

$$
\begin{aligned}
& \operatorname{var}\left(\bar{x}_{j}\right)=\sum_{i}^{n_{j}} \frac{1}{n_{j}^{2}} \operatorname{var}(x)=\frac{1}{n_{j}^{2}} \sum_{i}^{n_{j}} \sigma_{x}^{2}, \\
& \operatorname{var}\left(\bar{x}_{j}\right)=\frac{1}{n_{j}{ }^{2}}\left(n_{j} \sigma_{x}^{2}\right), \\
& \operatorname{var}\left(\bar{x}_{j}\right)=\frac{1}{n_{j}} \sigma_{x}^{2},
\end{aligned}
$$

or, in other words, the variance of the average of a sample of $n_{j}$ measurements is $1 / n_{j}$ the variance of an individual measurement. This result can be used to describe the precision with which the true mean of the total distribution may be estimated from the mean of a random sample drawn from the distribution, provided the variance of the distribution of the sample can be obtained.

*This is strictly true only for an infinitely large population, or if each measurement is replaced in the population after selection so that it may be selected again. 
This variance may be estimated from the distribution of the quantities within the sample, as follows: for a given quantity $x_{i}$ in the $\underline{j}$ th sample

$$
\begin{aligned}
& \left(x_{i}-x\right)=\left(x_{i}-\bar{x}_{j}\right)+\left(\bar{x}_{j}-x\right), \\
& \left(x_{i}-x\right)^{2}=\left(x_{i}-\bar{x}_{j}\right)^{2}+\left(\bar{x}_{j}-x\right)^{2}+2\left(x_{i}-\bar{x}_{j}\right)\left(x_{j}-x\right) .
\end{aligned}
$$

Summing over the total sample,

$$
\sum_{i}^{n_{j}}\left(x_{i}-x\right)^{2}=\sum_{i}^{n_{j}}\left(x_{i}-\bar{x}_{j}\right)^{2}+n_{j}\left(\bar{x}_{j}-x\right)^{2}+2\left(\bar{x}_{j}-x\right) \sum_{i}^{n_{j}}\left(x_{i}-\bar{x}_{j}\right)
$$

but

$$
\sum_{i}^{n_{j}}\left(x_{i}-\bar{x}_{j}\right)=0
$$

\section{Therefore}

$\sum_{i}^{n_{j}}\left(x_{i}-x\right)^{2}=\sum_{i}^{n_{j}}\left(x_{i}-\bar{x}_{j}\right)^{2}+n_{j}\left(\bar{x}_{j}-x\right)^{2}$

$E\left[\sum_{i}^{n_{j}}\left(x_{i}-x\right)^{2}\right]=E\left[\sum_{i}^{n_{j}}\left(x_{i}-\bar{x}_{j}\right)^{2}\right]+n_{j} E\left(x_{j}-x\right)^{2}$,

but

$$
E\left[\sum_{i}^{n_{j}}\left(x_{i}-x\right)^{2}\right]=\sum_{i}^{n_{j}} E\left[\left(x_{i}-x\right)^{2}\right]=n_{j} \sigma^{2}
$$

and

$$
E\left[\left(x_{j}-x\right)^{2}\right]=\operatorname{var}\left(\bar{x}_{j}\right)=\frac{1}{n_{j}} \sigma_{x}^{2}
$$


so that, inserting III. 58 and III. 59 in III.57, and rearranging,

$$
E\left[\sum_{i}^{n_{j}}\left(x_{i}-\bar{x}_{j}\right)^{2}\right]=n_{j} \sigma_{x}^{2}-n_{j}\left(\frac{1}{n_{j}} \sigma_{x}^{2}\right)=\left(n_{j}-1\right) \sigma_{x}^{2} .
$$

Now if we let

$$
s_{j}^{2}=\frac{\sum_{i}^{n}\left(x_{i}-\bar{x}_{j}\right)^{2}}{n_{j}-1},
$$

then

$$
E\left(s_{j}^{2}\right)=\sigma_{x}^{2}
$$

and $s_{j}^{2}$ is an unbiased estimate of $\sigma_{x}^{2}$. Note that when the variance of a total distribution is estimated from a sample of $n_{j}$ quantities, the denominator is $n_{j}-1$; when the variance of the distribution formed by the sample itself is required, the denominator is $n_{j}$.

To recapitulate:

$$
\operatorname{var}(x) \approx \frac{\sum_{i}^{n_{j}}\left(x_{i}-\bar{x}_{j}\right)^{2}}{n_{j}-1}
$$

is an estimated measure of the precision of an individual measurement;

$$
\operatorname{var}(x) \approx \frac{\sum_{i}^{n_{j}}\left(x_{i}-\bar{x}_{j}\right)^{2}}{n_{j}\left(n_{j}-1\right)}
$$

is an estimated measure of the precision of the average of $n_{j}$ measurements.

One can obtain the same result in another manner. Let us as sume that we have a set of $\mathrm{n}$ independent observations $\mathrm{x}_{i}$ belonging to a population with variance $\sigma^{2}$.

Let

$$
u_{i j}=x_{i}-x_{j}
$$

then 


$$
\begin{aligned}
\operatorname{var}\left(u_{i j}\right) & =\operatorname{var}\left(x_{i}\right)+\operatorname{var}\left(x_{j}\right)-2 \operatorname{cov}\left(x_{i}, x_{j}\right)=E\left(u_{i j}^{2}\right)-{\overline{E\left(u_{i j}\right)}}^{2}, \\
\operatorname{var}\left(x_{i}\right) & =\operatorname{var}\left(x_{j}\right)=\sigma^{2}, \\
\operatorname{cov}\left(x_{i}, x_{j}\right) & =\delta_{i j} \sigma^{2} .
\end{aligned}
$$

Now

$$
E\left(u_{i j}\right)=E\left(x_{i}\right)-E\left(x_{j}\right)=0
$$

and therefore

$$
\operatorname{var}\left(u_{i j}\right)=2\left(1-\delta_{i j}\right) \sigma^{2}=E\left(u_{i j}^{2}\right)=E\left(x_{i}^{2}+x_{j}^{2}-2 x_{i} x_{j}\right)
$$

so that an estimate of $\sigma^{2}$ can be made from

$$
\frac{x_{i}^{2}+x_{j}^{2}}{2}-x_{i} x_{j} \approx\left(1-\delta_{i j}\right) \sigma^{2} \text {. }
$$

This estimate can be improved as far as possible from the available data by measuring over all possible pairs.

$$
\sum_{i}^{n} \sum_{j}^{n}\left(\frac{x_{i}^{2}+x_{j}^{2}}{2}-x_{i} x_{j}\right)=n \sum_{i}^{n} x_{i}^{2}-\left(\sum_{i}^{n} x_{i}\right)^{2} \approx \sigma^{2} \sum_{i}^{n} \sum_{j}^{n}\left(1-\delta_{i j}\right),
$$

$\mathrm{n} \sum_{i}^{\mathrm{n}} \mathrm{x}_{i}^{2}-\left(\sum_{i}^{\mathrm{n}} \mathrm{x}_{\mathrm{i}}\right)^{2} \approx\left(\mathrm{n}^{2}-\mathrm{n}\right) \sigma^{2}$

so that

$$
\sigma^{2} \approx \frac{\sum_{i}^{n} x_{i}^{2}-\left(\sum_{i}^{n} x_{i}\right)^{2}}{n} \approx \frac{\sum_{i}^{n}\left(x_{i}-\bar{x}\right)^{2}}{n-1}
$$

is the best estimate of $\sigma^{2}$ available from the data.

The value of this approach lies in the development of the concept of "degrees of freedom" of the system. It can be seen that the term - I in the denominator arises from the restriction that the members of all possible 
pairs are not independent, i. e., that $\operatorname{cov}\left(x_{i}, x_{j}\right)$ is not always zero. It can be shown that in statistical estimation, the denominator in an expression like III. 75 is given by the number of pieces of information (here $n$ ) minus the number of relationships between them. In this case we have one relationship; we are taking an average, so that $\bar{x}$ is expressed in terms of the $x_{i}$. Later we shall see that the resolution of $n$-component systems, when $m$ data points are available, will involve $m$ - $n$ degrees of freedom.

Let us now consider a series of independent measurements of the same quantity, each with its own variance, as sometimes happens. We wish to make a best estimate of the true value of that quantity by averaging the results, but we feel intuitively that the more precise results should be given greater weight in the averaging process; we have also stated that averaging is properly a process which applies only within a given distribution, which is equivalent to saying that the components of the average should all have the same variance. This is not true in the case we are considering; however, since all the measurements purport to be measurements of the same quantity, it should be possible to put them all on the same basis.

We have already seen that measurements which are mean values of sets, or samples, of measurements have variances which are reduced from the variances of individual measurements. Let us consider that a series of $\mathrm{k}$ sample sets have been drawn from a parent universe with a variance $\sigma^{2}$; and let the number of measurements in each set vary from sample to sample. The means of such a series would then represent a series of data such as we are discussing.

In this case, for the jth set, from III.51

$$
\operatorname{var}\left(\bar{x}_{j}\right)=\frac{1}{n_{j}} \sigma^{2} .
$$

Consequently,

$$
n_{j}=\frac{\sigma^{2}}{\operatorname{var}\left(\bar{x}_{j}\right)}
$$

and in order to combine all the observations into one grand average, we take 


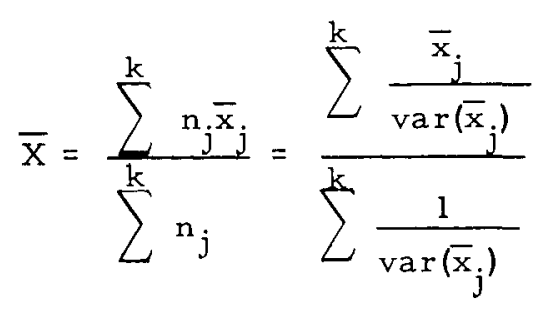

and

$$
\operatorname{var}(\bar{X})=\frac{1}{\sum^{k} n_{j}} \sigma^{2}=\frac{1}{\left[\sum \frac{1}{\operatorname{var}\left(\bar{x}_{j}\right)}\right]}
$$

We therefore see that the proper "weighting factors" for averaging measurements of unlike variances derived from sampling differences are the reciprocals of the variances; it seems apparent that any set of measurements of unlike variances could be duplicated from the same universe by a set with unlike variances arising from sampling, so that the conclusion should be generally applicable.

It can easily be shown that a "weighted average" calculated using the reciprocals of the individual variances for weighting factors is the average having the least variance. If the individual quantities which are to be averaged are not uncorrelated, some complications are encountered, as follows:

Let the set of quantities to be averaged be $X_{j}$, and the weighting factor associated with each $\mathrm{X}_{\mathrm{j}}$ be $\mathrm{W}_{\mathrm{j}}$.

Then

$$
\bar{x}=\frac{\sum_{j} w_{j} x_{j}}{\sum_{j} w_{j}} .
$$

In order to preserve complete independence among the $W_{j}$ we shall not assume that they are normalized. We then have, from III.40,

$$
\operatorname{var}(\bar{X})=\frac{1}{\left(\sum_{j} w_{j}\right)^{2}} \operatorname{var}\left(\sum_{j} w_{j} X_{j}\right)
$$


which from Theorem VII gives us

$$
\operatorname{var}(\bar{x})=\frac{1}{\left(\sum_{j} w_{j}\right)^{2}} \sum_{k} \sum_{j} w_{k} w_{j} \operatorname{cov}\left(x_{k}, x_{j}\right)
$$

We require the set of $w_{j}$ to be chosen to give the best value of $\bar{x}$, which we define to be the value with the least variance. Consequently the derivative of $\operatorname{var}(\bar{X})$ with respect to each of the $W_{j}$, separately, must equal zero. Letting an arbitrary member of the set be $\mathrm{W}_{\ell}$ we have

$$
\begin{aligned}
\frac{\partial}{\partial w_{\ell}}[\operatorname{var}(\bar{X})]=0= & \frac{1}{\left(\sum w_{j}\right)^{4}}\left[\left(\sum_{j} w_{j}\right)^{2} \cdot 2 \sum_{j} w_{j} \operatorname{cov}\left(x_{j}, x_{\ell}\right)\right. \\
& \left.-2\left(\sum_{j} w_{j}\right) \cdot \sum_{j} \sum_{k} w_{j} w_{k} \operatorname{cov}\left(x_{k}, x_{j}\right)\right] .
\end{aligned}
$$

This gives

$$
\sum_{j} w_{j} \operatorname{cov}\left(x_{j}, x_{\ell}\right)=\frac{\sum_{j} \sum_{k} w_{j} w_{k} \operatorname{cov}\left(x_{j}, x_{k}\right)}{\sum_{j} w_{j}} .
$$

The right-hand member of this equation does not depend on $\ell$, the index of the arbitrarily chosen $W_{j}$, and hence may be represented by a constant $C$ whose value is to be determined. We can put equation III.83 in matrixvector form to give

$$
\mathrm{K}_{\mathrm{x}} \mathrm{W}=\mathrm{Ci}
$$

where $C$ is as described above, $W$ is the vector whose components are $W{ }^{\prime}$ $i$ is a vector (of the same dimension as $W$ ) whose components are all unity, and $\mathrm{K}_{\mathbf{x}}$ is the covariance matrix (see Theorem VII) of the $\mathrm{X}_{\mathrm{j}}$. We then have, directly,

$$
\mathrm{W}=\mathrm{CK}_{\mathrm{x}}^{-1}
$$


It is obvious from III.79 that multiplication of the set $W_{j}$ by any arbitrary constant will not change the value of $\bar{X}$, so that $C$ may have any value; it may if desired be chosen to make the $W_{j}$ normalized.

The variance of $\bar{X}$ can be obtained by substituting this value of $W$ into

III.8 1, which gives in matrix notation (realizing that $\left.\sum_{j} w_{j}={ }_{i} t\right)$

$$
\operatorname{var}(\bar{X})=\frac{w^{t} K_{x} W}{C^{2}\left(i^{t} W\right)^{2}}=\frac{C i^{t}\left(K_{x}^{-1}\right) t \cdot K_{x} \cdot C K_{x}^{-1} i}{C^{2}\left(i^{t} K_{x}^{-1} i\right)^{2}} .
$$

Canceling like terms, and letting $\mathrm{K}_{\mathrm{x}} \mathrm{K}_{\mathrm{x}}^{-1}$ equal unity, we have

$$
\operatorname{var}(\bar{X})=\frac{i^{t}\left(K_{x}^{-1}\right)^{t} i}{\left(i^{t} K_{x}^{-1} i\right)^{2}}
$$

but since $\mathrm{K}_{\mathrm{x}}$ is symmetrical, $\mathrm{K}_{\mathrm{X}}^{-\mathrm{l}}$ must be symmetrical, and hence

$$
\operatorname{var}(\bar{X})=\frac{1}{i \mathrm{t}_{x}^{-]} i}
$$

In matrix notation,

$$
\bar{X}=\frac{W^{t} X}{i^{t} W}=\frac{i^{t} K_{x}^{-1} x}{i^{t} K_{x}^{-1} i}
$$

In the simple case where the $x_{j}$ are in fact uncorrelated, $K_{x}$ is a diagonal matrix whose diagonal elements are the variances of the $X_{j}$; the diagonal elements of the inverse of $\mathrm{K}_{\mathrm{x}}$ are therefore the reciprocals of the variances of the $X_{j}$. Inserting these values into III. 88 and III.89, we arrive at III. 77 and III.78.

\section{The Least-Squares Solution}

If we are given a set of data, knowing that the measurements of the set are subject to random error, and if we are given an equation of known mathematical form containing a number of unknown parameters whose values are to be determined by fitting the equation to the data, what is the procedure which will give the "best" estimates of the parameters from the admittedly 
imperfect data? We obviously wish to minimize the average deviation between the observed points and the calculated points in some manner. The average of the algebraic values of the deviations will not do, since a large negative deviation would counterbalance a large positive one; we might consider minimizing the average of the absolute values of the deviations. A better approach is to minimize the average of the squares of the deviations of the observed from the calculated points. We shall show that this approach leads to expected values of the parameters which are the true values. In practice it is enough to minimize the sum of the squares of the deviations, properly weighted.

We must first consider the question of the relative worth of the experimental points. If we have no information about the estimated precision of each point, we have no choice but to treat them all alike; furthermore, if we have reason to believe that the points are all equally precise, we have every reason to treat them alike. However, if we have an estimate of the precision of each point, and the estimates are not all equal, we shall see that the points should be weighted.

We will see that the appropriate weighting factors in this case are inversely proportional to the variances of the points. Let us assume that we have a set of $m$ measurements $y_{j}$ of known variance; and also that each point is to be fitted by an expression which is a linear combination of $\mathrm{n}$ known functions or quantities $a_{i j}$ with unknown coefficients $x_{i}$ which are constant over the total set of measurements. We know that if the equations are consistent (as they must be to represent a real case) and if $m \geq n$, solutions for the $x_{i}$ are possible; let us assume $m>n$. We then have a set of $m$ equations

$$
y_{j}=\sum_{i=1}^{n} a_{i j} x_{i}
$$

In general any well-selected set of parameters $x_{i}$ will yield a calculated set of values of $y_{j}$ which differ from the observed values by a set of small quantities,

$$
E_{j}=\sum_{i=1}^{n} a_{i j} x_{i}-y_{j}
$$


We wish to minimize $S$, the weighted sum of the squares of the $E_{j}$, with respect to each parameter $x_{k}$. Let us for the moment assume that the appropriate weighting factor is proportional to the reciprocal of $\operatorname{var}\left(\mathrm{y}_{\mathrm{j}}\right)$. Then

$$
\begin{aligned}
& S=\sum_{j=1}^{m}\left[\frac{\left.\left(\sum_{i=1}^{n} a_{i j} x_{i}-y_{j}\right)^{2}\right]}{\operatorname{var}\left(y_{j}\right)}\right] \\
& \frac{\partial S}{\partial x_{k}}=\sum_{j=1}^{m} \frac{2 a_{k j}\left(\sum_{i=1}^{n} a_{i j} x_{i}-y_{j}\right)}{v a r\left(y_{j}\right)}=0, \\
& \sum_{j=1}^{m} \sum_{i=1}^{n}\left(a_{k j} a_{i j} x_{i}\right)-a_{k j} y_{j} \\
& \operatorname{var}\left(y_{j}\right)
\end{aligned}
$$

or, reversing the order of summation,

$$
\sum_{i=1}^{n}\left\{\left[\sum_{j=1}^{m} \frac{a_{k j} a_{i j}}{\operatorname{var}\left(y_{j}\right)}\right] x_{i}\right\}=\sum_{j=1}^{m} \frac{a_{k j} y_{j}}{\operatorname{var}\left(y_{j}\right)}
$$

for each value of $k$ from 1 to $n$. These equations are referred to as the normal equations of the system. Their solutions are the values for the $x_{i}$ which minimize the quantity $S$.

It can be seen that if the equations of the original set III. 90 are weighted by the reciprocal of the square root of the variance of $y_{j}$, the normal equations can be put in matrix notation very simply. Let the matrix $A$, the vector $X$, and the vector $Y$ be defined by

$$
A_{i j}=\frac{a_{i j}}{\left[\operatorname{var}\left(y_{j}\right)\right]^{1 / 2}}
$$




$$
\begin{aligned}
& x_{1}=x_{1} \\
& Y_{j}=\frac{y_{j}}{\left[\operatorname{var}\left(y_{j}\right)\right]^{1 / 2}}
\end{aligned}
$$

Then the equation III. 90 can be written

$$
\mathrm{AX}=\mathrm{Y} \text {. }
$$

Note that $A$ is not a square matrix since $m>n$. If we multiply both sides of the equation from the left by the transpose of the matrix $A$, we get

$$
A^{t} A X=A^{t} Y,
$$

which by the rules of matrix algebra can be shown to be precisely equivalent to equation III $92 \mathrm{~d}$, including the proper weighting factor. The solution in matrix notation is then

$$
X=\left(A^{t} A\right)^{-1} \cdot A^{t} Y
$$

For the remaining discussion we shall assume that the equations are properly weighted at the start.

Kenney and Keeping ${ }^{2}$ present the following demonstration that if the equations are so weighted the expected values of the $x_{1}$ calculated from the normal equations III. 95 are the true values of the system.

Let us suppose that the $y_{j}$ of equation III.90 are distributed about their true values $\eta_{1}$ with a common variance $\sigma^{2}$ (which will be true if the equations are properly weighted) and that they are independent. Let us write the normal equations III. 95 in the form

$$
\mathrm{BX}=\mathrm{g}
$$

where the matrix $B$ is equal to $A^{t} A$ and the vector $g$ is equal to $A^{t} Y$. Then

$$
\begin{aligned}
X & =B^{-1} g, \\
x_{j} & =\sum_{k}\left(B^{-1}\right)_{j k} g_{k}, \\
\therefore E\left(x_{j}\right) & =\sum_{k}\left(B^{-1}\right)_{j k} E\left(g_{k}\right) .
\end{aligned}
$$

J. F. Kenney and E. S. Keeping, The Mathematics of Statistics, Part Two (D. Van Nostrand Co., Inc., Princeton, 1951), 2nd Ed., P. $309 \mathrm{ff}$. 
We obtain the expected value of $g_{k}$ from the expected values of the $y_{j}$,

$$
E\left(g_{k}\right)=\sum_{1} a_{k 1} E\left(y_{1}\right),
$$

and the expected values of the $y_{1}$ from the true values $\xi_{\ell}$ of the $x_{\ell}$,

$$
E\left(y_{1}\right)=\sum_{\ell} a_{\ell 1} \xi_{\ell} .
$$

Substituting back into equation III.98c,

$$
E\left(x_{j}\right)=\sum_{k} \sum_{1} \sum_{\ell}\left(B^{-1}\right)_{j k} \cdot a_{k_{1}} \xi_{\ell} \cdot a_{\ell 1} \cdot
$$

Rearranging the order of summation,

$$
E\left(x_{j}\right)=\sum_{\ell}\left\{\xi_{\ell} \sum_{\mathrm{k}}\left[\left(\mathrm{B}^{-1}\right)_{\mathrm{jk}} \sum_{1} \mathrm{a}_{\mathrm{k} 1} \mathrm{a}_{\ell 1}\right]\right\}
$$

but

$$
\begin{aligned}
& \sum_{1} a_{k_{1}} a_{\ell 1}=B_{k \ell^{\prime}} \\
& \therefore E\left(x_{\jmath}\right)=\sum_{\ell}\left[\xi_{\ell} \sum_{k}\left(B^{-1}\right)_{j k} \cdot B_{k \ell}\right] .
\end{aligned}
$$

Now by the definition of an inverse matrix and the laws of matrix multiplication,

$$
\begin{aligned}
& \sum_{\mathrm{k}}\left(\mathrm{B}^{-1}\right)_{\mathrm{jk}} \mathrm{B}_{\mathrm{k} \ell}=\delta_{\mathrm{j} \ell}=\left\{\begin{array}{l}
1, j=\ell, \\
0, j \neq \ell .
\end{array}\right. \\
& \therefore E(x)=\sum_{l} \xi_{l} \delta_{\jmath \ell}-\xi_{j} \text {. Q.E.D. }
\end{aligned}
$$

That is to say, the expected values of the $x_{j}$ resulting from solution of the properly weighted normal equations are the true values for the system. 
We also wish information concerning the variances of the $x_{j}$ so obtained, and of the variance of any calculated $y$ using a suitable set of $a_{1}$. For both these problems we need the covariance matrix $K_{x}$ of the vector $x$ (see the definition, equation III.4l).

We shall show, following Scheffe, ${ }^{3}$ that the inverse of the matrix of the normal equation, multiplied by the common variance of the $y$, is the covariance matrix of $x$.

Let us define a vector $\mathrm{V}$ whose components are jointly distributed random variates all having finite variance. Let us further define the expected value of a matrix or vector to be the matrix or vector formed from the expected values of its components. Then we can write

$$
K_{V}=E\left\{[V-E(V)][V-E(V)]^{t}\right\} \text {. }
$$

Now if we have a vector $W$ with variate components, and a matrix $A$ with constant components, and if

$$
\mathrm{W}=\mathrm{AV},
$$

then

$$
\begin{aligned}
& K_{W}=E\left\{[W-E(W)][W-E(W)]^{t}\right\}, \\
& K_{W}=E\left\{A[V-E(V)][V-E(V)]^{t} A^{t}\right\}, \\
& K_{W}=A E\left\{[V-E(V)][V-E(V)]^{t}\right\} A^{t},
\end{aligned}
$$

or

$$
K_{W}=A K_{V} A^{t}
$$

The normal equations are written,

$$
A^{t} A X=A^{t} Y
$$

and we assume that the covariance matrix of $Y$ is

$$
K_{y}=\sigma^{2} I
$$

since the $y_{j}$ are all independent and have a common variance $\sigma^{2}$.

\footnotetext{
${ }^{3}$ H. Scheffé, The Analysis of Variance (John Wiley \& Sons, Inc., New York 1959), pp 8-12.
} 


$$
X=\left(A^{t} A\right)^{-1} A^{t} Y
$$

and from III. 102

$$
\begin{aligned}
K_{x} & =\left(A^{t} A\right)^{-1} A^{t} \cdot K_{y} \cdot\left[\left(A^{t} A\right)^{-1} A^{t}\right]^{t} . \\
\therefore K_{x} & =\sigma^{2}\left(A^{t} A\right)^{-1} \cdot A^{t} \cdot I \cdot A \cdot\left[\left(A^{t} A\right)^{-1}\right] t
\end{aligned}
$$

But since $A^{t} A$ is symmetrical, $\left(A^{t} A\right)^{-1}$ is symmetrical and

$$
\begin{aligned}
{\left[\left(A^{t} A\right)^{-1}\right]^{t} } & =\left(A^{t} A\right)^{-1} \cdot \\
\therefore K_{x} & =\sigma^{2}\left(A^{t} A\right)^{-1} \cdot A^{t} A \cdot\left(A^{t} A\right)^{-1} . \\
\therefore K_{x} & =\sigma^{2}\left(A^{t} A\right)^{-1} \cdot \text { Q. E. D. }
\end{aligned}
$$

We now need an unbiased estimate of $\sigma^{2}$. We define the residual $\mathrm{p}_{\mathrm{j}}$ by

$$
P_{j}=y_{j}-Y_{j}
$$

where $y_{j}$ is the (weighted) observed value of $y$ and $Y_{j}$ is the (weighted) value computed from the least-squares solution values of the parameters $x$. We shall show that, if we have $n$ parameters and $m$ points,

$$
\sigma^{2}=\frac{E\left(\sum_{j=1}^{m} p_{j}^{2}\right)}{m-n}
$$

following the treatment of Kenney and Keeping. ${ }^{4}$ We define the error $\triangle_{j}$ by

$$
\Delta_{j}=y_{j}-\eta_{j}
$$

where $\eta_{j}$ is the true value of $y_{j}$.

We have

$$
E\left(y_{j}^{2}\right)=E\left[\left(\eta_{j}+\Delta\right)^{2}\right]=\eta_{j}^{2}+\sigma^{2}
$$

since $E\left(\triangle_{j}\right)$ is zero. If the true values of the $x_{i}$ are $\xi_{i}$, as before,

$$
\eta_{j}=\sum_{i} \xi_{i} a_{i j}
$$




$$
\therefore E\left(y_{j}^{2}\right)=\sum_{i=1}^{n} \sum_{k=1}^{n} \xi_{i} \xi_{k} a_{i j} a_{k j}+\sigma^{2}
$$

Summing over all j,

$\sum_{j=1}^{m} E\left(y_{j}^{2}\right)=E\left(\sum_{j=1}^{m} y_{j}^{2}\right)=m \sigma^{2}+\sum_{i=1}^{n} \sum_{k=1}^{n} \xi_{i} \xi_{k}\left(\sum_{j=1}^{m} a_{i j} a_{k j}\right)$,

$\sum_{j=1}^{m} a_{i j} a_{k j}=B_{i k}$ where $B=A^{t} A$.

$\therefore E\left(\sum_{j=1}^{m} y_{j}^{2}\right)=m \sigma^{2}+\sum_{i=1}^{n} \sum_{k=1}^{n} \xi_{i} \xi_{k} B_{i k}$

We write the normal equations as

$$
B X=A^{t} A X=A^{t} y=G \text {, }
$$

$$
G_{i}=\sum_{k=1}^{n} B_{i k} x_{k}
$$

$$
\sum_{i=1}^{n} x_{i} G_{i}=\sum_{i=1}^{n} \sum_{k=1}^{n} B_{i k} x_{i} x_{k}
$$

$E\left(\sum_{i=1}^{n} X_{i} G_{i}\right)=\sum_{i=1}^{n} \sum_{k=1}^{n} B_{i k} E\left(x_{i} x_{k}\right)=\sum_{i=1}^{n} \sum_{k=1}^{n} B_{i k}\left[\xi_{i} \xi_{k}+\operatorname{cov}\left(x_{i} x_{k}\right)\right]$,

$E\left(\sum_{i=1}^{n} x_{i} G_{i}\right)=\sum_{i=1}^{n} \sum_{k=1}^{n} B_{i k}\left[\xi_{i} \xi_{k}+\sigma^{2}\left(B^{-1}\right){ }_{i k}\right]$

$E\left(\sum_{i=1}^{n} X_{i} G_{i}\right)=\sum_{i=1}^{n} \sum_{k=1}^{n} B_{i k} \xi_{i} \xi_{k}+n \sigma^{2}$,

since $\sum_{k=1}^{n} B_{i k}\left(B^{-1}\right)_{i k}=\sum_{k=1}^{n} \delta_{i k}=1$. 


$$
\sum_{i=1}^{n} \sum_{k=1}^{n} \xi_{i} \xi_{k} B_{i k}=E\left(\sum_{i=1}^{n} x_{i} G_{i}\right)-n \sigma^{2} .
$$

Now substituting in III. 85 and rearranging,

$$
E\left(\sum_{j=1}^{m} y_{j}^{2}\right)-E\left(\sum_{j=1}^{m} x_{j} G_{j}\right)=(m-n) \sigma^{2} .
$$

We have also

$$
\begin{aligned}
& p=y-Y=y-A X \\
& A^{t} p=A^{t} y-A^{t} A X=G-A^{t} A X=0 \\
& \sum_{j=1}^{m} p_{j}^{2}=p^{t} p=\left(y^{t}-Y^{t}\right) p=y^{t} p-Y^{t} p \\
& Y^{t} p=X^{t} A^{t} p=X^{t} \cdot 0=0 \\
& \therefore \sum_{j=1}^{m} p_{j}^{2}=y^{t} p=y^{t}(y-A X)=y^{t} y-G^{t} X . \\
& \therefore \sum_{j=1}^{m} p_{j}^{2}=\sum_{j=1}^{m} y_{j}^{2}-\sum_{j=1}^{m} X_{j} G_{j} \cdot \\
& E\left(\sum_{j=1}^{m} p_{j}^{2}\right)=E\left(\sum_{j=1}^{m} y_{j}^{2}\right)-E\left(\sum_{j=1}^{m} X_{j} G_{j}\right) .
\end{aligned}
$$

Substituting in equation III. 128, and rearranging terms,

$$
\sigma^{2}=\frac{E\left(\sum_{i} p_{j}^{2}\right)}{n-n}
$$

so that

$$
s^{2}=\frac{\sum_{j} p_{j}^{2}}{m-n}
$$


is an unbiased estimate of $\sigma^{2}$ It should be borne in mind that $S^{2}$ is an estimate which corrects for variances which were not included in the weighting, consequently a result for $S^{2}$ of less than unity means either that, fortuitously, the data fit the calculated curve better than one may reasonably expect, or, alternatıvely, that the original error estımates were too large. In the first case one should not insert the calculated value of $S^{2}$ into equation III $104 \mathrm{f}$, but should merely assume $\sigma^{2}$ to be unity, if however there is any reason to believe that the orıginal error estimates were made by a non-objective method, the result obtained is essentially meaningless One object of this monograph is to provide experimenters with a set of rules for making the best estimate of the errors actually involved in their measurements, if a subjective, conservative "guess" is used, not only is the resulting final estimate of the precision of the experiment meaningless, but useful information is sometimes lost The common procedure of guessing the precision of experimental data is indefensible

If one now wishes to know the precision of each of the parameters $x_{1}$ in our least-squares solution, it can readily be obtained from the inverse matrix of the normal equations, since

$$
x_{1}=x_{1} \pm \sqrt{S^{2}\left[\left(A^{t} A\right)^{-1}\right]_{11}}
$$

A derıvation exists (due to $H$ Henris and $H \quad B$ Levy of this laboratory) to show that the choice of weighting factors described by Eqs. III 93a, b and c is in fact that cholce which results in minimum values for the components of the matrix $K_{X}$, and hence in the most precise estimate of the components of the vector $X$ The derivation follows

We have the relationship

$$
A_{0} X_{0}=Y_{0}
$$

where $A_{0}, X_{0}$, and $Y_{0}$ represent matrices of "true" values Suppose we have $m$ observed components of $Y$ and there are $n$ unknown components of $X$, with $m>n$, and that the components of $Y$ are subject to error

We wish to estimate $\theta$, which is a linear function of the $x_{1}$ 's

$$
\theta=c^{t} X
$$

where $c^{I}$ is a given row vector We wish to ask

How can we estimate $\mathrm{x}$ so that our resulting estimate of $\theta$ will have minimum varıance?

$$
\operatorname{var} \theta=c^{t} K_{x} c \quad \text { (see Theorem VII, page (39)) }
$$

where $K$ is the covariance matrix for the estimate of $x$ 
Let $A$ and $Y$ represent matrices of observed values

A is $(m \times n) \quad Y$ is $(m \times 1)$

$$
\begin{array}{ll}
A-A_{0}+d A & E(d A)-0 \\
Y=Y_{0}+d Y & E(d y)=0
\end{array}
$$

Let us defıne our estımate of $\mathrm{X}$ as

$$
\mathrm{X}-\mathrm{PY} \text { where } \mathrm{PA}=\mathrm{I} \text { defines } \mathrm{P}
$$

We need

$$
\begin{aligned}
K_{X} & =E\left\{\left(X-X_{0}\right)\left(X-X_{0}\right)^{t}\right\} \\
A_{0} X_{0} & =Y_{0} \\
{[A-(d A)] X_{0} } & =Y-(d Y) \\
A_{0} & =Y-[(d Y)-(d A) X]
\end{aligned}
$$

since

$$
\begin{aligned}
P A & =I \\
X_{0} & =P Y-P\left[(d Y)-(d A) X_{0}\right] \\
X-X_{0} & =P\left[(d Y)-(d A) X_{0}\right] \\
K_{X} & =E\left\{\left(X-X_{0}\right)\left(X-X_{0}\right)^{1}\right\}=E\left\{P[(d Y)-(d A) X][(d Y)-(d A) X]^{t} P^{t}\right\} \\
K_{X} & -P E\left\{[(d Y)-(d A) X]\left[(d Y)-(d A) X_{0}\right]^{t}\right\} P^{t}
\end{aligned}
$$

Let

$$
V \equiv E\left\{\left[(d Y)-(d A) X_{0}\right]\left[(d Y)-(d A) X_{0}\right]\right\}
$$

$\mathrm{V}$ is an $\mathrm{m} \times \mathrm{m}$ symmetric, positive-semidefinite matrix, that is $\mathrm{a}^{\mathrm{t}} \mathrm{Va} \geq 0$

$$
\mathrm{K}_{\mathrm{x}}=\mathrm{PVP}^{\mathrm{t}}
$$

Since

$$
\mathrm{P} \underset{\mathrm{m} \times \mathrm{n}}{\mathrm{A}}=\underset{\mathrm{n} \times \mathrm{n}}{\mathrm{I}}
$$

$\mathrm{P}$ must be a $\mathrm{n} \times \mathrm{m}$ matrix that can be written in the form (TA) ${ }^{-1} \mathrm{~T}$ where $\mathrm{T}$ is an $n \times m$ matrıx such that the product $T A$ is a non-singular matrix.

Therefore $\mathrm{T}$ must be of rank $\mathrm{n}$ (A must also be of rank $\mathrm{n}$ ). We can choose $\mathrm{T}$ so that $\operatorname{var} \theta$ is a minimum. 
Let $t_{1 \mathrm{~J}}$ represent the elements of the $\mathrm{T}$ matrix Then for var $\theta$ to be $\mathrm{a}$ minimum

$$
\frac{\partial(\operatorname{var} \theta)}{\partial t_{1 j}}=0 \text { for all } t_{1 j}
$$

Since

$$
\operatorname{var} \theta=c^{t} K_{x} c
$$

then

$$
\frac{\partial(\operatorname{var} \theta)}{\partial t_{1 j}} w_{1} l 1=0 \text { only if } \quad \frac{\partial K_{x}}{\partial t_{1 j}}=0,
$$

since $K_{X}$ is square, symmetric, and positıve-semidefinite. Since

$$
\begin{aligned}
& K_{x}=P V P^{t} \\
& \frac{\partial K_{x}}{\partial t_{1 j}}=\frac{\partial P}{\partial t_{1 j}} V P^{t}+P V \frac{\partial P^{t}}{\partial t_{1 J}}=0 .
\end{aligned}
$$

Since V is symmetric, positive-semidefinite, both

$$
\frac{\partial P}{\partial t_{1 J}} V P^{t} \text { and } P V \frac{\partial P^{t}}{\partial t_{1 j}} \text { must equal } 0 .
$$

Consider

$$
\begin{aligned}
\frac{\partial P}{\partial t_{1 J}} V P^{t} & =0 \\
P & =(T A)^{-1} T \\
\frac{\partial P}{\partial t_{1 j}} & =(T A)^{-1}\left[\frac{\partial T}{\partial t_{1 j}}-\frac{\partial T}{\partial t_{1 j}} A(T A)^{-1} T\right] \\
\frac{\partial P}{\partial t_{1 j}} V P^{t} & =(T A)^{-1}\left[\frac{\partial}{\partial t_{1 j}}-\frac{\partial T}{\partial t_{1 J}} A(T A)^{-1} T\right] V T^{t}\left(A^{t} T^{t}\right)^{-1}=0
\end{aligned}
$$

Therefore

$$
\frac{\partial T}{\partial t_{1 j}}\left[V T^{t}-A(T A)^{-1} T V T^{t}\right]=0 \text {. }
$$

In order for the above to be $=0$ for all $t_{1 \mathrm{j}}$ it is necessary that $V T^{t}-A(T A)^{-1} \mathrm{TVT}^{\mathrm{t}}=0$ Therefore,

$$
A(T A)^{-1} T\left(V T^{t}\right)=\left(V T^{t}\right) \text {. }
$$


Let

$$
\begin{array}{ll}
M-A(T A)^{-1} T & \text { an } m \times m \text { matrix of rank } n . \\
B=V_{T}^{t} & \text { an } m \times n \text { matrix }
\end{array}
$$

Consider the matrix MI

M is an idempotent matrix of order $m$ and rank $n$ It will have $n$ characteristic roots $\lambda=1$ and $m-n$ characteristic $\operatorname{roots} \lambda=0$. Therefore, there will be no more than $\mathrm{n}$ independent vectors $\mathrm{z}$ for which

$$
\mathrm{Mz}=\mathrm{z} \text {. }
$$

We know

$$
\mathrm{MB}=\mathrm{H}
$$

so that the $\mathrm{n}$ column vectors of $\mathrm{B}$ must constıtute such a set of $\mathrm{n}$ independent elgenvectors associated with $\mathrm{MI}(\mathrm{l}=1)$.

But also

$$
\mathrm{MA}=\mathrm{A} \text {. }
$$

Therefore the column vectors of A must also constitute such a set Since there can only be $\mathrm{n}$ independent eigenvectors associated with $\lambda=1$, the column vectors of $B$ must be linear combinations of the column vectors of $A$ and

$$
\begin{aligned}
& B= A Q \quad \text { where } Q \text { is an elementary matrix } \\
& B= V T^{t}-A Q \\
& T^{t}=V^{-1} A Q \\
& T=Q^{t} A^{t} V^{-1} \\
& P=(T A)^{-1} T \\
& P=\left(Q^{t} A^{t} V^{-1} A\right)^{-1} Q^{t} A^{t} V^{-1} \\
& P=\left(A^{t} V^{-1} A\right)^{-1}\left(Q^{t}\right)^{-1} Q^{t} A^{t} V^{-1} \\
& P=\left(A V^{1} A\right)^{-1} A^{1} V^{-1}
\end{aligned}
$$

This is identical to a weighted least squares method with the weighting matrix proportional to $\mathrm{V}^{-1}$.

Note that the chore of $Q$ has no effect on $P$ so that the estrmate of $x$ and its variance is not dependent on $Q$. 
The quantity $x^{2}$ may be defined in terms of the unweighted residuals $r_{j}$ by

$$
x^{2}=\sum_{j=1}^{m} \frac{r^{2}}{\operatorname{var}\left(y_{j}\right)} .
$$

If our weighting factors for the least-squares solution are chosen to correspond with the true values of the variances of the $y_{j}$, then obvously the value of $\sigma^{2}$ is one, and from III. 130 we get

$$
\mathrm{E}\left(\chi^{2}\right)=\mathrm{m}-\mathrm{n} \text {. }
$$

It can be shown that

$$
\operatorname{var}\left(x^{2}\right)=2(m-n)
$$

and tables exist ${ }^{5,6}$ showing the probability of occurrence through pure random error of any deviation $x^{2}-E\left(x^{2}\right)$, measured in terms of $\left[\operatorname{var}\left(x^{2}\right)\right]^{1 / 2}$. The calculated value of $\chi^{2}$ may then be used as a test of the goodness of $f_{1} t$ of the assumed equations to the data. If the calculated value of $\chi^{2}$ exceeds the expected value by an amount too great to attribute to random error, one suspects at least one of three difficulties. first, an error in the calculation, second, an improper estimate of the varıances of the $y_{j}$, or third, an improper form for the function being fitted to the data If the first source of trouble is eliminated through careful checking, a cholce can frequently be made between the other two on the following basis. if the known functions $a_{1 j}$ of the equation which is being fitted to the data are themselves smoothly varying functions of a precisely known parameter such as the time of the observation, then the residuals should be tabulated in the same order as the corresponding values of that parameter If any systematic trend then exists in the residuals, the form of the equation is probably incorrect, if the residuals are scattered at random, the estimates of variance used for the weighting factors are too small.

In many cases, and specifically in the analysis of radioactive decay data, the number of terms in the equation is sometimes uncertain. It is desirable to determine whether the presence of a particular term or set of terms causes a significant improvement in the fit of the equation to the data. A test for this

${ }^{5}$ R. A. Fisher and F. Yates, Statistical Tables (Hafner, New York, 1948).

${ }^{6}$ E. S. Pearson and H. O. Hartley, Biometrika Tables for Statısticians (University Press, Cambridge, 1957). 
exists if the measurements are independent. If we let

$$
V=\sum_{j=1}^{m} \frac{r_{j}^{2}}{\operatorname{var}\left(y_{j}\right)}
$$

and we let $V_{N 1}$ be the value of $V$ when an equation of $N_{1}$ terms is fitted to $m$ data points, and $\mathrm{V}_{\mathrm{N} 2}$ be the value of $\mathrm{V}$ when an equation of $\mathrm{N}_{2}$ terms is fitted, and $\mathrm{N}_{1}>\mathrm{N}_{2}$

$$
F=\frac{V_{N 2}-V_{N 1}}{V_{N 1}} \quad \frac{n-N_{1}}{N_{1}-N_{2}} .
$$

A value of $F$ significantly greater than one is statistical evidence that the $\mathrm{N}_{1}-\mathrm{N}_{2}$ additional terms were more than fortuitously helpful. Tables giving the significance of values of $F$ in terms of probability of occurrence due to pure chance are given in many collections. 5,6 If the exact form of the additional terms is not known, unfortunately, this approach cannot be used.

Von Holdt ${ }^{7}$ has published a computational method for use with digital calculators having a limıted number of significant figures. Such calculators may lose information during the course of the calculation, since the differences involved in computing determinants, or inverse matrices, are sometimes quite small compared to the numbers involved, if the difference

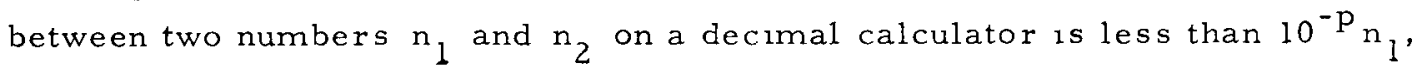
where $p$ is the number of figures the calculator will hold, it will be "rounded" to zero and all information will be lost The number of significant figures retained in the calculation $n_{1}-n_{2}$ is of the order of $p-\log _{10} \frac{N_{1}}{N_{1}-N_{2}}$. Von Holdt has shown that his computational method, although somewhat more laborious than more orthodox techniques, gives only half the figure loss, if one uses an eight-digit calculator, and the data are such that the ordinary method of solution gives a complete loss of information due to rounding errors, von Holdt's method will give results in which the rounding error is only one part in ten thousand. The inverse matrix of the normal equation is also obtained with less rounding error than that encountered when using more usual inversion techniques.

The technique is as follows we write the weighted equations for the data in matrix form as

$$
A X=Y
$$

${ }^{7}$ R. E. von Holdt, Proc. Western Computer Conf. (1955), p. 162. 
where

$$
\begin{aligned}
& A_{1 J}=\frac{a_{1 j}}{\left[\operatorname{var}\left(y_{j}\right)\right]^{1 / 2}}, \\
& X_{1}=x_{1}, \\
& Y_{J}=\frac{y_{j}}{\left[\operatorname{var}\left(y_{j}\right)\right]^{1 / 2}} .
\end{aligned}
$$

We then transform the matrix $A$ as follows. to each column after the first add a suitable multiple $k_{l_{1}}$ of the first column so that the transformed columns are each orthogonal to the first column. This multiple is determined from the orthogonality requirement, 1.e.,

$$
\sum_{j=1}^{m} a_{j]}\left(a_{j l}+a_{j l} k_{11}\right)=0,
$$

consequently

$$
k_{11}=-\frac{\sum_{j=1} a_{j l} a_{j l}}{\sum_{j=1}^{m} a_{j l}^{2}} .
$$

The matrix is transformed again in a similar fashion by adding multiples $k_{2}$ of the modified second column so that each column subsequent to the new second column is orthogonal to it, it is easy to show that orthogonality to the first column is not destroyed by this procedure. This transformation is continued until each column in turn has been used as a bast, and all the columns of the matrix are mutually orthogonal.

It can be seen from the rules of matrix multiplication that the orthogonal ization process is equivalent to multiplying the matrix A from the right by an upper triangular matrix $U$ with unit diagonal elements to give a new matrix $H$ so that

$$
\mathrm{H}=\mathrm{AU} . \quad \therefore \mathrm{H}^{\mathrm{t}}=\mathrm{U}^{\mathrm{t}} \mathrm{A}^{\mathrm{t}} \text {. }
$$

The matrix $U$ can be very simply obtained by treating a unit matrix of $n$ columns by the same transformations as A, using the same multiplying 1actors $k_{j l}$.

Now writing the normal equation in the usual way, and remembering that $\mathrm{UU}^{-1}=\mathrm{I}$, 


$$
\begin{aligned}
& A^{t} A X=A^{t} Y, \\
& U^{t} A^{t} A U U^{-1} X=U^{t} A^{t} Y, \\
& H^{t} H U^{-1} X=U^{t} A^{t} Y .
\end{aligned}
$$

Since the columns of $\mathrm{H}$ are all mutually orthogonal,

$$
\mathrm{H}^{\mathrm{t}} \mathrm{H}=\mathrm{D}
$$

where $\mathrm{D}$ is a diagonal matrix.

$$
\mathrm{X}=\mathrm{UD}^{-1} \mathrm{U}^{\mathrm{t}} \mathrm{A}^{\mathrm{t}} \mathrm{Y}
$$

which is our required solution. The reduction in rounding error arises from the fact that invertıng a diagonal matrix involves nothing more than replacing each diagonal element by its reciprocal, there are no subtractions

Furthermore, since

$$
X-\left(A^{t} A\right)^{-1} A^{t} Y,
$$

we can see from III $184 \mathrm{f}$ and III $184 \mathrm{e}$ that

$$
\left(A^{t} A\right)^{-1}-U D^{-1} U^{t}
$$

Von Holdt ${ }^{7}$ also shows that if the matrix A is augmented, or expanded, by including an extra column equal to the vector $Y$, and this vector also 1 s made orthogonal to each column in turn, it is transformed into a vector $R$ such that

$$
R_{J}=-\frac{r_{j}}{\left[\operatorname{var}\left(y_{j}\right)\right]^{1 / 2}}
$$

or in other words, the components of $R$ are the weighted residuals of the system, obtanned even before the parameters are avalable to compute the points It is then a simple matter to compute

$$
x^{2}=R^{t} R \text {. }
$$

His argument is geometrical the matrix form of the original equation can be taken to be an attempt to express a vector $Y$ by the linear combination of a set of vectors which are the columns of the matrix A. This set of fectors defines a subspace $S$ of $m-$ dimensional Euclidean space spanned by these vectors. Since the computed Y is a linear combination of these vectors it must lie in $\mathrm{S}$, the vector $\mathrm{R}$ computed by orthogonal1zing the vector of the observed $Y$ is orthogonal to the $m$ orthogonal vectors defining $S$ and is therefore the component of $\mathrm{Y}$ (observed) orthogonal to S Consequently,

$$
\text { R - Y (observed) - Y (computed) }
$$

by vector subtraction in a space of $m+1$ dimensions. 


\section{STATISTICS OF COUNTING DATA}

\section{Nature of the Problems}

In order to apply proper statistical calculations to counting data it is necessary to have information on the variances of such data A number of authors have described the simpler statistical problems associated with the counting of radioactive samples, in general following the treatment of Rainwater and $W u{ }^{8}$ These authors discussed in considerable detail the following problems: a) If the expected value for a count is $u$, what is the probability $P_{n}(u)$ that exactly $n$ counts will be observed? b) If exactly $n$ counts are observed, what is the probability that the "true," or expected, value lies between $u$ and $u+d u$ ?

Our treatment of the above problems will in general follow that of Rainwater and $\mathrm{Wu}$. We shall, however, endeavor to extend and refine their treatment somewhat by eliminating some approximations. We shall also consider briefly the statistical problems posed by the existence of counter background, and also the problem of the precision of the result of a measurement of a parameter of a nuclear reaction by counting a radioactive product.

\section{Distribution Functions of Counting Results}

Let us assume that in a sample of radioactive material there are Natoms present at the start of the counting period, and that each of them has the same probability $p$ of disintegrating and producing a count during the counting interval. Probability $p$ is the product of the probabilities: 1) that a given atom will disintegrate during the counting period, and 2) that an atom which disintegrates in the sample will produce a count in the detector. If there is only one species present, it is apparent from the integrated form of the fundamental law of radioactive decay (II.2) that if the number of the atoms in the sample at the start of the measurement is $N$, and the duration of the measurement is $t$, the expected number of atoms still present, 1.e., the number which have not decayed, is $\mathrm{Ne}^{-\lambda t}$, therefore the fraction of atoms expected to decay during the measurement is $\left(1-e^{-\lambda t}\right)$. This is the same as the probability that one atom will decay during the time $t$. The probability that an atom which disintegrates in the sample will produce a count in the detector is the counting efficiency of the counter, which of course depends on

\footnotetext{
L. J. Rainwater and C. S. Wu, Nucleonics, October 1947, p 60.
} 
the decay scheme of the species in question, the geometry of the counting arrangement, and the radiation scattering and absorbing properties of the environment. Either or both of these two factors may be very small so that usually $\mathrm{p}$ is much less than 1 . We now wish to calculate the probability $\mathrm{P}_{\mathrm{n}}$ that precisely $n$ counts will be recorded during the counting interval

The probability that $\mathrm{n}$ consecutive atoms will produce counts is $\mathrm{p}^{\mathrm{n}}$, the probability that the remaining $N$ - n atoms will not produce counts is $(1-p)^{N-n}$, and the probability of recording exactly $n$ events is the sum of the products of these two probabilities for all possible groups of $\mathrm{n}$ atoms chosen from the sample $N$. The number of ways of choosing $n$ atoms from a sample of $\mathrm{N}$ atoms is

$$
C_{N}(n)=\frac{N !}{n !(N-n) !}
$$

and therefore

$$
P_{n}=p^{n}(1-p)^{N-n} \frac{N !}{n !(N-n) !}
$$

This is known as the Bernoulli distribution

Consider the binomial expansion

$$
[p+(1-p)]^{N} \equiv 1
$$

It can easily be seen that the terms of this expansion correspond to the values of $P_{n}$ for all $n$ from 0 to $N$, so that

$$
\sum_{n=0}^{N} P_{n}=1
$$

The expectation value of $n$ is $E(n)$,

$$
E(n)=\sum_{n=0}^{N} n P_{n}=\sum_{n=1}^{N} n P_{n},
$$

$E(n)=\sum_{n=1}^{N} n \frac{p^{n}(1-p)^{N-n} N !}{n !(N-n) !}=p N \sum_{n=1}^{N} \frac{p^{(n-1)}(1-p)^{N-n}}{(n-1) !(N-n) !}(N-1) !$. 
If we let $M=N-1$ and $m=n-1$, then $M-m=N-n$ and

$$
\begin{aligned}
& E(n)=p N \sum_{m=0}^{M} \frac{p^{m}(1-p)^{M-m} M !}{m !(M-m)^{1}}=p N \cdot 1, \\
& E(n)=p N,
\end{aligned}
$$

as one might anticipate. We can substitute in the Bernoullidistribution IV 2 to calculate the probability of observing $n$ events, given $p$ and the expected value $E(n)$.

In order to calculate the variance of $n$ from its distribution function, we calculate

$$
E\left(n^{2}\right)=\sum_{n=0}^{N} n^{2} P_{n}=\sum_{n=1}^{N} n^{2} P_{n}
$$

and making the same substitutions,

$$
E\left(n^{2}\right)=N p \sum_{m=0}^{M}(m+1) \frac{p^{m}(1-p)^{M-m} M !}{m !(M-m) !},
$$

$E\left(n^{2}\right)=N p\left[\sum_{m=0}^{M} m \cdot \frac{p^{m}(1-p)^{M-m} M !}{m !(M-m) !}+\sum_{m=0}^{M} \frac{p^{m}(1-p)^{M-m} M !}{m !(M-m) !}\right]$,

which by comparison with IV.4, IV $5 \mathrm{~b}$, and IV. $5 \mathrm{~d}$ gives

$$
E\left(n^{2}\right)=N p(M p+1)=N p[N p+(1-p)] \text {. }
$$

Recalling that

$$
\begin{aligned}
& \operatorname{var}(n)=E\left(n^{2}\right)-E(n)^{2}, \\
& \operatorname{var}(n)=N p(1-p)=(1-p) E(n),
\end{aligned}
$$

so that the standard deviation $\sigma_{n}$ of $n$ is given by

$$
\sigma_{n}=\sqrt{(1-p) E(n)}
$$

Since $p$ is usually very small, it can usually be neglected. 
The Bernoulli distribution function given by equation IV.2 can be considerably simplified in form in those cases where $N$ is very large, $p$ is very small, and $n$ is very much less than $N$; fortunately this is often true. We make the approximations

$$
\begin{aligned}
& \frac{N !}{(N-n) !}=N(N-1)(N-2) \ldots(N-n+1) \approx N^{n} \\
& (1-p)^{N-n} \approx e^{-p(N-n)} \approx e^{-p E(n)} .
\end{aligned}
$$

Inserting these and equation IV. $5 \mathrm{~d}$ into equation IV.2 we get what is known as the "Poisson distribution,"

$$
P_{n}=\frac{\overline{E(n)} n e^{-E(n)}}{n !}
$$

As might be expected from the approximations involved in its derivation, this distribution gives

$$
\begin{aligned}
\operatorname{var}(n) & =E(n), \\
\therefore \sigma_{n} & =\sqrt{E(n)}
\end{aligned}
$$

For cases where the Poisson distribution is a valid approximation to the Bernoulli distribution, and $n$ is quite large, the Poisson distribution may be well approximated by the Gaussian, or normal, distribution. This approximation is obtained by replacing n! by Stirling's approximation, and by transforming the variable $n$ to $x=[n-E(n)]$. We then have

$$
\begin{aligned}
& P_{x}=\frac{[E(n)]^{E(n)+x} \cdot e^{-E(n)}}{[E(n)+x] !}=\frac{[E(n)]^{E(n)} \cdot[E(n)]^{x} e^{-E(n)}}{[E(n)] ![E(n)+1][E(n)+2] \ldots[E(n)+x]}, \\
& P_{x}=\frac{[E(n)]^{E(n)} e^{-E(n)}}{[E(n)] !} \cdot \frac{[E(n)]^{x}}{[E(n)+1][E(n)+2] \ldots[E(n)+x]} \\
& {[E(n)] ! \approx \sqrt{2 \pi E(n)} \cdot\left[\frac{E(n)}{e}\right]^{E(n)},}
\end{aligned}
$$


$\therefore P_{x}=\frac{1}{\sqrt{2 \pi E(n)}} \cdot \frac{1}{\left[1+\frac{1}{E(n)}\right]\left[1+\frac{2}{E(n)}\right] \cdots\left[1+\frac{x}{E(n)}\right]}$.

Since $n$ is large, $n-E(n)$ will be small compared to $n$, so that we can approximate

$$
\left[1+\frac{z}{E(n)}\right] \approx e^{z / E(n)} \text { when }|z| \leq|x|
$$

Consequently,

$[1+1 / E(n)][1+2 / E(n)] \ldots\left[1+\frac{x}{E(n)}\right] \approx \exp \left[\sum_{m=1}^{x} m / E(n)\right]=e^{\left(x^{2}+x\right) / 2 E(n)}$.

Neglecting $x$ compared to $x^{2}$, we have finally

$$
P_{x}=\frac{1}{\sqrt{2 \pi E(n)}} e^{-x^{2} / 2 E(n)}
$$

or

$$
P_{x}=\frac{1}{\sqrt{2 \pi \sigma_{x}^{2}}} e^{-x^{2} / 2 \sigma_{x}^{2}}
$$

which is the normal distribution with $\operatorname{var}(x)=E(n)$.

\section{Distribution of Expected Values for a Given Observed Count - Small Sampling}

We can attack the inverse problem very easily. We now wish to know the expected value of $n$, and its variance, if we have an actual observed value $n$.

This is most simply approached for the case in which the Poisson distribution is applicable. We rewrite equation IV 12, letting the symbol $u$ stand for $E(n)$ for convenience,

$$
P_{n}(u)=\frac{u^{n} e^{-u}}{n !}
$$

to show that $P_{n}(u)$ is a function both of $u$ and of $n$. 
We now state the problem: for an actual count $n$, what is the probability that the actual value $x$ of $u$ lies between $u$ and $u+d u$ ? The question may be rephrased: if we consider repeating the identical experiment a large number of times, what is the predicted value of the average result given that the result of a single experiment is $\mathrm{n}$; and how reliable is the prediction, that is, what is its variance? In cases where the number of counts is large, it can be seen that equation IV. 15 is the solution of the inverse problem when written

$$
P_{n}(x) d x=\frac{x^{n} e^{-x}}{n !} d x
$$

From this we get the expected value of $\mathrm{x}$ from

$$
\begin{aligned}
E(x) & =\int_{0}^{\infty} x P_{n}(x) d x=\frac{1}{n !} \int_{0}^{\infty} x^{n+1} e^{-x} d x=\frac{(n+1) !}{n !}, \\
\therefore & E(x)=n+1 .
\end{aligned}
$$

The fact that the expected value is larger than the observed value is surprising at first, until we consider that even if the observed value is zero, the expected value need not be zero.

Calculation of the variance of $\mathrm{x}$ gives

$$
\begin{aligned}
& \operatorname{var}(x)=\int_{0}^{\infty} x^{2} P_{n}(x) d x-\overline{E(x)}^{2}, \\
& \operatorname{var}(x)=\frac{(n+2) !}{n !}-(n+1)^{2}=(n+2)(n+1)-(n+1)^{2}, \\
& \operatorname{var}(x)=n+1=E(x) .
\end{aligned}
$$

\section{Distribution of Expected Values for a Given Observed Count - Large}

\section{Sampling}

Rainwater and $\mathrm{Wu}^{8}$ did not consider the problem of predicting expected count and count variance for cases where an appreciable fraction of the atoms present might be expected to give rise to counts. For such a case to arise, two conditions must be met simultaneously. First, the counting equipment must have a high efficiency for detection, which includes both a high solid angle for radiation from the sample to enter the counter and a high probability 
that entering radiation will cause a pulse in the counting circuit. Second, the counting period must be at least an appreciable fraction of a half-life of the species involved. Such conditions are met in experiments involving shortlived species formed in low yield, where in order to obtain data of maximum reliability it is necessary to usehigh-efficiency counters and count for extended periods. Experiments designed to detect minimal amounts of certain contaminants may also be conducted under these conditions, where very long counts are resorted to.

Under these conditions the as sumptions which lead to the Poisson distribution are no longer valid. We must therefore work with the more accurate but less tractable Bernoulli distribution. Our problem remains the same as in the previous section: Given an observed number of events $n$, what is the expected average number of events during a number of identical experiments, and what is the reliability of that number?

We approach the problem via a conceptual experiment We assume that the experiment is carried out a very large number of times with a variety of different numbers of atoms of material. Let the number of times the experiment is performed be $w$ and the probability that the number $N$ of atoms in a given experiment has the value $z$ be $\pi_{N}(z)$. Let $w$ be so large that the number of experiments conducted in which $N$ has the exact value $z$ be $w \pi_{N}(z)$ as precisely as we choose.

Then if $\mathrm{N}$ has the value $\mathrm{z}$, the probability that the number of counts observed, $x$, has the precise value $n$ is given by the Bernoulis distribution.

$$
P_{x}(n, z)=\frac{p^{n}(1-p)^{z-n} z !}{n !(z-n) !}
$$

where $p$ is, as before, the probability that a single atom will decay and give rise to a count during the course of the experiment.

The number of times this result will be observed in w experiments, if w is sufficiently large, is

$$
m_{x}(n, z)=w \pi_{N}(z) P_{x}(n, z)
$$

We know that if $\mathrm{n}$ events were observed there were at least $\mathrm{n}$ atoms in the sample. Therefore the total number of experiments, for any value of 
$N$ equal to or greater than $n$, which will give rise to a value $n$ for $x$ is

$$
M_{x}(n)=\sum_{z=n}^{\infty} w \pi_{N}(z) P_{x}(n, z)
$$

Consequently, the fraction of the experiments leading to the value $\mathrm{n}$ for $\mathrm{x}$ in which $\mathrm{N}$ had the value $\mathrm{z} 1 \mathrm{~s}$ given by the ratio of equations IV 19 and IV.20. If $w$ is sufficiently large, this constitutes a definition of probability, so that

$$
P_{N}(z)=\frac{w \pi_{N}(z) P_{x}(n, z)}{\sum_{z=n}^{\infty} w \pi_{N}(z) P_{x}(n, z)}
$$

or

$$
P_{N}(z)=\frac{p^{n}(1-p)^{z-n} z ! \pi_{N}(z)}{n !(z-n) ! \sum_{z=n}^{\infty} \frac{p^{n}(1-p)^{z-n} z !}{n !(z-n) !} \pi_{N}(z)}
$$

or, simplifying,

$$
P_{N}(z)=\frac{z !(1-p)^{z}}{(z-n) !} \cdot \frac{\pi_{N}(z)}{\sum_{z=n}^{\infty} \frac{z !(1-p)^{z}}{(z-n)^{1}} \cdot \pi_{N}(z)} .
$$

In order to proceed further it is necessary to make an assumption. We assume, lacking any other information, that any value of $N$ on our series of w experiments is as likely as any other, or alternatively, we assume that if we conduct our experiments in such a way that all values of $\mathrm{N}$ a re equally likely, the conclusions drawn from such a set of experiments may be applied to actual laboratory results.

If we make such an assumption, the $\pi_{N}(z)$ are all equal and will cancel. In that case we are left with an expression

$$
P_{N}(z)=\frac{z !(z-p)^{z}}{(z-n) ! \sum_{z=n}^{\infty} \frac{z !(1-p)^{z}}{(z-n) !}}
$$

which is obviously normalized. 
It is necessary to evaluate the sum of the infinite series in the denominator. The value of this series depends only on the constants $n$ and $p$. Let us define the symbol

$$
Q_{n}=\sum_{z=n}^{\infty} \frac{z !(1-p)^{z}}{(z-n) !}
$$

If $\mathrm{n}$ is zero, then

$$
Q_{0}=\sum_{z=0}^{\infty}(1-p)^{z}=1+(1-p)+(1-p)^{2}+(1-p)^{3}+\ldots
$$

Since $1-p$ is less than one, this is a convergent geometric series with a ratio 1 - p, and

$$
Q_{0}=\frac{1}{1-(1-p)}=\frac{1}{p} \text {. }
$$

Let us now consider $Q_{m+]}$, and let $y=z-l$ or $z=y+1$.

$$
Q_{m+1}=\sum_{z=m+1}^{\infty} \frac{z !(1-p)^{z}}{[z-(m+1)] !}=\sum_{y=m}^{\infty} \frac{(y+1) !(1-p)^{y+1}}{(y+1-m-1) !}
$$

$Q_{m+1}=(1-p) \sum_{y=m}^{\infty}(y+1) \cdot \frac{y !(1-p)^{y}}{(y-m) !}$

$Q_{m+1}=(1-p)\left[\sum_{y=m}^{\infty} y \cdot \frac{y !(1-p)^{y}}{(y-m) !}+\sum_{y=m}^{\infty} \frac{y !(1-p)^{y}}{(y-m) !}\right]$

$Q_{m+1}=(1-p)\left[\sum_{y=m}^{\infty}(y-m+m) \frac{y !(1-p)^{y}}{(y-m) !}+Q_{m}\right]$,

$Q_{m+1}=(1-p)\left[\sum_{y=m}^{\infty}(y-m) \cdot \frac{y !(1-p)^{y}}{(y-m) !}+m \sum_{y=m}^{\infty} \frac{y !(1-p)^{y}}{(y-m) !}+Q_{m}\right]$, 
$Q_{m+1}=(1-p)\left[\sum_{y=m+1}^{\infty}(y-m) \frac{y !(1-p)^{y}}{(y-m) !}+(m+1) Q_{m}\right]$.

Note the change in the lower limit on the summation sign. This is allowable, since the first term when $y=m$ is zero. Dividing $(y-m)$, which is not zero, into the numerator and denominator in the summation,

$$
Q_{m+1}=(1-p)\left\{\sum_{y=m+1}^{\infty} \frac{y !(1-p)^{y}}{[y-(m+1)]^{1}}+(m+1) Q_{m}\right\}
$$

or

$$
Q_{m+1}=(1-p)\left[Q_{m+1}+(m+1) Q_{m}\right]
$$

which gives, immediately,

$$
Q_{m+1}=\left(\frac{1-p}{p}\right)(m+1) Q_{m}
$$

and since $Q_{0}=1 / p$ from equation IV.27,

$$
Q_{y}=\left(\frac{1-p}{p}\right)^{y} \cdot \frac{y !}{p} \text {. }
$$

It should be noted that equation IV.28c can be transformed into a useful form

$$
\sum_{y=m}^{\infty} y \cdot \frac{y !(1-p)^{y}}{(y-m) !}=\frac{Q_{m+1}}{1-p}-Q_{m}=\frac{m+1-p}{p} Q_{m}
$$

We now wish to get the expected value and variance of the number of atoms in the sample, as well as the expected value and variance of the number of counts in the counter. The expected value of $\mathrm{N}$ is

$$
E(N)=\sum_{z=n}^{\infty} z P_{N}(z)=\sum_{z=n}^{\infty} z \cdot \frac{z !(1-p)^{z}}{(z-n) ! Q_{n}}
$$

substituting $Q_{n}$ for the equivalent summation in equation IV 24 . We then have, substituting in equation IV 30 from equation IV.29, 


$$
\begin{aligned}
& E(N)=\frac{1}{Q_{n}} \sum_{z=u}^{\infty} z \cdot \frac{z !(1-p)^{z}}{(z-n) !}=\frac{1}{Q_{n}}\left(\frac{n+1-p}{p} \cdot Q_{n}\right), \\
& E(N)=\frac{n+1-p}{p} .
\end{aligned}
$$

The variance of $\mathrm{N}$ is obtained from the relationship

$$
\operatorname{var}(N)=E\left(N^{2}\right)-\overline{E(N)}^{2}
$$

$E\left(N^{2}\right)=\sum_{z=n}^{\infty} z^{2} P_{N}(z)=\frac{1}{Q_{n}} \sum_{z=n}^{\infty} z^{2} \frac{z !(1-p)^{z}}{(z-n) !}$

$E\left(N^{2}\right)=\frac{1}{Q_{n}} \sum_{z=n}^{\infty}\left[z^{2}-(2 n+1) z+n(n+1)+(2 n+1) z-n(n+1)\right] \frac{z !(1-p)^{z}}{(z-n) !}$,

$$
\begin{aligned}
E\left(N^{2}\right)=\frac{1}{Q_{n}} \sum_{z=n}^{\infty}(z-n)[z-(n+1)] \frac{z !(1-p)^{z}}{(z-n) !} & \\
& +\frac{(2 n+1)}{Q_{n}} \sum_{z=n}^{\infty} z \frac{z !(1-p)^{z}}{(z-n) !}-n(n+1)
\end{aligned}
$$

$$
\begin{array}{r}
E\left(N^{2}\right)=\frac{1}{Q_{n}} \sum_{z=n+2}^{\infty}(z-n)[z-(n+1)] \cdot \frac{z !(1-p)^{z}}{(z-n) !}+(2 n+1)\left(\frac{n+1-p}{p}\right) \\
-n(n+1), \quad(I V)
\end{array}
$$

$$
\begin{aligned}
E\left(N^{2}\right)=\frac{1}{Q_{n}} \sum_{z=n+2}^{\infty} \frac{z !(1-p)^{z}}{[z-(n+2)] !} & +\left[\frac{(2 n+1)(n+1)}{p}-\left(n^{2}+3 n+1\right)\right], \\
E\left(N^{2}\right)=\frac{Q_{n+2}}{Q_{n}}+\left[\frac{(2 n+1)(n+1)}{p}-\left(n^{2}+3 n+1\right)\right]=\left(\frac{1-p}{p}\right)^{2}(n+2)(n+1) & {\left[-\left[\frac{(2 n+1)(n+1)}{p}-\left(n^{2}+3 n+1\right)\right],\right.}
\end{aligned}
$$


$E\left(N^{2}\right)=\frac{(n+2)(n+1)}{p^{2}}-\frac{2(n+2)(n+1)-(2 n+1)(n+1)}{p}+(n+2)(n+1)$ $-n^{2}-3 n-1,(\operatorname{IV} 33 h)$

$E\left(N^{2}\right)=\frac{n+1}{p^{2}}+\frac{(n+1)^{2}}{p^{2}}-\frac{3(n+1)}{p}+1$

Therefore

$$
\begin{aligned}
& \operatorname{var}(N)=\frac{n+1}{p^{2}}+\left(\frac{n+1}{p}\right)^{2}-\frac{3(n+1)}{p}+1-\left(\frac{n+1}{p}-1\right)^{2}, \\
& \operatorname{var}(N)=\frac{n+1}{p^{2}}-\frac{n+1}{p}, \\
& \operatorname{var}(N)=\frac{(n+1)(1-p)}{p^{2}} .
\end{aligned}
$$

In order to determine $\mathrm{E}(\mathrm{n})$, we recall that for the Bernoulli distribution

$$
\begin{aligned}
& E(n)=E(p N)=p E(N) . \\
& E(n)=u=n+1-p
\end{aligned}
$$

which may be compared with equation IV.J7b, derived from the Poisson distribution. It will be seen that the approximations involved in converting the Bernoulli distribution to the Poisson distribution result in the loss of the term (-p), it is easy to see that if $\mathrm{p}$ approaches 1 (that is, if any atoms present are nearly certain to be counted) then the expected value of the count approaches the value of the count itself, for example, it is no longer likely that the expected value will be much greater than zero when an actual observation of zero is recorded.

The variance of $\mathrm{n}$ is calculated from

$$
\begin{aligned}
& \operatorname{var}(n)=\operatorname{var}(p N)=p^{2} \operatorname{var}(N) \\
& \operatorname{var}(n)=(1-p)(n+1)
\end{aligned}
$$

which differs from the variance deduced from the Poisson distribution by the factor $(1-p)$. It is obvious that when $p$ is one (that 15 , that every atom present is certain to count), that the result is not subject to variation, hence its variance must be zero, as it is, the number of atoms in the sample can be measured with certaintv. 


\section{Correction for Counter Background}

It can be shown (see Rainwater and $\mathrm{Wu}^{8}$ ) that in cases where the Poisson distribution can be used it is not necessary to assume that $p$ is constant for every atom, in other words the presence of many different species in the sample does not affect our statistical conclusions. If we consider the counter background to arise from a "species" with a very long half-life, its expected value and variance will be given by IV.17b and IV.17e. A word of caution is in order. if the counting equipment is malfunctioning in any of a number of ways to give rise to a nonrandom background, the results no longer apply, in particular as regards variance. If the background is random, then a measurement of background is independent of the gross count taken on the sample, in the statistical sense, consequently the covariance of the two measurements is zero, and the variance of a net count after subtracting the result of a background measurement is the sum of the variances of the background measurement and the gross count.

When the Poisson distribution is not applicable to the sample, the situation becomes more complicated. Fortunately, the Poisson distribution is always applicable to the background. It is necessary to take a background measurement, to deduce from this result the expected background count (which is the observed count plus one) and to calculate the probability of each possible value $b$ of the background $P_{B}(b)$ from zero to $g$, where $g$ is the gross count recorded for the sample. Each such probability is then normalized by the sum of all the probabilities $\sum_{b=0}^{g} P_{B}(b)$, they must be normalized from the Poisson distribution since the background count cannot take on all possible values, negative values of the net count are not possible If the number of events in the gross count, $g$, is considerably larger than the background count the normalizing factor will be very nearly one, but for counts not greatly in excess of background it is very different from one.

Each renormalized probability that the background count had the value $b$ is now the probability that in the gross count the count $X$ due to the sample had the value $g$ - $b$, which may be written

$$
\frac{1}{R_{g}} \cdot P_{B}(b)=P_{x}(g-b)
$$


where $l / R_{g}$ is the renormalizing factor,

$$
\frac{1}{R_{g}}=\frac{1}{\sum_{b=0}^{g} P_{B}(b)} \text {, }
$$

and $P_{B}(b)$ is calculated from the Poisson distribution IV.12, with $u=E(n)$ taken as the observed number of events during the measurement, plus one, and $\mathrm{n}$ taken to be each value of $\mathrm{b}$ in turn.

We then calculate $E(N)$ and $\operatorname{var}(N)$, or $E(n)$ and $\operatorname{var}(n)$, as desired, from equations IV. 32 and IV.34c or IV. 36 and IV.38, for sample count by multiplying each expected value by $P_{x}(g-b)$ and summing over all values. Let the observed background count (over the same period) be $\beta$; then

$$
u=\beta+1=E(B)
$$

and

$$
R_{g}(u)=\sum_{j=0}^{g} \frac{u^{j} e^{-u}}{j !} \longrightarrow 1 \text { as } g / u \longrightarrow \infty
$$

Then

$$
\begin{aligned}
& E(N)=\sum_{b=0}^{g}\left(\frac{n+1-p}{p}\right) \frac{u^{g-n} e^{-u}}{(g-n) !} \cdot \frac{1}{R_{g}(u)}, \\
& E(N)=\sum_{b=0}^{g}\left(\frac{g+1-p}{p}-\frac{b}{p}\right) \frac{u^{b} e^{-u}}{b !} \cdot \frac{1}{R_{g}(u)}, \\
& E(N)=\frac{g+1-p}{p}-\frac{1}{p} \sum_{b=0}^{g} \frac{u^{b} e^{-u}}{b !} \cdot \frac{1}{R_{g}(u)}, \\
& E(N)=\frac{g+1-p}{p}-\frac{1}{p} \sum_{b=1}^{g} \frac{u^{b} e^{-u}}{(b-1) !} \cdot \frac{1}{R_{g}(u)} .
\end{aligned}
$$


Let $y=j-b$, then

$$
E(N)=\frac{g+1-p}{p}-\frac{u}{p} \cdot \frac{R_{g-1}(u)}{R_{g}(u)}=\frac{g+1-p-u}{p}+\frac{u}{p} \cdot \frac{u^{g} e^{-u}}{g ! R_{g}(u)} \cdot
$$

(IV.43e)

When the gross count is sufficiently above the background, the last term becomes very small and may be neglected In this case

$$
E(N) \approx \frac{g+1-p-u}{p}=\frac{g-\beta-p}{p} .
$$

If the observed background count is taken over a period $t_{b}$ of different length than the duration of the gross count ${ } g$,

$$
E(N) \approx \frac{g+1-p-\frac{t g}{t_{b}}(\beta+1)}{p}
$$

since we expect the background count to be uniformly distributed in time.

The variance of $\mathrm{N}$ is calculated from equation IV. 33 .

$$
\begin{aligned}
& E\left(N^{2}\right)=\sum_{b=0}^{g}\left[\frac{n+1}{p^{2}}+\frac{(n+1)^{2}}{p^{2}}-\frac{3(n+1)}{p}+1\right] \frac{u^{b} e^{-u}}{b ! R_{g}(u)}, \\
& E\left(N^{2}\right)=\sum_{b=0}^{g}\left[\frac{g-b+1}{p^{2}}+\frac{(g-b+1)^{2}}{p^{2}}-\frac{3(g-b+1)}{p}+1\right] \frac{u^{b} e^{-u}}{b ! R_{g}(u)},
\end{aligned}
$$

$\operatorname{var}(\mathrm{N})=E\left(\mathrm{~N}^{2}\right)-\overline{\mathrm{E}(\mathrm{N})}^{2}$.

Going through the algebra as before, and remembering that

$$
\sum_{b=0}^{g} b \frac{u^{b} e^{-u}}{b !}=\sum_{b=1}^{g} b \frac{u^{b} e^{-u}}{b !}=\sum_{b=1}^{g} \frac{u^{b} e^{-u}}{(b-1) !}=u \sum_{(b-1)=0}^{g-1} \frac{u^{(b-1)} e^{-u}}{(b-1) !}=u R_{g-1}(u)
$$

$$
\begin{array}{r}
\sum_{b=0}^{g} b^{2} \frac{u^{b} e^{-u}}{b !}=\sum_{b=1}^{g} b \cdot \frac{u^{b} e^{-u}}{(b-1) !}=\sum_{(b-1)=0}^{g-1}[(b-1)+1] \frac{u^{b-1+1} e^{-u}}{(b-1) !} \\
=u R_{g-1}(u)+u^{2} R_{g-2}(u)
\end{array}
$$


we finally get, after a certain amount of algebra,

$\operatorname{var}(N)=\frac{(1-p)(g+1)}{p^{2}}+\frac{u}{p} \frac{R g-1(u)}{R_{g}(u)}+\frac{u^{2}}{p^{2}}\left[\frac{R_{g-2}(u)}{R_{g}(u)}-\frac{R_{g-1}(u)}{R_{g}(u)}\right]^{2}$.

If, as before, $g \gg u$,

$$
\operatorname{var}(N) \approx \frac{(1-p)(g+1)+u p}{p^{2}} .
$$

As before, when $g \gg \mathrm{u}$,

$E(n)=E(p N)=p E(N) \approx g-\beta-p$,

$\operatorname{var}(n)=\operatorname{var}(p N)=p^{2} \operatorname{var}(N) \approx(1-p)(g+1)+u p=(1-p)(g+1)+p(\beta+1)$

When $g$ is not much greater than $u$, the $R_{g}(u), R_{g-1}(u)$, and $R_{g-2}(u)$ must be evaluated and the full equations derived from equations IV.4 $3 \mathrm{e}$ and IV.48 must be used. This procedure is extremely laborious. Fortunately, we are frequently concerned with the parameters of the reaction forming the active species, rather than with the actual numbers of atoms in the sample as will be shown, the Poisson distribution is always applicable in such a case.

\section{Errors of Extrapolation}

It rarely happens that counting can be started immediately after the creation of the radioactive species. If some delay occurs between the time of interest to the experimenter, $t_{0}$, and the time of the measurement, $t_{\lambda}$, one can extrapolate back to $t_{0}$ by multiplying by the exponential factor $e^{\lambda\left(t-t_{0}\right)}$. It is important to realize, however, that no matter how precisely the counting rate at $t$ is known, and no matter how precisely the half-life or decay constant is known, a statistical uncertainty is introduced by the extrapolation itself This is readily seen by considering that the atoms still present at $t$ are those which have not decayed in the interval $t_{0}$ to $t$. Since those which have decayed are subject to statistical fluctuations, the sum of those still present and those which have decayed is subject to such fluctuation.

The variance of the extrapolated number can be derived as follows: Let the number of atoms present at $t_{0}$ be $\mathrm{N}_{0}$, the number of atoms decaying in 
the interval $t_{0}$ to $t$ be $n$, the number of atoms remaining at time $t$ be $N$; the extrapolation factor is then $\mathrm{N}_{0} / \mathrm{N}$.

Now the probability $p$ of one atom decaying in the interval $t_{0}$ to $t$ is $1-e^{-\lambda\left(t-t_{0}\right)}$ where $\lambda$ is the decay constant of the species.

The probability that $n$ atoms decay during the interval $t_{0}$ to $t$ is obtained from the Bernoulli distribution, since $p$ is not necessarily much smaller than ]. We have, therefore,

$$
P_{n}=p^{n}(1-p)^{N_{0}-n} \cdot \frac{N_{0} !}{n_{0} !\left(N_{0}-n\right) !}
$$

as the probability that $\mathrm{n}$ atoms will decay during the interval of interest.

Substituting

$$
\begin{aligned}
& \text { a) } n=N_{0}-N, \\
& \text { b) } p=1-e^{-\lambda\left(t-t_{0}\right)}
\end{aligned}
$$

we get

$$
P_{n}=\left[1-e^{-\lambda\left(t-t_{0}\right)}\right]^{N_{0}-N} \cdot\left(e^{-\lambda t}\right)^{N} \cdot \frac{N_{0} !}{\left(N_{0}-N\right) ! \cdot N !}
$$

Now the probability that $\mathrm{n}$ atoms decay is the same as the probability that $\mathrm{N}$ atoms remain, so that

$$
\mathrm{P}_{\mathrm{N}}=\mathrm{P}_{\mathrm{n}}
$$

The form of the distribution function for $N$ is then seen to be a Bernoulli distribution with a probability $\mathrm{p}^{\prime}=\mathrm{e}^{-\lambda\left(t-t_{0}\right)}$, and from equations IV.32 and IV. $34 \mathrm{c}$,

$$
\begin{aligned}
E\left(N_{0}\right) & =\frac{N+1-p^{\prime}}{p^{\prime}}=(N+1) e^{\lambda\left(t-t_{0}\right)}-1, \\
\operatorname{var}\left(N_{0}\right) & =\frac{(N+1)\left(1-p^{\prime}\right)}{\left(p^{\prime}\right)^{2}}=(N+1)\left[1-e^{-\lambda\left(t-t_{0}\right)}\right] \cdot e^{2 \lambda\left(t-t_{0}\right)} .
\end{aligned}
$$


The extrapolation factor is given by

$F_{e}=E\left(N_{0}\right) / N=\left[\frac{N+1-e^{-\lambda\left(t-t_{0}\right)}}{N}\right] e^{\lambda\left(t-t_{0}\right)}=\frac{1-e^{\lambda\left(t-t_{0}\right)}}{\lambda\left(t-t_{0}\right)}+e^{\lambda\left(t-t_{0}\right)}$

which for $N$ large compared to 1 is simply $e^{\lambda\left(t-t_{0}\right)}$, which is as expected, only for extremely small values of $N$ is there an appreciable correction. The variance of the extrapolation factor is given by

$\operatorname{var}\left(F_{e}\right)-\frac{1}{N^{2}} \operatorname{var}\left(N_{0}\right)=\frac{(N+1)\left[1-e^{-\lambda\left(t-t_{0}\right)}\right] e^{2 \lambda\left(t-t_{0}\right)}}{N^{2}}$

which for values of $N$ large compared to l simplifies to

$$
\operatorname{var}\left(F_{e}\right) \approx\left[\frac{1-e^{-\lambda\left(t-t_{0}\right)}}{N}\right] e^{2 \lambda\left(t-t_{0}\right)}
$$

When extrapolating from a known number of atoms at time $t$, with known variance, back to a desired $t_{i m e} t_{0}$, we have from Theorem $V$, Chapter III,

$$
\begin{aligned}
N_{0} & =N F_{e} \\
\operatorname{var}\left(N_{0}\right) & =\left(N F_{e}\right)^{2}\left[\frac{\operatorname{var}(N)}{N^{2}}+\frac{\operatorname{var}\left(F_{e}\right)}{F_{e}^{2}}\right],
\end{aligned}
$$

and hence, unless $N$ is very small,

$$
\operatorname{var}\left(N_{0}\right) \approx N_{0}^{2}\left[\frac{\operatorname{var}(N)}{N^{2}}+\frac{1-e^{-\lambda\left(t-t_{0}\right)}}{N}\right]
$$

If instead of extrapolating numbers of atoms we adopt the more usual course of extrapolating counts per unit time $A$ at time $t$ back to counts per unit time $\mathrm{A}_{0}$ at time $t_{0}$, if the efficiency of our counter is $\eta$ we have

$$
\begin{aligned}
\mathrm{A} & =\eta \lambda \mathrm{N}, \\
\mathrm{A}_{0} & =\eta \lambda \mathrm{N}_{0}, \\
\mathrm{~A}_{0} & =A F_{\mathrm{e}},
\end{aligned}
$$

and consequently, for $\mathrm{N}$ considerably greater than $\mathrm{l}$,

$$
\operatorname{var}\left(A_{0}\right)=A_{0}^{2}\left[\frac{\operatorname{var}(A)}{A^{2}}+\frac{1-e^{-\lambda\left(t-t_{0}\right)}}{A} \cdot \lambda_{\eta}\right]
$$


The last term within the parenthesis implies that if we wish to know the variance of an extrapolated number, we must know the efficiency of our counter: we are faced with the necessity of absolute counting.

Fortunately, the nature of the expression permits a simplifyıng approximation which in most cases eliminates this requirement. The "extrapolation variance" has an upper limit; since $\eta$ is at most 1 , its asymptotic maximum value at long times is $\lambda / A$. If this value is small compared to $v$ ir $(i s) / A^{2}$ the extrapolation error may be neglected without seriously affecting our estimate of the precision of our answer.

\section{Statistical Reliability of Measurements on Nuclear Reactions}

Let us consider an experiment in which a sample of $M$ atoms is immersed in a flux $\phi$ of particles per unit area per second and left there for a time $t$. The sample is then counted immediately and $n$ events are recorded. It is desired to calculate the cross section $\sigma$ of the reaction, and its variance, from the data.

This problem can most readily be approached by redefining $p$ to be the probability that an atom of original target material will react and subsequently give rise to a count in the apparatus. From this definition it will readily be seen that, in order for $p$ to be appreciably greater than zero, all of the following conditions must be met: (1) An appreciable fraction of the target material must be consumed in the nuclear reaction; (2) the reaction must be carried out in a time short compared to the product half-life; (3) the counting measurement must be carried out over a time long compared to the half-life; (4) a major portion of the sample must be placed in the counter; and (5) the counter must be of high geometry and high efficiency. These conditions are mutually exclusive under present technology, since conditions (1) and (2) require enormous fluxes available only in nuclear explosions and condition (4) is not possible to fulfill following a nuclear explosion. Consequently $p$ is always small, and Poisson statistics always applies when deducing the variance of a nuclear reaction parameter such as flux, reaction cross section, or amount of target material. 


\section{THE RESOLUTION OF DECAY DATA}

\section{Nature of the Problem}

We have seen in Chapter II that the expected value of the activity of a sample of radioactive material changes with time in a manner which may be described by a linear combination of negative exponential functions, that is, functions of the form $\exp \left(-\lambda_{1} t\right)$, where each $\lambda_{1}$ is associated with one of the species present so that for $n$ species present we have $n$ terms, plus a possible constant term if some species are being formed via a nuclear reaction. We have seen further that certain physical properties, such as reactor flux or reaction cross section, may be derived from the values of the coefficients of these exponential terms. It remains to apply the statistical techniques of Chapters III and IV to the resolution of decay data, that is, to determining the best values for such coefficients from a set of experimental observations.

We have seen in Chapter II how these coefficients may be estimated graphically, however, for the best solution to our problem it is necessary to resort to a least-squares solution following methods described in Chapter III, Section 5. The data (obtained as events per unit time) are to be fitted to a function of the form

$$
y_{j}=\sum_{1=1}^{n} x_{1} e^{-\lambda_{1} t} j
$$

where $t_{J}$ is measured at the midpoint of each measured count, or for extreme precision (see Chapter II, Section 8)

$$
y_{j}=\sum_{1=1}^{n} x_{1} e^{-\lambda_{1} t}\left[\frac{\left.1-e^{-\lambda_{1}(\Delta t)}\right]}{\lambda_{1}(\Delta t)}\right]
$$

where $(\Delta t)$, is the duration of the $\underline{j}^{\text {th }}$ counting measurement and $t_{j}$ is measured at the start of the count. The coefficients to be determined are the $\mathrm{x}_{1}$, proper weighting will be discussed below in detail.

We shall assume in this chapter that the $\lambda$ are precisely known for all the species in the system. We shall consider the proper weighting of the data for a least-squares solution, the kind and magnitude of the variances which contribute to the weighting factor, and the effect on the results of any unanticipated variance. The $\lambda$ for our solution are as sumed to be constants, we 
shall investigate the systematic effect on the resolution of slight variations in the values of the $\lambda$. We shall also consider briefly the problem of rejection of data which appear to fall outside the expected range of variation.

In addition, we shall also consider briefly the analysis of replicate samples, the analysis of the sources of variation between replicates, and the proper averaging of replicate results.

\section{The Least-Squares Solution; Weighting of the Data}

We have seen in Chapter III that the squares of the deviations of points $y_{j}$ should be weighted by the reciprocals of the variances of the $y_{j}$ before summing for the least-squares solution; alternatively, each equation describing a bit of data $y_{j}$ should be weighted by the reciprocal of the square root of the variance of $y_{j}$ before commencing a matrix solution.

If every count is recorded, and the counting equipment is perfectly stable, the variance of an observed count is given by equation IV.50b of Chapter IV. In the usual case, the probability of a single atom being counted is negligibly small and the gross number of events recorded is very large; the variance of the event is then the gross number of events, plus the number of background events observed in a measurement of equal duration. Frequently, the background is observed in a measurement of one duration ${ }_{B}$ and the gross count is observed in another of different duration $t_{B}$; if the number of gross and background events are $G$ and $B$ respectively, and the desired net counting rate is $z$, then

$$
z=\frac{G}{{ }^{t} G}-\frac{B}{t_{B}} \equiv g-b,
$$

and

$$
\operatorname{var}(z)=\operatorname{var}(g)+\operatorname{var}(b)
$$

by Theorem IV of Chapter III, since measurements of $g$ and $b$ are independent. By equation III. 14,

$$
\operatorname{var}(z)=\operatorname{var}\left(\frac{G}{t_{G}}\right)+\operatorname{var}\left(\frac{B}{t_{B}}\right)=\frac{1}{t_{G}{ }^{2}} \operatorname{var}(G)-\frac{1}{t_{B}{ }^{2}} \operatorname{var}(B) .
$$

When the Poisson distribution function is applicable,

$$
\operatorname{var}(z)=\frac{G+1}{t_{G}^{2}}+\frac{B+1}{t_{B}^{2}} \text {. }
$$


and when both $G$ and $B$ are large compared to unity,

$$
\operatorname{var}(z)=\frac{g}{t_{G}}+\frac{b}{t_{B}}
$$

If a scaling circuit is used in which the register records only every $\mathrm{S}_{\mathrm{G}}$ events during the gross measurement, and every $S_{B}$ events during the background measurement, and if only integral numbers of registered events are recorded without regard to incompleted sets of $\mathrm{S}_{\mathrm{G}}$ or $\mathrm{S}_{\mathrm{B}}$ events, an additional source of variance is introduced. Two types of circuits are in common use: in the first type, any events arriving after the last completed register are ignored when the scaling circuit is reset and recorded, so that a measurement which is only 1 count below a completed set of $N+1$ registered sets of events is recorded as $\mathrm{N}$ registered sets of events; in the second type, the reading on the register is increased by $l$ if the next set of $S$ counts has been at least half completed. With the first type, a reading of $N$ registered sets of events implies a number of events in the counter anywhere between $N S$ and $(N+1)$ $\times S$ - 1; with the second type, a reading of $\mathrm{N}$ registered sets of events implies a number of events in the counter between $\left(N-\frac{1}{2}\right) S$ and $\left(N+\frac{1}{2}\right) S-1$. Any value in the range is equally likely in either case. The expected value of the number of events in the counter is obviously $\left(N+\frac{1}{2}\right) S-\frac{1}{2}$ in the first case and NS $-\frac{1}{2}$ in the second case; the distribution function in each case is seen to be a rectangular function S - I counts wide. For normalization, the height of the rectangle must be $\frac{1}{S-1}$, so that in the first case

$$
P_{x}(u)=\left\{\begin{aligned}
0, & u<N S \\
\frac{1}{S-1}, & N S \leq u \leq(N+1) S-1 . \\
0, & u>(N+1) S-1,
\end{aligned}\right.
$$

and in the second case,

$$
P_{x}(u)=\left\{\begin{aligned}
0, & u<\left(N-\frac{1}{2}\right) S \\
\frac{1}{S-1}, & \left(N-\frac{1}{2}\right) S \leq u \leq\left(N+\frac{1}{2}\right) S-1 \\
0, & u>\left(N+\frac{1}{2}\right) S-1 .
\end{aligned}\right.
$$

The variance of this distribution may be obtained from equation III. 33 for either case, 


$$
\begin{aligned}
\operatorname{var}(\mathrm{N})=\int_{\left(\mathrm{N}-\frac{1}{2}\right) S}^{\left(\mathrm{N}+\frac{1}{2}\right) \mathrm{S}-1}\left(\mathrm{x}-\mathrm{NS}+\frac{1}{2}\right)^{2} \frac{\mathrm{dx}}{(\mathrm{S}-1)} & =\int_{\mathrm{NS}}^{(\mathrm{N}+1) \mathrm{S}-1} \\
& \times\left(\mathrm{y}-\left(\mathrm{N}+\frac{1}{2}\right) \mathrm{S}+\frac{1}{2}\right)^{2} \frac{\mathrm{dy}}{(\mathrm{S}-1)} .
\end{aligned}
$$

The identity of the two integrals may be seen by a transformation of variables, $y=x+\frac{1}{2} S$. Upon performing the integration and simplifying, the result is

$$
\operatorname{var}(n)=\frac{(S-1)^{2}}{12}
$$

This variance must be added to each of the variances included in equation V. 5 for both gross and background measurements, so that in place of equation V.7 we have

$$
\operatorname{var}(z)=\frac{g}{{ }^{t_{G}}}+\frac{b}{t_{B}}+\frac{\left(s_{G}-1\right)^{2}}{12 t_{G}^{2}}+\frac{\left(s_{B}-1\right)^{2}}{12 t_{B}^{2}} .
$$

When high scaling factors are used, the variance arising from neglect of the "interpolation lights" between even registers can affect the computed variance quite appreciably.

A final term to be included in the estimate of the variance of each measurement depends on the counting equipment. It seems reasonable to assume that the equipment itself is subject to variation, for example in the positioning of the sample on consecutive measurements or in the level of the counter discriminator; it also seems reasonable to assume that the effect of such variation on the counting rate is proportional to the counting rate. We therefore postulate an additive variance for both gross and background counting rates, proportional to the square of the counting rate; the constant of proportionality will be dependent on the actual instrument and perhaps on the species counted.

Assuming for the moment that the constant of proportionality depends only on the instrument, we must realize that the sample positioning mechanism is part of the instrument for the gross count, but not for the background count; the constants for the two measurements will therefore be different. Estimation of such constants is extremely difficult, especially when they are small, since the only measurement that can be made also involves random variances of the types described above; a technique will be discussed later. 
If the variance due to counter variation is $\gamma^{2} g^{2}$ for the gross count and $\beta^{2} b^{2}$ for the background count rate, equation $\left.V 1\right]$ then becomes

$$
\operatorname{var}(z)=\frac{g}{t_{G}}+\frac{b}{t_{B}}+\frac{\left(S_{G}-1\right)^{2}}{12 t_{B}^{2}}+\frac{\left(S_{B}-1\right)^{2}}{12 t_{B}^{2}}+\gamma^{2} g^{2}+\beta^{2} b^{2}
$$

This expression is used to evaluate the proper weighting factors for use in a least-squares calculation of decay data, as described in Chapter IV.

A matrix inversion method is highly desirable, since the inverse matrix of the normal equations (see equations III.92d, 104f, 130, and $142 \mathrm{~g}$ )(multiplied by $\frac{x^{2}}{m-n}$ when this term is greater than unity) is the covariance matrix of the parameters, consequently, the diagonal elements of that matrix are the variances of the $x_{1}$ We then have an estimate of the precision of the amount of each component present, since the standard exior of $x_{1}$ is the square root of its variance.

If the variance due to counter variation is appreciable and is also dependent on the species being counted, proper weighting for a least-squares solution for a multicomponent system becomes very laborious. Estimation of the variance of a given measurement a priori is impossible, since the proportions of the various kinds of radiation making up the observed count are not known until after the resolution is done, if the counter variation constants for the various species are known from independent measurements on singlecomponent systems, the calculation may be done by successive approximations. Such a case may arise when the amplifying circuit of the counter has an energy cutoff which is subject to random drift, radiations producing pulses in the counter close to the cutoff level will be much more severely affected than those which produce pulses well removed from the cutoff. The practice of counting single photopeaks for decay, discriminating out pulses of both higher and lower energy than the gamma radiation of interest, is particularly subject to variation of this kind.

\section{Estimation of Variance Due to Instrumental Variation}

In order to make an estimate of the variance due to instrumental variation from a set of experimental data, it is necessary to resort to an iterative 
process. If the variance due to instrumental background is desired it can be obtained from a series of background measurements by the technique to be described below; in almost all cases it will betrivially small compared to the instrumental variation while counting an actual sample. In what follows we neglect it.

We resort to the $x^{2}$ test described in Chapter III, Section 5 (equations III. 138, 139, and 140) to give us an indication of the magnitude of instrumental variance. We perform a least-squares solution using equation $\mathrm{V} .12$ to give the weighting factors, assuming initially that the constant $\gamma$ is zero. If $\chi^{2}$ is significantly larger than its expected value, we repeat the solution using a trial value of $\gamma$ which is believed to be reasonable. The new value of $\chi^{2}$ will of necessity be smaller; if it is still too large, the trial value is increased and the calculation repeated again. Once a trial value of $\gamma$ is found which reduces $x^{2}$ below its expected value, a "best" value of $\gamma$ can be approached with a limited number of iterations by any one of a number of schemes of interpolation between trial values.

This system makes the obviously false assumption that the observed value of $x^{2}$ for this particular set of observations is precisely equal to its expected value; the "best value" of $\gamma$ found from any one set of data is relatively worthless. However, it seems reasonable to as sume that the observed $x^{2}$ values of a number of sets of good data, properly weighted, will be distributed about the expected value; consequently we expect that the set of calculated "best values" of $\gamma$ will be distributed about the true value. A large number of sets of observations should then give a number of values of $\gamma$ which, when averaged, should give a very reasonable estimate of $\gamma$.

Such a procedure involves a very large amount of sample preparation, counting, and especially calculation; if the experimental data to be processed are not of the highest precision the effort is probably not justified. The use of high speed computers for the iterative calculations is practically mandatory.

A more rapidly converging method can be derived as follows: Let

$$
\begin{aligned}
Y_{i} & =\text { the "true" value of the count rate at time } t_{i}, \\
\eta_{i} & =\text { the least-squares computed value of the rate at } t_{i}, \\
y_{i} & =\text { the observed value of the rate at } t_{i}, \\
r_{i} & =\text { the residual at } t_{i},
\end{aligned}
$$


that is,

$$
\mathrm{r}_{1}=\eta_{1}-\mathrm{y}_{1}
$$

where $\eta_{1}$ is computed without allowance made for instrumental variance.

Now to a good approximation,

$$
E\left(r_{1}^{2}\right) \approx E\left[\left(Y_{1}-y_{1}\right)^{2}\right]=\sigma_{1}^{2}+\gamma^{2} y_{1}^{2}
$$

so that

$$
E\left[\frac{r_{1}^{2}-\sigma_{1}^{2}}{y_{1}^{2}}\right] \approx \gamma^{2}
$$

If there are $m$ observations and $n$ components there are $m$ - $n$ degrees of freedom in the system. Therefore we expect that

$$
\frac{1}{m-n} \sum_{1}\left(\frac{r_{1}^{2}-\sigma_{1}^{2}}{y_{1}^{2}}\right) \approx \gamma^{2}
$$

In three cases selected at random an estimation of $\gamma$ by this method gave values which were within $2 \%$ (in each case) of the value obtained by the iteration method after a dozen iterations. The value so obtained can obviously be used to recompute the $\eta_{1}$ to permit an iterative improvement in $\gamma$.

4. Effect of Input Parameters on Resolution

We have considered the decay constants $\lambda_{1}$ of the system under consideration to be fixed and absolutely known up to this point. A detalled consideration of the determination and precision of the $\lambda_{1}$ must be deferred to the following chapter, it is, however, convenient to consider at this point the effect of a slight change in one of the $\lambda_{1}$ on the derived values of the system parameters $x_{1}$ and on the goodness of $f_{1}$ of the data to the calculated curve, as described by $x^{2}$.

The set of $\mathrm{n}$ normal equations for the system as described in Chapter III, equation III. $92 \mathrm{~d}$ with appropriate substitution, are

$$
\sum_{1=1}^{n}\left[\sum_{j=1}^{m} \frac{\left.e^{-\left(\lambda_{1}+\lambda_{k}\right) t}\right]}{\operatorname{var}\left(y_{j}\right)}\right] x_{1}=\sum_{j=1}^{m} \frac{y_{j} e^{-\lambda_{k} t}{ }_{j}}{\operatorname{var}\left(y_{j}\right)}
$$


for a set of $m$ observations on $n$ components, where $k$ has each value from $I$ to $n$. We desire to know the effect on each $x_{2}$ of a small variation in a particular $\lambda$, symbolized by $\lambda_{s}$, in other words, we want the quantity $\frac{\partial x_{1}}{\partial \lambda_{s}}$ for each 1 and for each $s$.

If one performs the operation of partial differentiation with respect to $\lambda_{s}$ on the set of equations V.J7, one obtains the set of equations $(1 \leq k \leq n)$ $\sum_{1=1}^{n}\left[\sum_{j=1}^{m} \frac{e^{-\left(\lambda_{1}+\lambda_{k}\right) t} j}{\operatorname{var}\left(y_{j}\right)}\right] \frac{\partial x_{1}}{\partial \lambda_{s}}-\sum_{1=1}^{n}\left[x_{1} \sum_{j=1}^{m} \frac{t_{j}\left(\delta_{1 s}+\delta_{k s}\right) e^{-\left(\lambda_{1}+\lambda_{k}\right) t} j}{\operatorname{var}\left(y_{j}\right)}\right]$

$$
=-\sum_{j=1}^{m} \frac{\mathrm{t}_{j} \delta \mathrm{s}^{\mathrm{y}} \mathrm{j}^{-\lambda_{\mathrm{k}} \mathrm{t}} \mathrm{var}(\mathrm{y})}{\mathrm{v})}
$$

where

$$
\begin{aligned}
\delta_{1 \mathrm{~J}} & =0, \quad 1 \neq 3 \\
& =1, \quad 1=3,
\end{aligned}
$$

which upon rearrangement gives

$$
\begin{array}{r}
\sum_{1=1}^{n}\left[\sum_{j=1}^{m} \frac{e^{-\left(\lambda_{1}+\lambda_{k}\right) t} j}{\operatorname{var}\left(y_{j}\right)}\right] \frac{\partial x_{1}}{\partial \lambda_{s}}=\sum_{i=1}^{n}\left[x_{1}\left(\delta_{1 s}+\delta_{k s}\right) \sum_{j=1}^{m} \frac{t_{j} e^{-\left(\lambda_{1}+\lambda_{k}\right) t} j}{\operatorname{var}\left(y_{j}\right)}\right] \\
-\delta_{k s} \sum_{j=1}^{m} \frac{y_{j} e_{j}^{-\lambda_{k}{ }^{t} j}}{\operatorname{var}\left(y_{j}\right)}
\end{array}
$$

for any value of $l \leq s \leq n$ If this set of equations is expressed in matrix form it will be seen at once that the matrix of the coefficients of the new set of unknowns $\partial x_{1} / \partial \lambda_{s}$ is identical with the matrix of the normal equations, if these have already been solved by matrix inversion, the inverse matrix is already avalable. The values of the $x_{1}$ from the solution of the normal equations may be inserted into the right members of the set of equations to give the components of a vector $v_{s}$ which, multiplied from the left by the inverse matrix of the normal equations, yields the vector whose components are $\partial x_{2} / \partial \lambda_{s}$. If a matrix $P$ is formed whose columns are the set of vertors $v_{s}$ with $s$ taking on the values $l$ to $n$, in turn, then this matrix multiplied 
from the left by the inverse matrix of the normal equations will give a new matrix $Q$ such that

$$
Q_{i s}=\frac{\partial x_{i}}{\partial \lambda_{s}}
$$

which is a compact solution to our problem.

It is perhaps more convenient to express the components of $Q$ in terms of fractional changes and to convert $Q$ to a matrix $Q^{\prime}$ such that

$$
Q^{\prime}{ }_{i s}=-\frac{\lambda_{s}}{x_{i}} \frac{\partial x_{i}}{\partial \lambda_{s}}=\frac{T_{s}}{x_{i}} \frac{\partial x_{i}}{\partial T_{s}}
$$

where

$$
\mathrm{T}_{\mathrm{s}}=\frac{\ln 2}{\lambda_{\mathrm{s}}} \text {. }
$$

The negative sign is inserted to put the significance of the number so derived in terms of an alteration in the half-life value, rather than the decay constant, since most workers are used to thinking in terms of half-lives rather than decay constants.

We can now derive the effect of varying an input $\lambda$ on the fit of the data to the calculated curve. We have

$$
x^{2}=\sum_{j=1}^{m} \frac{r_{j}^{2}}{\operatorname{var}\left(y_{j}\right)}=\sum_{j=1}^{m} \frac{\left(y_{j}-\sum_{i=1}^{n} x_{i} e^{-\lambda t_{j}}\right)^{2}}{\operatorname{var}\left(y_{j}\right)} .
$$

Therefore

$\frac{\partial\left(x^{2}\right)}{\partial \lambda_{s}}=\sum_{j=1}^{m} \frac{2\left(y_{j}-\sum_{i=1}^{n} x_{i} e^{-\lambda i_{i}^{t} j}\right)\left[t_{j} x_{s} e^{-\lambda s^{t} j}-\sum_{i=1}^{n} e^{-\lambda i j}\left(\frac{\partial x_{i}}{\partial \lambda_{s}}\right)\right]}{\operatorname{var}\left(y_{j}\right)}$.

From the least-squares solution we get the values of the $x_{i}$ and from the matrix $Q$ described above we get the values of the $\partial x_{i} / \partial \lambda_{s}$. A positive value of this derivative means $\lambda_{s}$ should be reduced (i. e., the half-life should be 
increased) to improve the $f_{1 t}$, a negative value means the reverse. This calculation can be made the basis of an iterative method for improving the values of the input $\lambda$, providing that fairly good values are available inıtially.

\section{Criteria for the Rejection of Data}

It frequently happens that a set of data contains one or more points which do not actually belong to the set, due either to an instrument malfunction or to human error on the part of the experimenter. Typical errors are mis reading the instrument, counting the wrong sample, counting the sample in the wrong counting position, falling to remove or insert an absorber, falling to remove a previous sample, falling to set proper discriminator levels, falling to record the data in legible form, resulting in misreading of the written data, and so on ad nauseam. If the instrument (or the operator) is so erratic that many points are subject to such nonstatistical errors, the experiment is worthless, there is no way of selecting the good data from the total set a priori. If no further information is available, the experiment should be redesigned. If the fault is in the operator, suitable corrective action sometimes requires considerable ingenuity.

If the set of data contains only one or two erroneous points, these can usually be detected by inspection of a graphical presentation of sample activity plotted against time. This is, however, a subjective method and takes no account of the precision of the individual measurements. An objective criterion for rejection of suspected data is desirable

For convenience in evaluating data, it is highly desirable to tabulate the points in order of time, with their residuals expressed both as "percentage error" and as "number of standard deviations," 1.e., the weighted residual. Whenever the magnitude of the weighted residual exceeds about 3 , the point should be examined further, since such a large deviation is highly unlikely to occur through random error. If the weighted residual is unreasonably large, but the percentage error is quite small (as often happens when a very large number of events is recorded) then no appreciable improvement in the answer is achieved by rejecting the point in question.

One is then led to the idea that a point should be rejected if: (1) The point is separated from the "true" calculated curve by more than about three of its standard deviations, and (2) Omission of the point in question alters 
the final answer appreciably. The "true" calculated curve may be estimated from the curve calculated without the suspected point. A systematic check of every point in a set of $\mathrm{m}$ points, then, will require a series of $m+1$ leastsquares calculations - a very laborious task.

It is possible to approximate the same criterion by some far simpler tests. One can estimate that a human error will cause a percentage deviation of the observed point greater than a certain arbitrarily selected level, say $5 \%$; or alternatively one can decide that rejection of a single point in the set which is within 5\% of the computed curve (using all points) will not affect the answer appreciably. One can then class all points with a percentage deviation from the calculated curve of $5 \%$ or greater, and a weighted residual of 3 or greater, as "suspect."

Unfortunately, a simple scheme which rejects all such points, or even rejects them one at a time starting with the point with the largest deviation, proves in practice to have serious flaws - in fact, it retains bad points and rejects good ones a fair fraction of the time. An additional criterion which has been found to give good results in our laboratory is based on the following approach: assume the existence of a set of "good" data points with a calculated curve passing through them. The deviations of the points from the curve will be distributed, some positive and some negative, none very large, and more or less at random. If now a "bad" point is added to the data and the curve is recomputed, the presence of the bad point will tend to displace the new computed curve toward that point and away from the good points on either side. The new curve will then pass between the good points and the bad one in the region of the bad point. This gives us our additional criterion: for automatic rejection, the deviation of the suspect point (suspect in the sense described above) from the calculated curve must be in the opposite sense from the deviations of the points on either side of it; or if the suspect point is the first or last point, its deviation must be in the opposite sense from that of the next point to it.

This triple criterion has been in use in Livermore for some time and has been applied to literally hundreds of sets of data. It has occasionally caused unneeded rejection of a good point, usually when the bad point is the second data point - in this case the first point is sometimes rejected also but if data are sufficiently redundant this usually does no harm. 
If there are suspect points in the data, and if the third criterion for rejection is not met, one should examine the fit of the data to the calculated curve. Often a systematic pattern will show up in the residuals, most easily seen as a groupıng of positive and negative residuals in clusters. This grouping indicates an improper form for the function being fitted - the sample is impure, or the wrong half-lives are being used.

If the analysis of the data is performed by a programmed computer, it is very desirable to obtain an output which presents the input parameters, the results, a tabulation of points and their deviations, and the results of the $x^{2}$ test. A common complaint of those using least-squares analysis in an improper manner is that any function can be fitted to ary set of data by a leastsquares method, while this is undoubtedly true, it is easy to avoid gross errors if one considers not only the answers so obtained but also their precision, the goodness of fit as given by $x^{2}$, and the pattern shown by the residuals. Also, providing that suitable computing facilities are available, a great deal of labor may be saved and a great many experiments protected to some degree against subjective bias.

\section{Avosdance of Computer Overflow}

It orcasionally happens that a multicomponent system has in it a shortlived daughter of a longer-lived parent, and that the purification of the parent species is performed some time after the time of interest. If the activity measurement is carried out in such a way that the daugher component must be included in the resolution, 1 .e, if the growth of the daughter is observed, difficulties may arise in the analysis if precautions are not taken. The time elapsed between zero-time and the time of measurement may be, and often is, so large with respect to the daughter half-life that an attempt to evaluate the necessary exponential functions will result in a computer overflow. This problem can always be avoided by a substitution of a spurious cero-time, conveniently taken as the time of the first count, the resolution can be performed to give results as of that time and these results extrapolated back to the true zero-time. No difference will be obtained in the answers by this method. The advantage gained is that a separate exponential extrapolation factor is used for each component, the exponent can be evaluated and tested for magnitude before exponentiation, and one can refrain from extrapolating those components whose exponents are too large. Such coefficients are without physical meaning in any case. 
Since extrapolation involves merely multiplying by a constant, the fractional precisions of the $x_{1}$ are not changed by this process. However, if one is interested in the sensitivity of the $x_{1}$ to the input half-lives, the $Q-$ matrix (see equation $V-20$ ) as derived by this method will be changed by extrapolation. Let

$$
y_{1}=x_{1} e^{\lambda_{1} \Delta t}
$$

where $\Delta t$ is the time between the true zero-time and the time of the first count. Then

$$
\frac{\partial y_{1}}{\partial \lambda_{j}}=\frac{\partial x_{1}}{\partial \lambda_{j}} e^{\lambda_{1} \Delta t}+\delta_{1 j}(\Delta t) x_{1} e^{\lambda_{1} \Delta t}
$$

where $\delta_{1 \mathrm{j}}$ has the usual meaning.

\section{Analysis of Replicate Samples, Analysis of Variance}

It is the usual practice to do replicate analyses on radiochemical samples whenever possible. A set of replicates will, in general, give a set of approximately equal answers, since the result of a radiochemical analysis is subject to statistical imprecision, we would not expect replicate samples to give identical answers except fortuitously. We must bear in mind also that the statistical imprecision of a single radiochemical analysis, as estimated from the least-squares solution, does not give a true picture of the precision of the result, there are analytical variations possible which have a systematic, rather than a random, effect on all measurements performed on a single sample. Examples are an error in the measurement of the aliquot taken for analysis, an error in the measurement of the recovered yield, $3 n$ inhomogeneity in the mounted counting sample, resulting in a systematic perturbation in the counting rate, an error in the amount of carrier added, and so on. We assume that these analytical variations have a random effect on the answers from a set of replicate samples. It remains to develop a technique for estimating the imprecision due to such variations from the avallable data. It should also be noted that there are numerous systematic errors which can distort the results of an entire set of replicates. Llimination of such errors is a matter of careful expermental technique, no amount of data manipulation can exther detect or compensate for them.

Each replicate answer may be considered to be the expected value of a probability distribution function of known varıance. These expected values 
do not all coincide. Furthermore, since these distribution functions are not all of equal variance, their worths in establishing an overall "best" value are not all equal. We desire to analyze the results of such replicate analyses mathematically in order to make an estimate of the imprecision due to analytical technique. When this is available, one can then add the variance due to analytical technique to the variance due to statistical fluctuations in the decay data to get the total variance for each replicate; the reciprocal of this total variance is then used as a weighting factor to average the replicate results, and their variances, as before to give a best estimate of the true value for the experiment and its variance.

The problem is analogous to one discussed by Davies 9 in which a number of analyses $n_{i}$ is performed on each of $k$ samples, the variance of an individual analysis is $\sigma_{0}^{2}$ and the variance between samples is $\sigma_{1}{ }^{2}$. The numbers $n_{i}$ are not necessarily equal between samples. It can be shown that, if $x_{i j}$ represents the result from the jth replicate analysis on the ith sample, the quantity

$$
M_{1}=\frac{1}{k-1}\left\{\sum_{i=1}^{k} \frac{\left(\sum_{j=1}^{n_{i}} x_{i j}\right)^{2}}{n_{i}}-\frac{\left(\sum_{i=1}^{k} \sum_{j=1}^{n_{i}} x_{i j}\right)^{2}}{\sum_{i=1}^{k} n_{i}}\right\}
$$

is an estimate of $\sigma_{0}^{2}+\bar{n} \sigma_{1}^{2}$, where

$$
\bar{n}=\frac{\left(\sum_{i=1}^{k} n_{i}\right)^{2}-\sum_{i=1}^{k} n_{i}^{2}}{(k-1) \sum_{i=1}^{k} n_{i}} .
$$

Consequently, an estimate of $\sigma_{l}^{2}$ is given by

$$
\sigma_{1}^{2}=\frac{M_{1}-\sigma_{0}^{2}}{\bar{n}} .
$$

${ }^{9}$ Owen L. Davies, Editor, Statistical Methods in Research and Production (Hafner Publishing Co., N. Y., 1958), III Ed. (revised), pp. 134-136. 
In order to apply this result to our problem, we consider our various results $x_{1}$ to be averages of undetermined numbers $n_{1}$ of analyses each with a variance $\sigma_{0}^{2}$, from Chapter III, equation III.51, $\operatorname{var}(\mathrm{x})$ is given by

$$
\operatorname{var}\left(\mathrm{x}_{1}\right)=\frac{1}{\mathrm{n}_{1}} \cdot \sigma_{0}^{2}
$$

and consequeritly

$$
\mathrm{n}_{1}=\sigma_{0}^{2} \cdot \frac{1}{\operatorname{var}\left(\mathrm{x}_{1}\right)} .
$$

Since we have assumed that

$$
x_{1}=\frac{\sum_{j=1}^{n_{1}} x_{1 j}}{n_{1}}
$$

then

$$
\sum_{j=1}^{n_{1}} x_{1 j}=n_{1} x_{1}=\frac{x_{1}}{\operatorname{var}\left(x_{1}\right)} \cdot \sigma_{0}^{2} .
$$

Consequently,

$$
M_{1}=\frac{\sigma_{0}^{2}}{k-1}\left\{\sum_{1=1}^{k} \frac{x_{1}^{2}}{\operatorname{var}\left(x_{1}\right)}-\frac{\left[\sum_{1=1}^{k} \frac{x_{1}}{\operatorname{var}\left(x_{1}\right)}\right]^{2}}{\sum_{1=1}^{k} \frac{1}{\operatorname{var}\left(x_{1}\right)}}\right\}
$$

and

$$
\overline{\mathrm{n}}=\frac{\sigma_{0}^{2}}{\mathrm{k}-1}\left\{\frac{\left[\sum_{1=1}^{\mathrm{k}} \frac{1}{\operatorname{var}\left(\mathrm{x}_{1}\right)}\right]^{2}-\sum_{1=1}^{\mathrm{k}} \frac{1}{\left[\operatorname{var}\left(\mathrm{x}_{1}\right)\right]^{2}}}{\sum_{1=1}^{\mathrm{k}} \frac{1}{\operatorname{var}\left(\mathrm{x}_{1}\right)}}\right\}
$$

and therefore, substituting in V.28 and simplifying, 
$\sigma_{1}^{2}=\frac{\left[\sum_{1=1}^{k} \frac{1}{\operatorname{var}\left(x_{1}\right)}\right]\left[\sum_{1=1}^{k} \frac{x_{1}^{2}}{\operatorname{var}\left(x_{1}\right)}\right]-\left[\sum_{1=1}^{k} \frac{x_{1}}{\operatorname{var}\left(x_{1}\right)}\right]^{2}-(k-1) \sum_{1=1}^{k} \frac{1}{\operatorname{var}\left(x_{1}\right)}}{\left[\sum_{1=1}^{k} \frac{1}{\operatorname{var}\left(x_{1}\right)}\right]^{2}-\sum_{1=1}^{k} \frac{1}{\left[\operatorname{var}\left(x_{1}\right)\right]^{2}}}$.

Note that $\operatorname{var}\left(x_{1}\right)$ as used above refers only to the variance of the sample due to fluctuations in the decay data, in order to average the replicate values as was done in Chapter III, equations III. 77 and 78 , the estimate for $\sigma_{1}^{2}$ should be added to each variance, so that for the best average value of $k$ replicates,

$$
\bar{x}=\frac{\sum_{1=1}^{k} \frac{x_{1}}{\sum_{1=1}^{k} \frac{1}{\operatorname{var}\left(x_{1}\right)+\sigma_{1}^{2}}}}{\operatorname{var}\left(x_{1}\right)+\sigma_{1}^{2}}
$$

and

$$
\operatorname{var}(\bar{x})=\frac{1}{\sum_{1=1}^{k} \frac{1}{\operatorname{var}\left(x_{1}\right)+\sigma_{1}^{2}}}
$$

A derivation of equation V.35 follows:

Let the results of our replicate analyses be given by $x_{1}$, with each of which is associated a variance var $\left(x_{1}\right)$ derived exclusively from counting statistics. $\operatorname{Var}\left(\mathrm{x}_{1}\right)$ is then obtained from the least-squares resolution of the decay curve of the $1^{\text {th }}$ sample. We as sume that each $x_{1}$ is equivalent to an average of $n_{1}$ virtual individual results, each with the same variance $\sigma_{0}^{2}$.

Given any pair of virtual measurements, $x_{1 j}$ and $x_{\ell m}$ ' let their mean be represented by $\mu_{1 \jmath \ell m}$, then an estimate of the variances $\sigma_{0}^{2}$ and $\sigma_{1}^{2}$ may be obtained from this pair of measurements as follows.

If $1=\ell$, then $\sigma_{1}^{2}$ is not estimated, since the two samples are from the same group.

If $1=\ell$ and $\mathrm{J}=\mathrm{m}$, no variance is estimated. 
Let the variance estimated from our pair be

$$
V_{1 j \ell m}=\frac{\left(x_{1 j}-\mu_{1 j \ell m}\right)^{2}+\left(x_{\ell m}-\mu_{1 j \ell m}\right)^{2}}{2-1},
$$

and

$$
\mathrm{V}_{1 \mathrm{~J} \ell \mathrm{m}} \approx \sigma_{0}^{2}\left(1-\delta_{1 \ell} \cdot \delta_{\mathrm{jm}}\right)+\sigma_{1}^{2}\left(1-\delta_{1 \ell}\right)
$$

Substituting in V 39 for $V_{1 j \ell m}$, given that

$$
\mu_{1 \mathrm{~J} \ell \mathrm{m}}=\frac{\mathrm{x}_{1 \mathrm{j}}+\mathrm{x}_{\ell \mathrm{m}}}{2}
$$

we get

$$
\frac{1}{2}\left(\mathrm{x}_{1 \mathrm{j}}-\mathrm{x}_{\ell \mathrm{m}}\right)^{2} \approx\left(1-\delta_{1 j} \delta_{\ell \mathrm{m}}\right) \sigma_{0}^{2}+\left(1 \delta_{1 \mathrm{j}}\right) \sigma_{1}^{2}
$$

Summing over all possible pairs to get the best estimate, we get

$$
\begin{aligned}
& \sum_{1=1}^{\mathrm{k}} \sum_{\mathrm{j}=1}^{\mathrm{n}_{\mathrm{l}}} \sum_{\ell=1}^{\mathrm{k}} \sum_{\mathrm{m}=1}^{\mathrm{n}_{\ell}}\left(\frac{\mathrm{x}_{1 \mathrm{j}}^{2}+\mathrm{x}_{\ell \mathrm{m}}^{2}}{2}-\mathrm{x}_{1 \mathrm{j}} \mathrm{x}_{\ell \mathrm{m}}\right) \approx \sigma_{0}^{2} \sum_{1}^{\mathrm{k}} \sum_{j}^{\mathrm{n}_{1}} \sum_{\ell}^{\mathrm{k}} \sum_{\mathrm{m}}^{\mathrm{n}}\left(1-\delta_{1 \mathrm{j}} \delta_{\ell \mathrm{m}}\right) \\
& +\sigma_{1}^{2} \sum_{1}^{\mathrm{k}} \sum_{\mathrm{j}}^{\mathrm{n}_{1}} \sum_{\ell}^{\mathrm{k}} \sum_{\mathrm{m}}^{\mathrm{n} \ell}\left(1-\delta_{1 \mathrm{j}}\right)
\end{aligned}
$$
gives

$$
N \sum_{1}^{k} \sum_{j}^{n_{1}}\left(x_{1 j}^{2}\right)-\left(\sum_{1}^{k} \sum_{j}^{n_{1}} x_{1 j}\right)^{2} \approx \sigma_{0}^{2}\left(N^{2}-N\right)+\sigma_{1}^{2}\left(N^{2}-\sum_{1=1}^{k} n_{1}^{2}\right)
$$

Now $\sigma_{0}^{2}$ may be estimated within each sample by

$$
\sigma_{0}^{2} \approx \frac{\sum_{j=1}^{n_{1}}\left(x_{1 j}-x_{1}\right)^{2}}{n_{1}-1},
$$


so that

$$
\sum_{j=1}^{n_{1}}\left(x_{1 j}-x_{1}\right)^{2} \approx\left(n_{1}-1\right) \sigma_{0}^{2}
$$

Again summing over all samples to obtain the best estimate, we get

$$
\sum_{1=1}^{k} \sum_{j=1}^{n_{1}}\left(x_{1 j}^{2}\right)-\sum_{1=1}^{k} n_{1} x_{1}^{2} \approx(N-k) \sigma_{0}^{2}
$$

which gives

$$
\sum_{i=1}^{k} \sum_{j=1}^{n}\left(x_{1 j}^{2}\right) \approx \sum_{1=1}^{k} n_{1} x_{1}^{2}+(N-k) \sigma_{0}^{2} .
$$

Substituting this result into V.43 and rearranging, we get

$$
\sigma_{1}^{2}=\frac{N \sum_{1}^{k} n_{1} x_{1}^{2}-\left(\sum_{1}^{k} n_{1} x_{1}\right)^{2}-N(k-1) \sigma_{0}^{2}}{N^{2}-\sum_{1}^{k} n_{1}^{2}} .
$$

If we now insert our definition of $N$ and substitute for $n_{1}$ from equation V.30, upon simplification we get the desired result, equation V.35. It will be observed that the value of $\sigma_{0}^{2} 1$ is indeterminate, as it must be since it represents the variance of a distribution of virtual objects postulated for convenience.

A negative value of $\sigma_{1}^{2}$ signifies that, fortuitously, the replicate measurements agree better than one would expect from their precision This occurrence is analogous to a value of count varıance less than its expected value, as discussed on page 56, such a result merely implies that there is no clear evidence for analytical variation, and therefore $\sigma_{1}{ }^{2}$ should be given the value zero in Equations V.36 and V.37. Frequent appearance of negative values of $\sigma_{1}^{2}$ in a set of results indicates that errors of the input numbers are being improperly assigned. 


\section{THE IDENTIFICATION OF COMPONENTS AND MEASUREMENT OF HALF-LIVES OR DECAY CONSTANTS}

\section{Nature of the Problem}

We have been operating on the assumption that the decay constants of the components of our systems have been precisely known While it is true that the decay constant of a nuclear species is not a variate, in the sense that it is not subject to variation due to sampling, nevertheless it must be realized that the only method of evaluating a decay constant is by observing the process of decay - which inevitably involves random statistical errors. In this sense, then, a decay constant must be treated as a variate, although its value must be considered fixed during any one resolution calculation - variation in $\lambda$ makes a systematic, rather than a random, variation in the results of a resolution. In this chapter we shall consider several different approaches to the measurement of $\lambda$ or its equivalent, the measurement of half-life, from a set of data, together with the inseparable problem of the detection of the number of components in the system.

It is of the utmost importance in measuring the half-life of a species to make sure that the sample under examination is free of even the smallest amount of radioactive impurities. A grossly impure sample is often easy to identify, if the species involved are reasonably short-lived, the decay curve will clearly show the presence of more than one component unless the halflife of the impurity is similar to that of the species of interest If, however, the extent of contamination is slight, or the species involved are too longlived to decay to any great extent in a reasonable period, detection of an impurity is more difficult. It can sometimes be accomplished by analysis of the radiations from the sample, sometimes the only recourse is to observe the specific activity of the sample as it is subjected to more and more rigorous chemical purification. If the impurity is isotopic, it is necessary either to use an isotope separator to prepare a pure sample, or to estimate the desired half-life by a treatment of the data which takes account of the presence of components other than the one of interest

An example of the effect of an impurity on the measurement of a halflife can be found in the early measurements of the half-life of Cdl15 formed from fission. The original reports on this nuclide assigned it a half-iffe of 
58 hours, when the existence of the isomeric state, with a half-life of 43 days, was discovered, re-examination of the data resulted in a change to the current best value for $\mathrm{Cd}^{115}$ of 53 hours - a change of nearly $10 \%$.

\section{Single-Component System of Short or Moderate Half-Life}

For simplicity, let us first consider a system which we are sure contains only one component during the entire period covered by the data. The decay of this system is given by

$$
\mathrm{n}=\mathrm{n}_{0} \mathrm{e}^{-\lambda \mathrm{t}}
$$

which can be transformed into linear form by taking logarithms:

$$
\ln \mathrm{n}=\ln \mathrm{n}_{0}-\lambda \mathrm{t} .
$$

If $\mathrm{n}$ is the observed counting rate, we may evaluate $\lambda$ graphically by plotting $\ln n$ against $t$ and measuring the slope of the line, if semilogarithmic paper is used, we may measure half-life as the time interval required for $n$ (not $\ln n$ ) to decrease by a factor of 2 .

A more precise and objective result is desirable If a redundant amount of data is available (1. e., more than two points) a least-squares solution can be performed as described in Chapter III, Section 5, using the set of equations VI.2 (one for each measured point). In order to weight the points properly we need the variance of $\ln \mathrm{n}$, if the data are fairly precise this may be obtained approximately as follows

$$
\begin{aligned}
& \operatorname{var}(\ln n)=\operatorname{var}\left\{\ln \left[E(n)+r_{1}-E(n)\right]\right\}, \\
& \operatorname{var}(\ln n)=\operatorname{var}\left(\ln \left\{E(n)\left[1+\frac{n-E(n)}{E(n)}\right]\right\}\right), \\
& \operatorname{var}(\ln n)=\operatorname{var}\left\{\ln E(n)+\ln \left[1+\frac{n-E(n)}{E(n)}\right]\right\}, \\
& \operatorname{var}(\ln n)=\operatorname{var}\left\{\ln \left[1+\frac{n-E(n)}{E(n)}\right]\right\} .
\end{aligned}
$$

Since $\frac{n-E(n)}{E(n)}$ is as sumed to be small, we may expand the logarithm as a series and neglect all powers of $\frac{n-E(n)}{E(n)}$ above the first, so that 


$$
\begin{aligned}
& \operatorname{var}(\ln n) \approx \operatorname{var}\left[\frac{n-E(n)}{E(n)}\right], \\
& \operatorname{var}(\ln n) \approx \operatorname{var}\left[\frac{n}{E(n)}-1\right]=\operatorname{var}\left[\frac{n}{E(n)}\right], \\
& \operatorname{var}(\ln n) \approx \frac{\operatorname{var}(n)}{[E(n)]^{2}} .
\end{aligned}
$$

We then have the proper weighting factors to permit a least-squares analysis of the data for the parameters $1 \mathrm{n} \mathrm{n}_{0}$ and $\lambda$. If a matrix solution is used we have an objective evaluation of var $(\lambda)$, as described in Chapter III, equations III.92d, J04f, 130, and J42g, it is the proper diagonal element of the inverse matrix, multiplied by $x^{2 /(m-2)}$ where this factor is greater than unity.

\section{Single-Component System with Long Half-Life}

If the half-life of the species under examination is so long that an experiment to observe the decay of a sample is difficult, another method must be used. There are three methods in general use. The simplest method depends on a direct measurement of specific activity, the second and more elaborate method depends on a careful comparison of the decay of the unknown species with that of a species of precisely known half-life, the third method depends on an internally calibrated method of observing decay over long periods of time.

Measurement of half-life by specific activity depends on the differential form of the fundamental law of radioactivity (equation II.1) which is

$$
\frac{\mathrm{dn}}{\mathrm{dt}}=-\lambda \mathrm{n}
$$

where $n$ is the number of atoms present and - dn/dt is the disintegration rate. Direct application of this formulation of the law requires two difficult measurements. an absolute measurement of the disintegration rate of the sample, corrected for solid angle, counter efficiency, and sample absorption and scattering effects, and a measurement of the number of atoms in the sample. With the improvement in absolute counting techniques, and the increasing sensitivity of analytical instrumentation such as the mass spectrometer for measurement of $\mathrm{n}$, this method is becoming more precise, but still requires great care. An example of the method, which gives some indications 
of the care which must be used, will be found in Fleming, Ghiorso, and Cunningham's article ${ }^{10}$ on the half-iıfe of uranium A similar measurement was carried out at Harwell ${ }^{11}$ to give the half-Iife of Cs 33 .

A technique which has been used by several workers 11,12 depends on the fact that two ionization chambers carefully made to be mechanically as nearly identical as possible will have nearly identical responses. A sample whose half-life is to be determined is placed in one chamber, and a very longlived standard sample in the other. The two chambers are then connected to a sensitive current measuring device, e.g, an electrometer tube, in such a way that the difference in current between the two chambers may be measured This difference is balanced to zero at the time $t_{0}$ and its change with time is noted. It will be seen that the "difference current" $I_{d}$ will have the form

$$
I_{d}=I_{0}\left[1-e^{-\lambda\left(t-t_{0}\right)}\right] \text {. }
$$

If the experimental sample is removed from its chamber, the "difference current" observed with the standard sample compared to an empty chamber will be a measure of $I_{0}$. We then have

$$
\ln \left(1-\frac{I_{d}}{I_{0}}\right)=-\lambda\left(t-t_{0}\right)
$$

In practice, in particular if the decay of the sample is appreciably rapid, the exact time $t_{0}$ may be inconvenient to obtain, one may equally well select an arbitrary time $t_{a}$ (near the time at which the chambers are exactly balanced, for convenience). Then let

$$
\begin{aligned}
& X=\ln \left(1-\frac{I_{d}}{I_{0}}\right)=-\lambda\left(t-t_{a}+t_{a}-t_{0}\right), \\
& X=\lambda\left(t_{a}-t_{0}\right)-\lambda\left(t-t_{a}\right) .
\end{aligned}
$$

10 E. H Fleming, Jr., A Ghiorso, and B B Cunningham, Phys Rev. 88, 642 (1952).

] F. Brown, G R. Hall, and D. N Walter, J Inorg Nucl Chem. 1 , 24] (1955).

12 E. Segrè and C E. Wregand, Phys. Rev. 75, 39 (1949). 
We may plot $X$ against $\left(t-t_{a}\right)$ and obtain $\lambda$ from the slope of the line, or for greater precision evaluate that slope by a least-squares method. Tobailem ${ }^{13}$ obtained the half-life of $\mathrm{Co}^{60}$ ( 5.27 years) to $] .3 \%$ in a series of measurements lasting only 32 days, and the half-Iife of $\mathrm{Ac}^{227}$ (21.6 years) to $2 \%$ in a series of measurements lasting only 137 days

If the standard sample decays appreciably during the course of the measurement, and if its half-life is precisely known, then one inserts $I_{0} e^{-\lambda s^{\left(t-t_{0}\right)}}$ into equation VI.7 in place of $I_{0}$. In this case it is necessary to have a good estimate for $t_{0}$, this necessity becomes less urgent as the halflife of the standard becomes larger.

A self-calibrating method for measuring long-lived decays has been applied to the measurement of the half-life of $\mathrm{Kr}^{85}$ (ref. 14) and of $\mathrm{Cs} 137$ (ref 15). The change in the ratio of $\mathrm{Kr}^{85} / \mathrm{Kr}^{86}$ in a gas sample, or $\mathrm{Cs}^{137} / \mathrm{Cs}^{135}$ in a fission-product cesium sample, can be followed for long times, since the fact that a ratio is measured is insurance against instrumental drift

\section{Multicomponent Systems - Detection}

Given a sample or set of data about which no information (other than statistical reliability) is avalabie, the first question which comes to mind is; 1s the system a single-component system, or is it not?

The simplest approach is to plot the data on semilogarithmic paper, $\log (\mathrm{n})$ vs $t$ as before, multicomponent systems will usually give curved lines if the data are well enough distributed in time. If, however, the data do not cover at least two or three half-lives of the apparent principal component, an erroneuus conclusion will often be drawn. (See Fig. VI.1.)

A more sensitive test may be applied by performing a least-squares analysis on the data, using the apparent half-life, if two components are actually present, a systematic pattern of deviations will appear, and the $x^{2}$ test will indicate a poor fit.

13 J Toballem, J Phys. Radium 16, 48 (1955).

${ }^{14} \mathrm{R} N$ Wonless and H. G. Thode, Can. J. Phys. 31, 517 (1953).

${ }^{15}$ C. G. Campbell, private communication, cited in F. Brown and G. R. Hall, Nuovo Cimento Suppl. (Ser. 10) 6 , 283 (1957). 


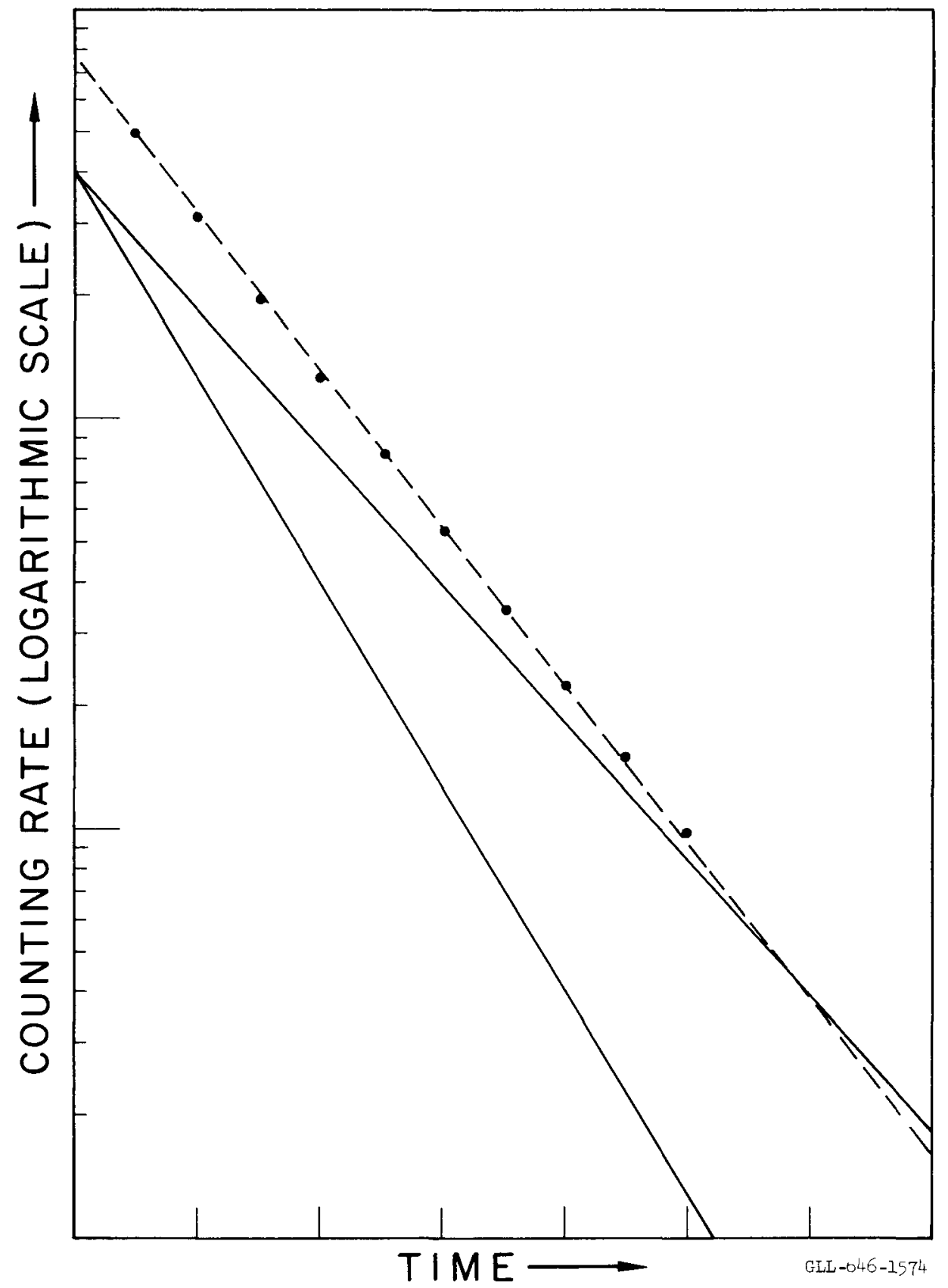

Fig. VI.l. Decay curve of a two-component system, with equal amounts of two species whose half-lives differ by a factor of 1.5. Deviation from a singlecomponent decay curve of intermediate half-life (dashed curve) is barely perceptible. This illustrates the difficulty of resolving two species with similar half-lives. 
A still more sensitive method for detecting the presence of more than one species is based on differential counting If the gamma spectrum of the sample is followed as a function of time, its shape may often be seen to change with time, a simpler way of accomplishing the same objective is to follow the decay of the sample on both beta and gamma detectors or through various thicknesses of absorbing material If the sample contains more than one component, the counting rates under different conditions will not all show the same time-dependence, indeed, if the counting conditions can be made sufficiently selective, it is sometimes possible to observe the decay of one or more species essentially pure. In this fortunate case, a good estimate of the half-lives of such species can be obtained. In any case, if the presence of more than cne component is suspected, counting should be continued until the activity of the sample has decayed to a small fraction of the original activity if possible

When there are two or more long-lived components it is not practical to follow the decay of the sample through many half-lives, if the components are not isotopic it is frequently possible to distinguish between them by careful chemical separations. If they are isotopic, the use of a mass-separator should be considered.

\section{Known Multicomponent Systems - Evaluation of $\lambda$}

If we are dealing with a system which is known to contain certain components, and only those components, the evaluation of the $\lambda$ is possible by several different methods. One usually has approximate values for the $\lambda$, which may be obtained graphically as described in Chapter IL, Section 2 these approximate values may then be improved by calculation. A direct solution in the manner of Chapter III is not possible, since the forms of the equations are not linear if the $\lambda$ are treated as unknowns, if, however, $\Lambda_{1}$ (the true $\lambda_{1}$ ) are considered to differ from the approximate $\lambda_{1}$ by a small amount $\epsilon_{1}$ so that

$$
\Lambda_{1}=\lambda_{1}+\epsilon_{1}
$$

then we can say that

$$
e^{-\Lambda_{1} t_{j}}=e^{-\left(\lambda_{1} t_{j}+\epsilon_{1} t_{J}\right)}=e^{-\lambda_{1} t_{j}} \cdot e^{-\epsilon_{1} t}
$$




$$
e^{-\Lambda_{1} t} \approx\left(1-\epsilon_{1} t_{j}\right) e^{-\lambda_{1} t} J,
$$

and in each datum equation,

$$
x_{1} e^{-\Lambda_{1} t_{j}}=x_{1} e^{-\lambda_{1} t_{j}}-\left(x_{1} \epsilon_{1}\right) t_{j} e^{-\lambda_{1} t_{j}} .
$$

We therefore replace each unknown $x_{1}$ with a pair of unknowns, $x_{1}$ and $\epsilon_{1} x_{1}$, and solve as for a system with twice the number of components. The $\epsilon_{1}$ are evaluated by division of the $\epsilon_{1} x_{1}$ by the $x_{1}$, the original values of the $\lambda_{1}$ are corrected by adding the $\epsilon_{1}$, and the process repeated. If the initial values of the $\lambda_{1}$ are sufficiently close to the true values so that equation VI 11 is a good approximation, the iterations will usually converge and the calculated values of the $\epsilon_{1}$ will approach zero

This technique is laborious at best, and beset with difficulties, if the original values of the $\lambda$ are not sufficiently good, the iteration will diverge instead of converging It is also possible to "overshoot" the true value of the $\lambda$ and encounter a situation where the successive values of a given $\epsilon_{2}$ oscillate about zero without decreasing.

Another approach is to consider the value of $x^{2}$ and its dependence on the $\lambda$, as derived in Chapter $V$, equation V.23. If $\chi^{2}$ is greater than its expected value, one may make the approximation that the deviation of $\chi^{2}$ from its expected value is

$$
\Delta\left(x^{2}\right) \approx \sum_{1=1}^{n} \frac{\partial\left(x^{2}\right)}{\partial \lambda_{1}} \cdot \Delta \lambda_{1}
$$

and select a set of $\Delta \lambda_{1}$ which will reduce $x^{2}$ to a minimum value. Any systematic scheme for selecting such a set may be used

A simple scheme is to as sume that the deviation of $x^{2}$ from its expected value is due to equal contributions from each $\lambda$, so that we select

$$
\Delta \lambda_{1}=\frac{\left(x^{2}\right)}{n\left[\frac{\partial\left(x^{2}\right)}{\partial \lambda_{1}}\right]}
$$

The coefficients of the $\left(\epsilon_{1} x_{1}\right)$ unknowns in the least-squares solution method are of course $t_{j} e^{-\lambda_{1} t_{j}}$ rather than simple exponential expressions 
Another simple scheme is to as sume that each $\lambda$ is in error by a fixed percentage, so that

$$
\Delta \lambda_{i}=\frac{\lambda_{i} \Delta\left(x^{2}\right)}{\sum_{i=1}^{n} \lambda_{i} \frac{\partial\left(x^{2}\right)}{\partial \lambda_{i}}} .
$$

Still another scheme is to weight the change in each $\lambda_{i}$ by the magnitude of the sensitivity of $x^{2}$ to that $\lambda_{i}$, $i_{\text {.ee. }}$ to change those $\lambda_{i}$ to which $\chi^{2}$ is most sensitive; this gives

$$
\Delta \lambda_{i}=\frac{\frac{\partial\left(\chi^{2}\right)}{\partial \lambda_{i}} \cdot \Delta\left(x^{2}\right)}{\sum_{i=1}^{n} \frac{\partial\left(x^{2}\right)}{\partial \lambda_{i}}} .
$$

Of these schemes, VI. 16 is probably better than VI.1 5 which is better than VI.14.

If any of the $\lambda$ 's of the system are known precisely, the se should not be varied in the iteration scheme. This situation may occur when it is desired to identify an impurity which is observed in a sample containing known components.

\section{Unknown Multicomponent Systems}

If nothing more is known about the sample than the raw data, consisting of a set of counting rates (and their variances) measured at various known times, the problem of resolution is in the general case extremely difficult. Two general methods of apprcach have been proposed.

The first method, which has occasionally been used, 16 is a "brute force" method in which one assumes in turn $n, n+1, n+2, \ldots$ components, evaluates their half-lives by an iterative procedure based on initially assumed values as outlined in the previous section, and then tests, each time, the statistical improvement in the fit to the data resulting from the addition of the latest component. When the improvement upon adding an additional component is no longer significant, one concludes that the latest added component is spurious and that the system was completely and correctly resolved in the

${ }^{6}$ Keepin, Wimett, and Ziegler, J. Nucl. Energy 6, 1 (1957). 
previous attempt. It goes without saying that such a method is extremely laborious, it is also somewhat liable to mistake two components with similar half-lives for a single component. The mathematical details of the method are discussed by Householder. 17

A more elaborate method has been described by Gardner et al. ${ }^{18}$ Their method is based on integral transforms. In brief, they attempt to determine a function $g(\lambda)$ which is a sum of delta functions, each multiplied by an appropriate amplitude, then if the activity of the sample is represented by $f(t)$,

$$
f(t)=\int_{0}^{\infty} g(\lambda) e^{-\lambda t} d \lambda .
$$

By a suitable transformation of variables, this equation 15 converted to the complex plane, $f(t)$ then assumes the form of a Fourier transform of $g(\lambda)$ By appropriate integrations, the inverse transformation can be performed and a plot of $g(\lambda)$ vs $\lambda$ is produced. (See Fig. VI.2.)

This method has the great advantage that the number of components appears as one result of the analysis. The actual plot is expected to resemble a spectrum, with a peak at each value of $\lambda$ corresponding to a component. The area of the peak is related to the abundance of the component. In actual practice, the situation is more complicated, one usually does not possess data which can be integrated to infinite time, and integration of numerical data is subject to errors. Both these effects contribute to the appearance of numerous small peaks, or "error ripples," which greatly confuse the spectrum. Many problems remain to be solved in the actual application of the method.

\section{DEAD TIME AND COINCIDENCE CONSIDERATIONS}

\section{Nature of the Problems}

Certain statistical problems arise from the physical limitations of the counting equipment itself. In general a single radiation event is detected by some physical system which is in a metastable state - a phototube with a potential across the electrodes, for example, or a wire at high potential in a gas. The event "triggers" the transition of this metastable state to a state

17 A. S. Householder, ORNL-455 (Unclassified).

18 D. G Gardner, J. C Gardner, G. Laush, and W W Meinke, J. Chem. Phys. 31, 978 (1959). 


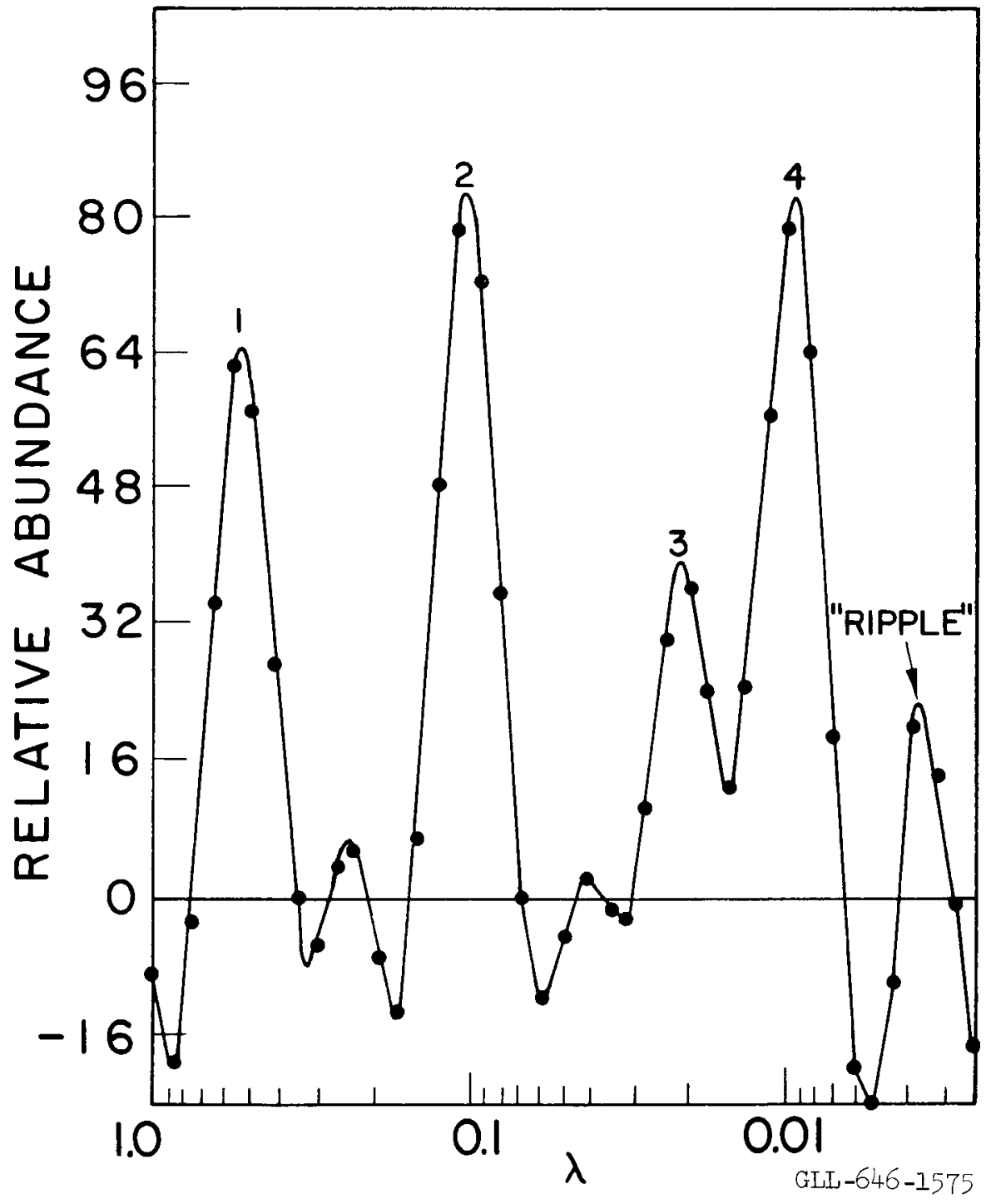

Fig VI 2. Result of the method of Gardner et al. applied to a set of synthetic data having four components. The four prominent peaks (numbered 1-4) represent components; minor error ripples about the zero-line are inherent in the method. The large ripple at the right-hand end represents fallure to obtain data out to infinite time. (Reproduced from Gardner et al., J Chem. Phys. 31, 978 (1959), Fig. 6, p. 983.) 
of lower potential energy, and the energy liberated in the transition is recorded. The system is then returned to its metastable state to await the next event. This process takes time; while the system is reacting to an event it is not capable of reacting to a second event. Two events occurring within a certain time, then, will be recorded as one event; if the system responds in a manner proportional to the energy of the arriving radiations, two events occurring within the critical time will record as a single event having the total energy of the two events. For counting circuits, this critical time is usually spoken of as the "dead time"; for coincidence circultry, as the "resolving time."

In this chapter we shall examine the effect of the dead time on the observed counting rate; the effect of scaling circuits on interval between pulses into a mechanical register; the measuring of true coincidences vs forturtous coincidences; determination of counting efficiency by coincidence methods; and the effect of coincident events on energy-discriminated counting circuits.

2. Effect of Dead Time on Observed Counting Rate

Rainwater and $\mathrm{Wu}^{8}$ have discussed two limiting situations. In one, $a$ pulse in the counter causes it to be insensitive for $\exists$ time $\tau$, a second event arriving during this time does not record and does not extend the dead time. In the second case, a second event arriving during the dead time does extend the dead time to a time $\tau$ after the arrival of the seccnd (undetected, event.

Let us consider the first situation. Let the "true' counting rate be $\mathrm{N}$ per unit time and the measured counting rate be $\mathrm{n}$ per unit time. Then since each recorded count implies a dead time of $\tau$, which is $n=t$ affected by the unrecorded events, the total time lost per unit time $1 \mathrm{~s} n \tau$. The number of events occurring during this lost time is NnT, so that

$$
N-n=N n \tau \text {. }
$$

Consequently, for known dead tıme.

$$
\mathrm{N}=\frac{\mathrm{n}}{\mathrm{l}-\mathrm{n} \tau}
$$

is fitted to

$$
\sum_{y} x e^{-\lambda} y^{t} i
$$


Since

$$
\mathrm{n}=\frac{\mathrm{N}}{\mathrm{l}+\mathrm{N} \tau}
$$

It can be seen that the maximum counting rate in this type of instrument, as $\mathrm{N}$ increases without limit, is $1 / \tau$.

In the second case, where the dead time is extended by $\tau$ for each event, whether recorded or not, the analysis is slightly more elaborate. One can see that a second count will be recorded only if it follows the first count by a time interval greater than $\tau$. We must consider the probability that the interval between events, arriving at a rate $N$ per unit time, is at least as great as $\tau$.

The expected number of events during an interval $\tau$ is $\mathrm{N} \tau$ If Poisson statistics applies, the probability that the actual number is zero, that is, that a second event does not arrive within a time $\tau$, is given by

$$
\mathrm{P}_{0}(\mathrm{n})=\frac{(\mathrm{N} \tau)^{0} \mathrm{e}^{-\mathrm{N} \tau}}{0 !}=\mathrm{e}^{-\mathrm{N} \tau}
$$

Consequently, the fraction of the time that the counter is "live" is $\mathrm{e}^{-\mathrm{N} \tau}$; the observed counting rate will be

$$
\mathrm{n}=\mathrm{N} \mathrm{e}^{-\mathrm{N} \tau} \text {, }
$$

which for small values of $\mathrm{N} \tau$ is approximately equivalent to equation Vir. 3 For large values of $N_{T}, n$ in equation VII.5 goes to zero - the counter is "blocked" or saturated. The maximum counting rate can be seen to occur at $\mathrm{N} \tau=1$, where $\mathrm{n}$ is equal to $1 / \tau$ e.

In actual practice, the observed behavior of a system is usually intermediate between the two extremes. Unless a great deal of effort is expended on checking the behavior of the system at high count rates, it is unwise to operate in a region where $\mathrm{N} \tau$ is appreciable compared to 1, even if the "dead time" is reputedly known, since when equation VII 5 diverges appreciably from equation VII.3, it is not certain what the actual correction should be unless the actual behavior of the system has been carefully examined. 
There are several general methods of measuring counter dead time The simplest method (in principle) involves the preparation of a series of long-lived standard samples whose counting rates relative to each other are known a priori, the observed ratios between counting rates are then used with equation VII 2 to give the dead time. Since

$$
\begin{aligned}
& \mathrm{N}_{1}=\frac{\mathrm{n}_{1}}{1-\mathrm{n}_{1} \tau}, \\
& \mathrm{N}_{2}=\frac{\mathrm{n}_{2}}{1-\mathrm{n}_{2} r},
\end{aligned}
$$

then combining these two equations and solving for $\tau$,

$$
\tau=\frac{\mathrm{n}_{1} / \mathrm{n}_{2}-\mathrm{N}_{1} / \mathrm{N}_{2}}{\mathrm{n}_{1}\left(1-\mathrm{N}_{1} / \mathrm{N}_{2}\right)}=\frac{\mathrm{n}_{2} / \mathrm{n}_{1}-\mathrm{N}_{2} / \mathrm{N}_{1}}{\mathrm{n}_{2}\left(1-\mathrm{N}_{2} / \mathrm{N}_{1}\right)} .
$$

A set of suitable samples may be prepared by pipetting various carefully measured aliquots of a suitable tracer onto identical backings, making sure that the size and location (on the backing) of each spot is identical with all the others, and that the amount of solid material present is constant. If the samples are not identical in all respects, errors in positioning within the counter and nonidentity of sample scattering and absorption on the various samples will perturb the result. It should be noted that, since evaluation of dead time by a relationship 1 ike equation VII 2 is only valid for $N \tau<$ ], we are attempting to measure a small deviation from ideal behavior, such a measurement is equivalent to measuring a small difference between large numbers, which (refer to Theorem IV, Chapter III) is subject to large variance if the numbers are statistically independent, as they are in this case.

Another common technique based on the same approach involves the preparation of a divided sample, so mounted that either portion may be inserted in the counter, or both may be inserted together without either portion interfering with the other. If $n_{1}$ is the observed counting rate from one portion, $\mathrm{n}_{2}$ is the observed counting rate for the other, and $\mathrm{n}_{12}$ is the observed counting rate for both samples together, we have for the true counting rates 


$$
\begin{aligned}
& N_{1}=\frac{n_{1}}{1-n_{1}^{\top}}, \\
& N_{2}=\frac{n_{2}}{1-\frac{n_{2}}{T}}, \\
& N_{12}=\frac{n_{12}}{1-n_{12}{ }^{\top}}=N_{1}+N_{2} .
\end{aligned}
$$

Substituting VII.8a and b into VI..8c, we get

$$
\frac{\mathrm{n}_{12}}{1-\mathrm{n}_{12} 2^{\tau}}=\frac{\mathrm{n}_{1}}{1-\mathrm{n}_{1} \tau}+\frac{\mathrm{n}_{2}}{1-\mathrm{n}_{2}{ }^{\tau}}
$$

which upon simplifying and solving gives

$$
\tau=\frac{n_{1} n_{2} \pm \sqrt{n_{1} n_{2}\left(n_{12}-n_{1}\right)\left(n_{12}-n_{2}\right)}}{n_{1} n_{2} n_{12}} .
$$

It can be shown that choice of the upper sign will result in a negative answer for $\mathrm{N}_{\text {] }}$ or $\mathrm{N}_{2}$. consequently we have

$$
\tau=\frac{n_{1} n_{2}-\sqrt{n_{1} n_{2}\left(n_{12}-n_{1}\right)\left(n_{12}-n_{2}\right)}}{n_{i} n_{2} n_{12}} .
$$

This technique appears simple in theory, in practice, extreme care must be used to ensure that each portion of the split sample is counted separately in exactly the same position it occupies when the portions are counted together. The term under the radical sign does not differ greatly from $n_{1}{ }_{2}$; we are again faced with a small difference between large numbers. Repeated measurements should be made with different sets of split samples; counts should be taken with high precision, recording as many events as possible during each of the three measurements.

A second method for measuring dead time involves the preparation of a strong sample of a short-lived single species whose half-life is very well known. ( $\mathrm{C}^{\mathrm{ll}}$, with a half-life of 20.5 minutes, is a convenient species for the purpose.) If the initial activity of the sample is high enough so that dead time losses are appreciable, then the activity will not decay exponentially with time; if $\mathrm{n}$ is the observed counting rate, and the true counting rate decays as a single-component exponential, then 


$$
n_{1}=\frac{N_{0} e^{-\lambda t} 1}{1+N_{0} \tau e^{-\lambda t}}
$$

and if enough points are available the parameters $N$ and $\tau$ can be evaluated either by a least-squares method or graphically.

A simple graphical approach to give an approximate answer may be derived by reciprocating equation VII 12 to give

$$
\frac{1}{n_{1}}=\frac{1}{N_{0}} e^{+\lambda t_{1}}+\tau
$$

If $l / n_{1}$ is plotted against exp $\left(+\lambda t_{1}\right)$ a straight line should be obtained with a slope of $1 / \mathrm{N}_{0}$ and an intercept of $\tau$. The same form of the equation can readily be used for a least-squares solution, providing that the variances of the $\mathrm{l} / \mathrm{n}_{1}$ can be derived This is done as follows: rewriting VII.] 3 as

$$
\frac{1}{n_{1}}=\frac{1}{N_{1}}+\tau,
$$

then

$$
\begin{aligned}
& \operatorname{var}\left(\frac{1}{n_{1}}\right)=\operatorname{var}\left(\frac{1}{N_{1}}\right)=\frac{1}{N_{1}^{2}} \operatorname{var}\left(N_{1}\right)=\frac{N_{1}}{\Delta t_{1} N_{1}^{2}}, \\
& \operatorname{var}\left(\frac{1}{n_{1}}\right)=\frac{1}{N_{1} \cdot \Delta t_{1}},
\end{aligned}
$$

where $\Delta t_{1}$ is the counting interval (see Chapter $V$, equation V.7) Unfortunately, $\mathrm{N}_{1}$ involves the parameter $T$, we are again involved in an iterative calculation, where a first approximation for $\tau$ is obtained in some reasonable fashion, the weighting factors for the least-squares solution are computed, using this initial value, a new value for $\tau$ is obtained by least-squares analysis, if this value is appreciably different from the initial value, the new value of $T$ is used to recompute improved weighting factors and the procedure is repeated until the value of $\tau$ converges to its best value. Since $1 / \mathrm{n}_{1}$ is expected to be considerably greater than $\tau$ if the simple concept of dead time is to have meaning, the convergence should be quite rapid.

One may also estimate dead time by electronic methods, if the output of the counter is used to trigger the timed sweep of an oscilloscope, and the 
counter pulse is displayed vertically on the scope, then if a highly active source is put into the counter the scope screen will display a pattern like that in Fig VII I (in the 1deal case) In Fig. VII.l the first pulse is displayed at the left end of the scope trace, pulses which follow during the sweep of the scope will appear at random at any point of the sweep after termination of the dead time If a large number of sweep patterns are superposed, as for example by taking a time-exposure photograph of a number of sweeps, the dead time may be measured by the width of the gap between the first pulse and the earliest second pulse $T$ is sometimes a function of pulse height.

Electronic circuits are becoming avalable which measure counting time intervals in terms of "live time." When such equipment is available, or where the dead time is so short or the counting rate so low that dead time losses are negligible, much trouble may be avoided.

\section{Scaling Circuits - Avoiding Losses in Slow Components}

If the counting instrument embodies a mechanical register or other slow-acting component (very few mechanical registers will accept pulses separated by only 0 ] second) serious trouble may arise. Even when the average interval between pulses is long enough to permit response of the register, the statistical distribution in time of the pulses implies that a certain fraction of the pulses will occur too close together to be resolved. It is customary to use an electronic scaling circuit between the counter and the register, so that for every $m$ events in the counter only one pulse is fed to the register. This obviously reduces the register counting rate, and increases the average interval between pulses, by a factor of $m$, we shall show, following the treatment of Rainwater and $\mathrm{Wu},{ }^{8}$ that there $1 \mathrm{~s}$ an additional beneficial effect gained from the regularizing action of such a scaling circuit on the output pulse rate. In other words, the distribution of intervals between output pulses from a scale-of-m circuit is narrower than that from a counter counting at the same rate, $1 . e$, with the same average spacing between pulses. This implies that a given register might be satisfactory, used with a counting rate of $\mathrm{mN}$ and a scale-of-m circuit, even though the losses due to slow register response would be unacceptable if the same register were used with a counting rate of $\mathrm{N}$ and no scaling circuit

We wish to consider the probability $\mathrm{q}_{\mathrm{m}}(\mathrm{x}) \mathrm{dx}$ that the actual interval between two output pulses from the scaling circuit lies between $\mathrm{x}$ and $\mathrm{x}+\mathrm{dx}$, 


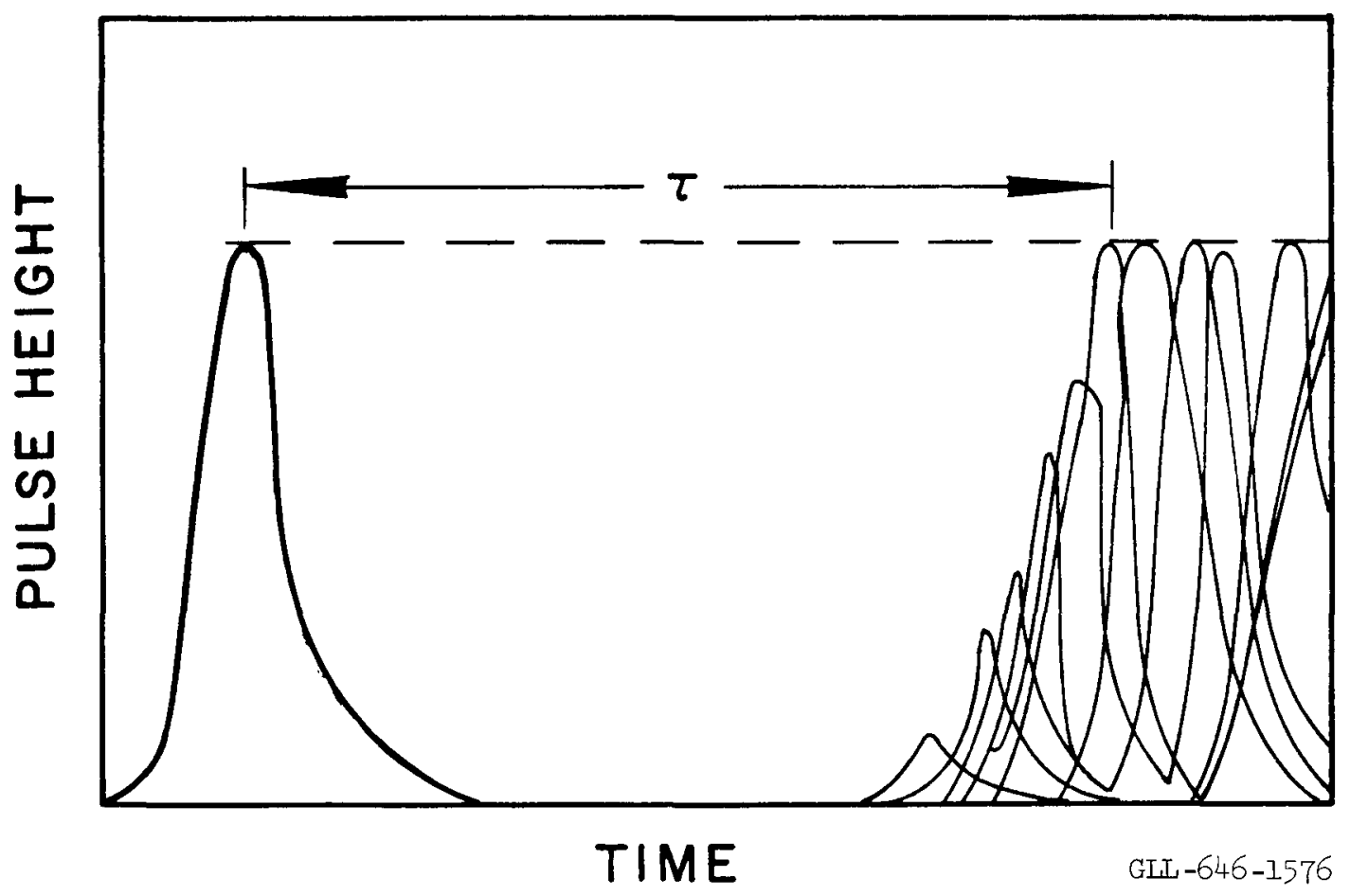

Fig. VII. 1. Schematic representation of the measurement of counter dead time $\tau$ using an oscilloscope. Pulses from the counter are used to trigger the horizontal sweep of the oscilloscope. Events which occur within the sweep time appear as peaks in the right-hand portion of the trace. Repeated traces are recorded, e.g. by a long-exposure photograph; counter dead time results in a blank region in which no record pulses appear. 
where $\mathrm{x}$ is time measured in units of the average spacing between output pulses, i.e., if the output pulse rate is $\mathrm{N}$, and the input pulse rate is $\mathrm{mN}$, then for a time $t, x$ is equal to Nt. We shall start by considering the probability $Q_{m}(x)$ that an output pulse will occur within a time $t$ of the preceding output pulse.

During the time interval $t$ the scaling circuit will receive some number $\mathrm{n}$ of input counts. Let us assume that Poisson statistics applies; then the probability of $\mathrm{n}$ input counts is given by

$$
P_{n}=\frac{\overline{E(n)})^{n} e^{-E(n)}}{n !}
$$

and the expected number of counts is

$$
\mathrm{E}(\mathrm{n})=\mathrm{mNt}=\mathrm{mx} \text {. }
$$

An output pulse will have been obtained if $n \geq m$. Consequently the probability of obtaining an output pulse is the sum of all $P_{n}$ for $n \geq m$; or

$$
Q_{m}(x)=\sum_{n=m}^{\infty} P_{n}(m x)=1-\sum_{n=0}^{m-1} P_{n}(m x)
$$

The probability that an output pulse will occur during the interval between $x$ and $x+d x$ is the change in $Q_{m}(x)$ when $x$ increases from $x$ to $x+d x$; in other words

$$
q_{m}(x)=\frac{d}{d x}\left[Q_{m}(x)\right]
$$

Substituting equations VII. 17 and VII. 18 in equation VII.19, we get

$$
\begin{aligned}
& q_{m}(x)=\frac{d}{d x}\left[1-\sum_{m=0}^{m-1} \frac{(m x)^{n} e^{-m x}}{n !}\right], \\
& q_{m}(x)=m \cdot \frac{(m x)^{m-1} e^{-m x}}{(m-1) !}
\end{aligned}
$$


If this is written

$$
\mathrm{q}_{\mathrm{m}}(\mathrm{x}) \mathrm{dx}=\frac{(\mathrm{mx})^{\mathrm{m}-1} \mathrm{e}^{-\mathrm{mx}}}{(\mathrm{m}-1) !} \mathrm{d}(\mathrm{mx})
$$

it is apparent that it corresponds to the form of a Poisson distribution, with an expected value of $m x$ equal to $m-1+1=m$, so that $E(x)=1$ as $m i g h t$ be expected; the point of principal interest is that the width of this distribution, as measured by its variance, is also $\mathrm{m}$; so that

$$
\begin{aligned}
\operatorname{va}(m x) & =m^{2} \operatorname{var}(x)=m, \\
\operatorname{var}(x) & =\frac{1}{m},
\end{aligned}
$$

and the width of the distribution in $\mathrm{x}$ varies inversely as $\mathrm{m}$. Figure VII.2 shows the shapes of the distributions for several values of $\mathrm{m}$, including $\mathrm{m}=1$ (no scaling). The curves are normalized so that the area under the curve for $\mathrm{x} \leq \mathrm{x}_{1}$ is the probability that the interval between consecutive output pulses is $\mathrm{x} \leq \mathrm{x}_{\mathrm{l}}$. The very small areas under the curves for small values of $\mathrm{x}$ when a scaling circuit is used should be compared to the large area under the curve for $m=1$, i.e., no scaling; this clearly demonstrates the advantage to be gained by the use of even small scaling factors.

\section{Measurement of Coincident Events}

It is sometimes desired to measure events which are "coincident" in the sense that they occur, one in each of two counters, within a certain time of each other. It is of course meaningless to require that the two events occur at precisely the same instant; the uncertainty principle alone implies a certain imprecision in the time of occurrence of events of finite energy. In addition, any real physical instrument for measuring coincident events has a finite resolving time $\tau$; events occurring within a time $\tau$ of each other will be recorded as coincident.

Events measured as coincident may be divided into two classes: "truly coincident" events which are related in some physical way, such as two gamma photons emitted in cascade without noticeable delay in the intermediate state; and "chance coincident" events, which arrive together at the detection system within the time $\tau \mathrm{through}$ pure chance. Fortunately, it is possible to calculate the rate at which chance coincident events will occur; the gross rate of 


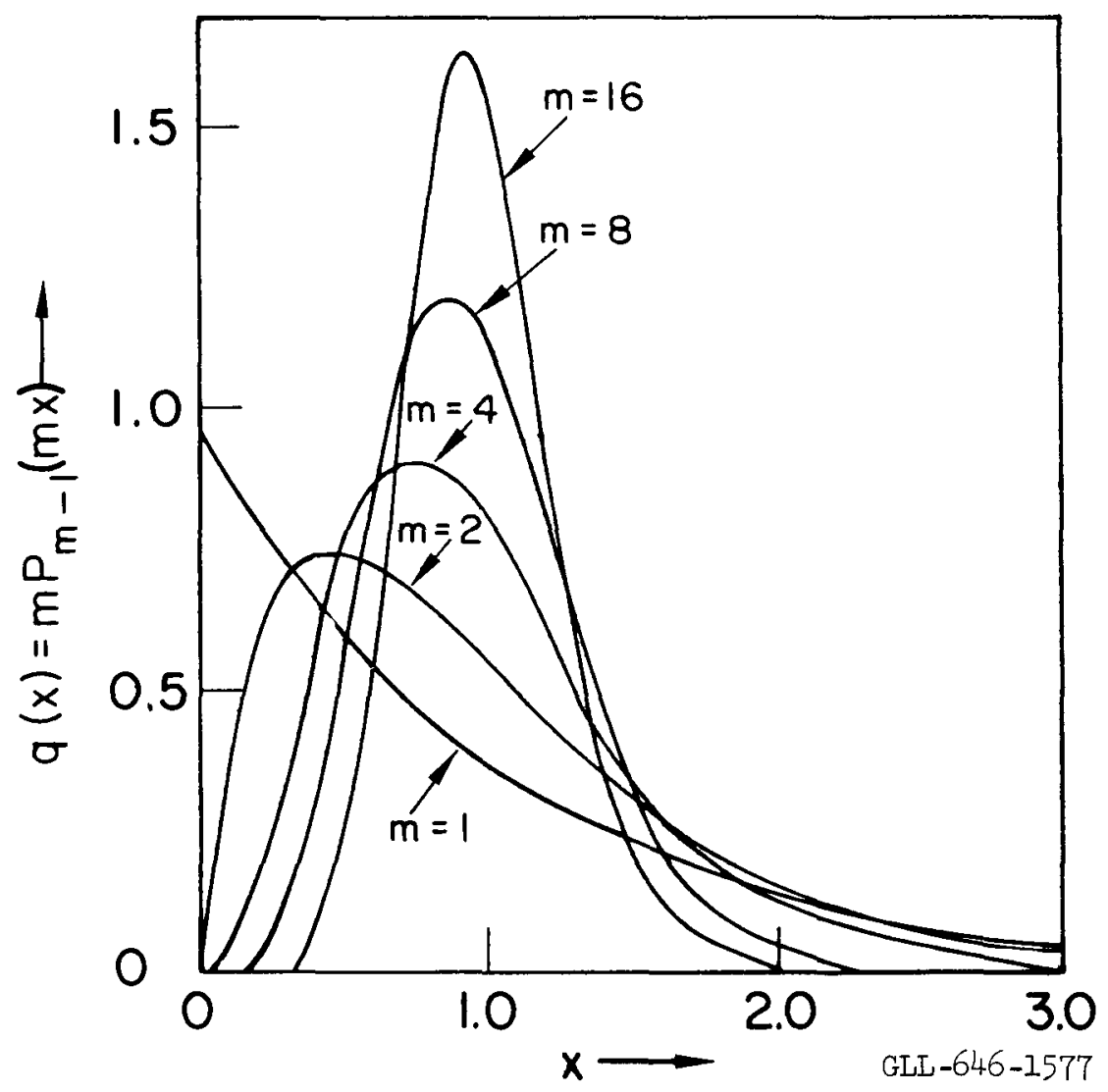

Fig. VII.2. Regularizing action of scaling circuits. $q(x)$ dx represents the probability that the time interval between two pulses to the register will lie between $x$ and $x+d x$, where $x$ is a fraction of the mean interval. The probability that the interval will be less than the dead time, a, of the register is represented by the area under the curve from $x=0$ to $x=a ; m$ is the scaling factor in the scaling circuit. It can readily be seen that even rather small scaling factors are very helpful in reducing losses. (Reproduced from Rainwater and $\mathrm{Wu}$, Nucleonics, October 1947, p. 60; Fig. 3, p. 69.) 
coincident events can then be corrected to give the true coincidence rate. The calculation is made as follows. Let us assume that, as is almost always true, the true coincidence counting rate is very small compared to the counting rate in either counter. If the counting rate in one counter is $\mathrm{n}_{1}$, and the counting rate in the second counter is $n_{2}$, then the probability of a count in one counter during the interval $\tau$ is proportional to both $\mathrm{n}_{2}$ and $\tau$, it 1 , in fact, $\mathrm{n}_{2} \tau$. The usual circuit for coincidence measurements is so arranged that a coincidence will be recorded if a count in counter 2 follows a count in counter 1 within an interval $\tau$, but not the reverse, in such a case, the chance coincidence rate will be $n_{1} n_{2}$. If $n_{1}$ and $n_{2}$ are recorded and $\tau$ is known, the chance coinc1dence rate can readily be calculated.

In planning an experiment it is desirable to make an estimate of the ratio of true to chance coincidences. For this we need an estimate of the counting efficiency of each counter. If the true disintegration rate $1 \mathrm{~N}$, and the efficiencies in each counter are $\eta_{1}$ and $\eta_{2}$ where efficiency in this case is taken to include branching ratios, decay schemes, and selective counting response, then the true coincidence rate can be seen to be $\eta_{1} \eta_{2} \mathrm{~N}$ and the chance coincidence rate can be seen to be $\eta_{1} \eta_{2} \mathrm{~N}^{2} \tau$. The ratio of "true" to "chance" coincidences will then be $1 / \mathrm{N} \tau$. It is therefore desirable to operate with as short a resolving time as possible, which seems obvious a priori, what is not so obvious is that it is also advantageous from the point of discriminating against false coincidences to operate with a sample containing as little activity as possible

If this is done, it then becomes highly desirable to increase the solid angle intercepted by the counters to the largest possible value so that the observed gross counting rate will be as high as possible, in order to obtain good statistical reliability in a reasonable experimental period. We are now in some danger of violating our simplifying assumption, if we consider a limiting case in which every count in the first counter gives rise to a true coincident count in the second counter, it is obvious that while $\tau \mathrm{n}_{1} \mathrm{n}_{2}$ is not zero the actual false coincidence rate is truly zero.

The evaluation of true versus false coincident counts is somewhat more complicated if we omit the simplifying assumption. Let us assume a "true coincident" counting rate of $\mathrm{n}_{12}$, then there will be $\mathrm{n}_{12}$ counts per unit time in both counters 1 and 2 which should not be included in the evaluation of the 
false rate If $g$ is the gross observed coincident count rate, and $f$ is the false coincident rate, then

$$
g=f+n_{12}
$$

and

$$
f=\left(n_{1}-n_{12}\right)\left(n_{2}-n_{12}\right) \tau
$$

so that, combining VII.26 and VII 27 ,

$$
n_{12}=\frac{\tau\left(n_{1}+n_{2}\right)-1+\sqrt{\left[1-\tau\left(n_{1}+n_{2}\right)\right]^{2}+4 \tau\left(g-\tau n_{1} n_{2}\right)}}{2_{\tau}} .
$$

This ran be transformed to

$$
n_{12}=\left[\frac{1-\tau\left(n_{1}+n_{2}\right)}{2 \tau}\right]\left[-1+\sqrt{1+\frac{4 \tau\left(g-\tau n_{1} n_{2}\right)}{\left[1-\tau\left(n_{1}+n_{2}\right)\right]^{2}}}\right]
$$

which can be approximated by

$$
\mathrm{n}_{12} \rightarrow \frac{\mathrm{g}-\tau \mathrm{n}_{1} \mathrm{n}_{2}}{1-\tau\left(\mathrm{n}_{1}+\mathrm{n}_{2}\right)} \text { as }\left[\frac{4 \tau\left(\mathrm{g}-\tau \mathrm{n}_{1} \mathrm{n}_{2}\right.}{\left[1-\tau\left(\mathrm{n}_{1}+\mathrm{n}_{2}\right)\right]^{2}}\right] \text { becomes }<1 \text {. }
$$

Since for any reasonable system $\tau\left(n_{1}+n_{2}\right) \ll 1$, then the simplifying assumption does not give rise to serious error unless $4 \tau n_{12}$ is appreciable compared to 1 .

\section{Variance of Coincident Event Rate}

If one considers a system such as the above, but containing a sample of such a nature that there are no true coincidences, then it becomes apparent that the gross coincidence count rate observed must be the actual false rate, that the expected value of the difference between the observed gross rate and the calculated false rate is zero, and that the variance of the difference between the gross and the calculated false rates is just the variance of the false coincidence rate. This variance can be computed from theory.

Since we know that the expected value of the false rate, $f$, is given (to a good approximation) by

$$
\mathrm{f}=\operatorname{\tau n}_{1} \mathrm{n}_{2}
$$

then 


$$
\operatorname{var}(\mathrm{f})=\tau^{2} \operatorname{var}\left(\mathrm{n}_{1} \mathrm{n}_{2}\right)=\tau^{2} \mathrm{n}_{1} \mathrm{n}_{2}\left(\frac{\mathrm{n}_{1}+\mathrm{n}_{2}}{\mathrm{t}}\right)
$$

where $t$ is the duration of the count (see Chapter V, equation V.7). Since for true coincidences Poisson statistics should apply,

$$
\operatorname{var}\left(\mathrm{n}_{12}\right)=\frac{\mathrm{n}_{12}}{\mathrm{t}} \text { if } \mathrm{f}=0 \text {, }
$$

then from the preceding discussion

$$
\operatorname{var}\left(\mathrm{n}_{12}\right)=\frac{g_{-1}}{t}+\tau^{2} n_{1} n_{2}\left(\frac{n_{1}+n_{2}}{t}\right) .
$$

This variance is then used for least-squares weighting.

\section{The Effect of Coincident Events on Discriminated Counts}

- Several varieties of counters may be made to give an output pulse with amplitude nearly proportioned to the energy of the event giving rise to the pulse. Such counters, in conjunction with suitable discriminating or analyzing circuits, may be used to give particle energy spectra, or to count radiations of a particular energy in the presence of a variety of others. In this situation coincident events may occur in which two particles enter the counter within the resolving time of the system, the amplitude of the resulting pulse is then proportional to the sum of the energies of the two particles.

When one is examining a sample which gives rise to a pair of truly coincident events - for example, two gamma photons in cascade - then if the probability of one event recording in the counter is $\mathrm{p}_{1}$, and the probability of the other recording in the counter is $p_{2}$, the probability of both events recording in the counter is $\mathrm{p}_{1} \mathrm{p}_{2}$. Consequently, the ratio of coincident events, giving rise to a "sum" peak with energy $E_{1}+E_{2}$, to single events of one type (e.g, type 1) will be

$$
\text { Sums/single }=\frac{p_{1} p_{2}}{p_{1}-p_{1} p_{2}}-\frac{p_{2}}{1-p_{2}} \approx p_{2} \text {. }
$$

$p_{1}$ and $p_{2}$ are simply the counting efficiencies of the system for the types of radiation in question, consequently, for a given decay rate, $\mathrm{p}_{2}$ is proportional to the singles counting rate of the second type of event. Typical counting efficiencies are $\geq 10^{-3}$ in most cases and may even approach unity in special arrangements 
Let us now consider a sample which does not give rise to truly coincident events, in a system with resolving time 7 . The relationship between count rates for single events and count rate for false coincidences is the same as that derived in the previous section; the ratio between "false sums" and singles of type I will be

$$
\text { Sums } / \text { singles }=\frac{\mathrm{n}_{1} \mathrm{n}_{2}{ }^{\top}}{\mathrm{n}_{1}}=\mathrm{n}_{2}{ }^{\top}=\mathrm{p}_{2} \mathrm{~N}
$$

(where $\mathrm{N}$ is the disintegration rate), which is again proportional to the count rate of the other type of event. In this case the two types of events may be photons of different energy originating from different atoms, giving rise to a "sum peak" of energy $E_{1}+E_{2}$, or photons of the same energy, giving rise to a "sum peak" of energy $2 \mathrm{E}_{1}$, in which case $\mathrm{N}_{2}$ and $\mathrm{N}_{1}$ are equal. It will be seen that there are differences between true and false sum peaks. The size of the proportionality factor is larger in general for true coincidences; but much more important is the fact that $\mathrm{p}_{2}$ depends only on solid angle, counter characteristics, and to a slight extent on sample mounting, so that the ratio of sums to singles in a particular counting arrangement depends only on counting position, i.e., solid angle, for true coincidences, but depends on absolute disintegration rate for false coincidences. The sums-to-singles ratio will therefore change with time as the sample decays and the absolute disintegration rate changes.

A point that should be made is that a nuclear species which has two gamma photons in coincidence may actually have a single gamma photon whose energy is the sum of the two coincident gammas; the decay scheme for such a species is drawn in Fig. VII.3. The gamma spectrum of such a species will contain a peak of energy $E_{1}+E_{2}$ which will arise from three sources: single events corresponding to the "crossover" transition from the upper level to the ground state; true coincident counts of the two gammas in cascade; and false coincidences resulting when one of the cascade gammas strikes the counter in fortuitous coincidence with the cascade gamma of complementary energy from another nucleus. In attempting to demonstrate the real existence of the "crossover" transition, and to measure its abundance, due allowance must be made for contributions to the gamma peak from the other sources.

It should also be noted that a multicomponent sample is sometimes followed for decay in a proportional counter with a discriminator set to block 


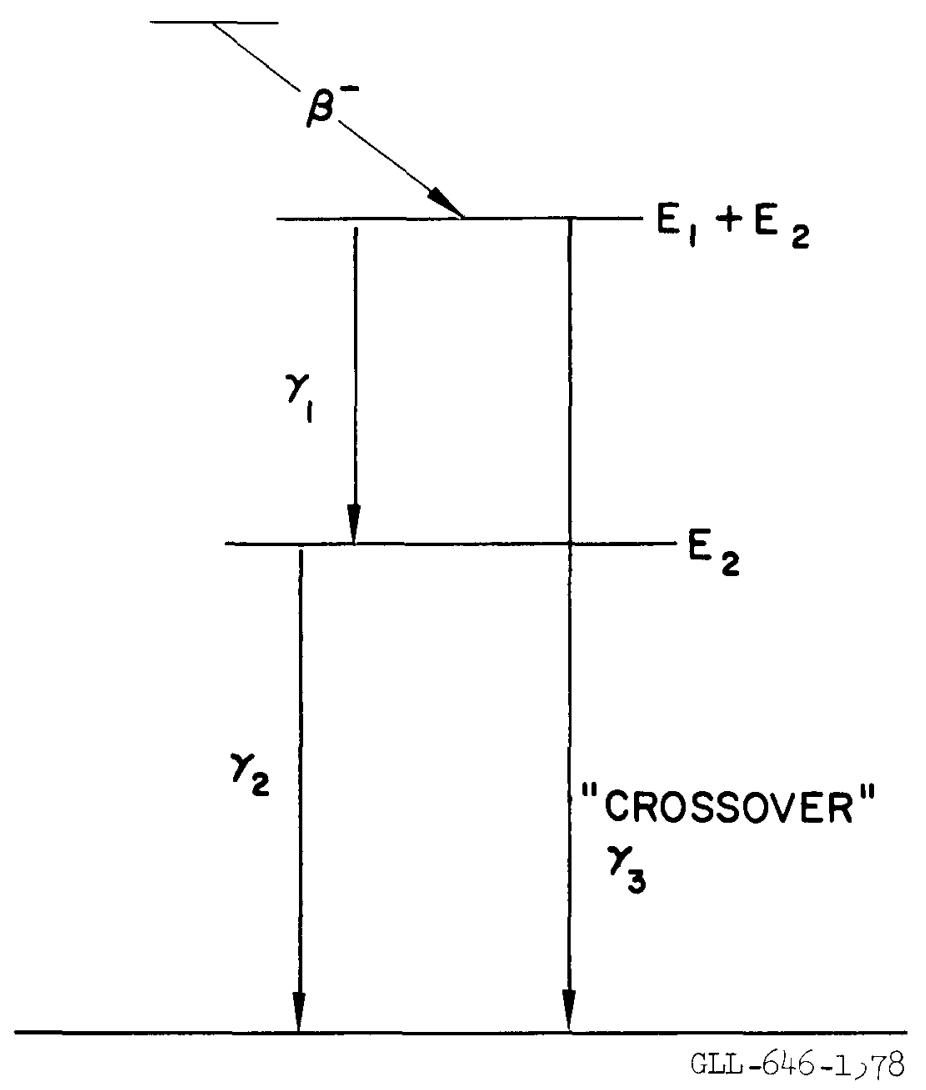

Fig. VII.3. Decay scheme illustrating a possible difficulty in interpreting "sum peaks" in gamma spectra. A peak will appear in the spectrum of such a nuclide at the gamma energy $E_{1}+E_{2}$. This arises from three sources a "true coincidence" when the photons $\gamma_{1}$ and $\gamma_{2}$ both strike the crystal, a "crossover" when the nuclide decays directly from the upper excited state to the ground state, and a "false coincidence" when a photon of energy $\mathrm{E}_{1}$ from one nucleus and a photon of energy $E_{2}$ from another both strike the crystal fortuitously within the resolving time of the instrument The intensity of the "sum peak" will vary with solid angle and with absolute source intensity in different ways for the three different phenomena. 
off a component which gives rise only to relatıvely low-energy radiations, while recording the higher energy radiations from another component If there are true coincidences in the undesired species, the "sum peak" may 1ie above the discriminator level and the unwanted component will appear in the decay curve. If there are no coincident events, but the unwanted component is sufficiently active to give an appreciable number of false coincidences, it will easily be seen that the false coincident rate will depend on the square of the count rate, the decay rate for the false sum peak will be given by

$$
R \propto\left(R_{0} e^{-\lambda t}\right)^{2}=R_{0}^{2} e^{-2 \lambda t}
$$

and a component will appear in the decay having precisely half the half-life of the supposedly eliminated species This effect can be quite startling if not anticipated.

\section{Measurement of Absolute Counting Efficiency by Coincidence Techniques}

If one has a radioactive species which gives rise to truly coincident events, measured in an experimental arrangement of two counters, it is possible to use the coincidence counting rate to measure the true disintegration rate of the sample. If the counting efficiencies in the two counters, including solid angle and branching ratio if necessary, are $\eta_{1}$ and $\eta_{2}$, then the counting rates are given by

$$
\begin{aligned}
\mathrm{n}_{1} & =\eta_{1} \mathrm{~N}, \\
\mathrm{n}_{2} & =\eta_{2} \mathrm{~N}_{2} \\
\mathrm{n}_{12} & =\eta_{1} \eta_{2} \mathrm{~N}+\tau \mathrm{n}_{1} \mathrm{n}_{2} \quad \text { (gross coincidence rate), }
\end{aligned}
$$

and consequently

$$
N=\frac{n_{1} n_{2}}{n_{12}-\tau n_{1} n_{2}} .
$$

It is of some interest to consider the precision of such a measurement. We have seen (equation VII 34) that 


$$
\operatorname{var}\left(\mathrm{n}_{12}-\tau \mathrm{n}_{1} \mathrm{n}_{2}\right)=\frac{\mathrm{n}_{12}-\tau \mathrm{n}_{1} \mathrm{n}_{2}}{\mathrm{t}}+\frac{\tau^{2} \mathrm{n}_{1} \mathrm{n}_{2}\left(\mathrm{n}_{1}+\mathrm{n}_{2}\right)}{\mathrm{t}}
$$

and consequently, using Theorems V and VI of Chapter III, we get, where $t$ is the duration of the measurement,

$$
\operatorname{var}(N)=\frac{1}{t}\left(\frac{n_{1} n_{2}}{n_{12}-\tau n_{1} n_{2}}\right)^{2}\left[\frac{1}{n_{1}}+\frac{1}{n_{2}}+\frac{1}{n_{12}-\tau n_{1} n_{2}}+\frac{\tau^{2} n_{1} n_{2}\left(n_{1}+n_{2}\right)}{\left(n_{12}-\tau n_{1} n_{2}\right)^{2}}\right] .
$$

(VII.41) 


\section{GLOSSARY OF SYMBOLS}

\begin{tabular}{|c|c|}
\hline $\begin{array}{l}|A| \\
A^{-1}\end{array}$ & $\begin{array}{l}\text { determinant of the matrix } A \\
\text { inverse of the matrix } A\end{array}$ \\
\hline$A^{\dagger}$ & transpose of the matrix A \\
\hline & element of the matxix A \\
\hline & coefficient in a system of linear equations \\
\hline$b, \mathrm{~B}$ & total number of background events \\
\hline $\mathrm{B}, \mathrm{C}, \mathrm{H}$ & matrices \\
\hline $\operatorname{cov}(x, y)$ & covariance of twc variates $x$ and $y$ \\
\hline $\mathrm{D}$ & diagonal matrix \\
\hline$E(x)$ & expected value of a variate $x$ \\
\hline$E(f(x))$ & expected value of a function of a variate $x$ \\
\hline $\mathrm{f}$ & false coincidence rate \\
\hline $\mathrm{F}_{\mathrm{e}}$ & extrapolation factor \\
\hline g & $\begin{array}{l}\text { growth factor, gross counting rate, events/unit time, right } \\
\text { member vector of the normal equations, gross number of events, } \\
\text { gross observed coincidence rate }\end{array}$ \\
\hline$g(\lambda)$ & function used to resolve unknown mixtures (see p. 107) \\
\hline G & $\begin{array}{l}\text { vector of right member of normal equations, gross number of } \\
\text { events }\end{array}$ \\
\hline 1 & vector with unit components \\
\hline$I$ & unit matrix \\
\hline $\mathrm{k}$ & number of replıcate samples or measurements \\
\hline $\mathrm{K}_{\mathrm{x}}$ & covariance matrix of a variate vector \\
\hline $\mathrm{m}$ & number of measured data points \\
\hline $\mathrm{n}$ & $\begin{array}{l}\text { number of atoms in sample, number of components in system, } \\
\text { number of observed events, counting rate modified by dead time }\end{array}$ \\
\hline $\mathrm{N}$ & number of atoms in sample, true counting rate \\
\hline$n \phi$ & integrated flux over the sample \\
\hline $\mathrm{O}$ & zero matrix \\
\hline $\mathrm{p}$ & $\begin{array}{l}\text { observed-calculated number, probabılity that an atom will decay } \\
\text { and give rise to a count }\end{array}$ \\
\hline$P_{x}, P_{x}(u)$ & distribution function of $x$ (along cocrdinate $u$ ) \\
\hline $\mathrm{q}_{\mathrm{m}}(\mathrm{x})$ & probability distribution function for intervals between events \\
\hline$Q_{m}(x)$ & probability distribution function for output pulses (integral) \\
\hline & special polynomial defined in eq. IV. 25, p. 69 \\
\hline
\end{tabular}




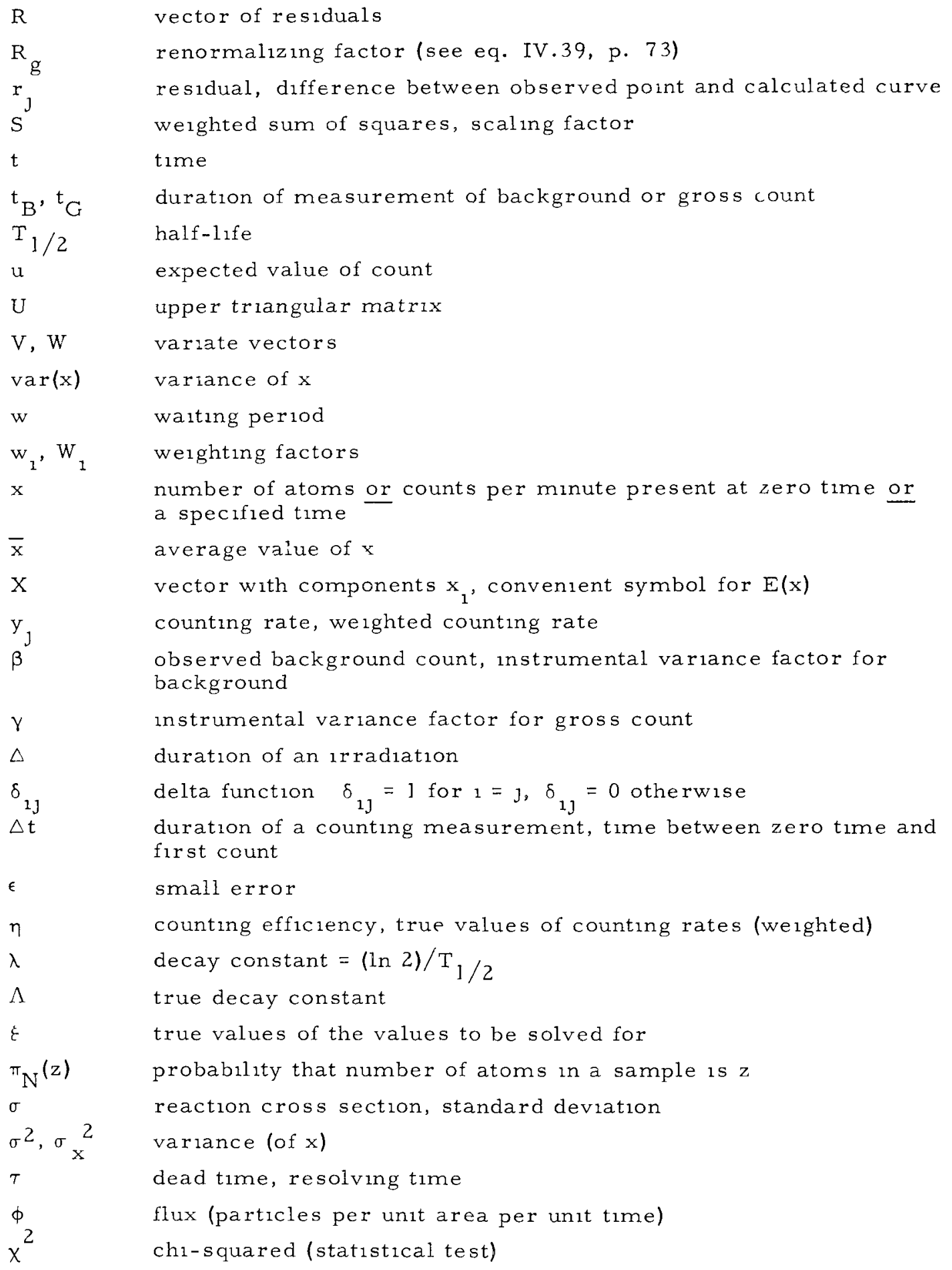


IX. INDEX

Page No.

Absolute counting

measurement of --- efficiency by coincidence techniques

precision of --- by coincidence

Absorbing

radiation scattering and ---

Activation analysis

Activities

use of daughter --- to measure nuclear reactions

Activity:

growth of - . . . . . . . . . . . . . 13

half-life by direct measurement of specıfıc -- . . . 103

measurement of half-lıfe by specıfic - - . . . 103

Algebra

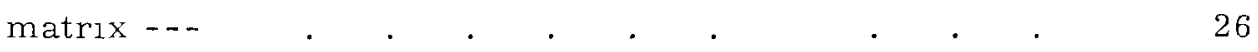

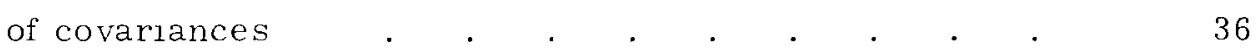

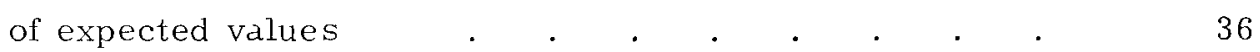

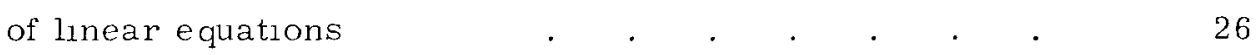

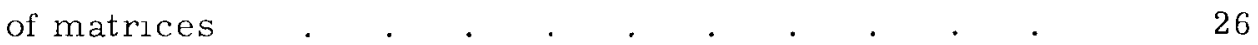

of variances . . . . . . . . . . . . . . . 36

Amplitude . . . . . . . . . . . . . . . 124

Analysis

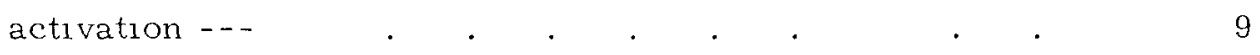

improper use of least-squares -- . . . . . . . . . 94

of replicate samples $\quad . \quad$. $\quad . \quad . \quad . \quad 35,95$

of varrance $\quad$. . . . . . . . . . . 35,95

statistical imprecision of a single radiochemical --- 95

Analytıcal

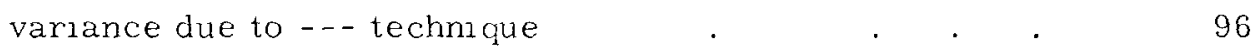

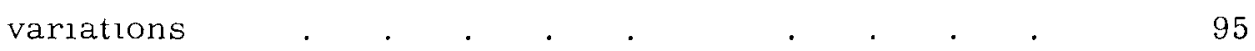


Page No.

Arithmetic average . . . . . . . . . . . . . 40

Assumptions underlying decay equations . . . . . . . . 3

Average . . . . . . . . . . . . . . . . . 31

arıthmetic - - . . . . . . . . . . . 40

best - - value for a given number of replicates . . 98

best value of an - . . . . . . . . . . . . 46

estimated measure of the precision of the -.. of a number of measurements . . . . . . . . . 42

having the least variance . . . . . . . . . 45

individual quantities which are to be --- are not uncorrelated . . . . . . . . . . 45

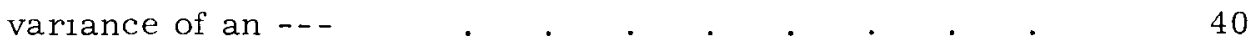

variance of a weighted - - . . . . . . . . . . . . 47

variance of best - _- value for a given number of replicates 98

welghted -. . . . . . . . . . . . . 4 44,45

weighting factor to -.- the replicate results . . . . 96

Averaging

and sampling statıstıcs . . . . . . . . . . 39

measurements of unlike variances . . . . . . . 45

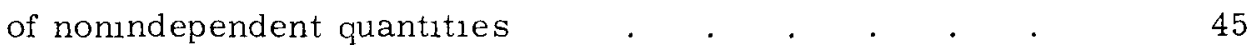

replicate values . . . . . . . . . . . . . 98

werght in the ..- process . . . . . . . 44

Avoldance of computer overflow . . . 94

Avolding: $\quad . \quad$. . . . . . . . . . . . . . . . . . . 94

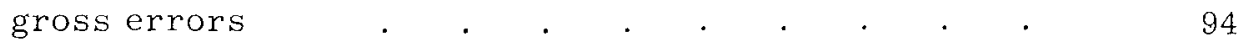

losses in slow circuit components . $\quad . \quad . \quad . \quad . \quad 117$

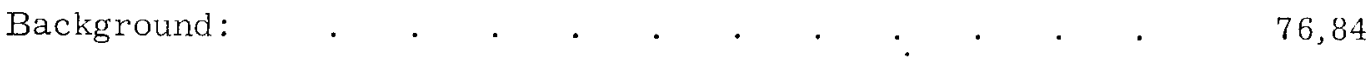

correction for counter -. . . . . . . . . . . . 76

correction when Poisson distribution is not applicable . 76

nonrandom . . . . . . . . . . 76

renormalızed probability distrıbution of --- count 76 
Bernoulli distribution

expected value of

expected value of a count from observed count for ---

standard deviation of ...

varıance of expected value of a count for -.. . .

Blocking of a counter

Branched decay

Branching

decay

ratio

growth factor in --- decay

Buildup toward equilibrium

Cascade

Chains

decay --- with more than two consecutive decays . .

\section{Chance}

coincident events

ratio of true to ... coincidences

Ch1-square test

Ch1-squared

test of goodness of fit

Circuit

avording losses in slow -.- components

distribution of intervals between output pulses from a scale-of -m -..

electronic scaling ---

regularizing action of a scaling -.-

scaling -- -

Coefficients

Cofactor of a matrıx element

Colncidence

counting of large solid angles . . . . . . . . . . 122

expected value of the false - - rate . . . . . . 123

false - . . . . . . . . . . . 125,126 
Colncidence:

Page No.

measurements of absolute counting efficiency by -.techniques

precision of absolute counting by ---

ratio of true to chance -..

true

Councident

chance -- events . . . . . . . .

effects of - - events on discriminated counts . . . 124

events . . . . . . . . . . . . . . . 124

measurement of $\ldots$ events . . . . . . . 120

true -- counts . . . . . . . . . . . 125

true versus false -- counts . . . . . . . 122

truly -- events . . . . . . . . . . 120

varıance of -.- event rate . . . . . . . . . . . . 123

varıance of the false - - rate . . . . . . . 123

Column vector . . . . . . . . . . . . . . . . . . 27

Combination.

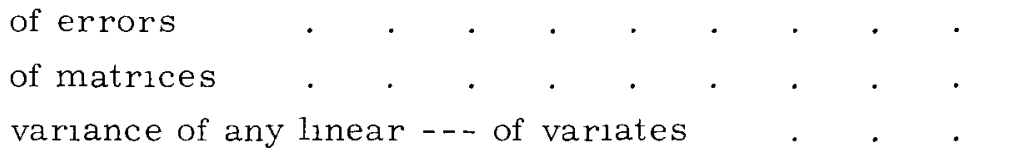

Complex systems.

mathematical techniques for derıving formulas for decay of

Component

precision of the amount of each - - present . . . . 87

\section{Components}

avolding losses in slow circuit - - . . . . . . . . 117

correction for presence of daughter in parent-daughter systems where both -.- have comparable half-lives

F-test for number of -. . . . . . . . . . 60

identification of.$- \quad$. . . . . . . . . . . . . 101

number of - . . . . . . . . . . . . 101

two or more long-lived -.. . . . . . . . . 107 
Page No.

Computational: least-squares --- method . . . . . . 61

Computer overflow: avoldance of --- . $\quad . \quad$. . . 94

Confidence:

$$
\begin{aligned}
& \text { limits . . . } \quad . \quad \text {. } \quad . \quad . \quad 33,34 \\
& \text { n-percent _.- interval . . . . . . . . . } 33
\end{aligned}
$$

Cons ecutive:

decay chains with more than two--- decays . . . . 21

decay for parent and daughter with equal half-life $\quad 11$

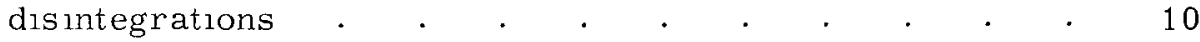

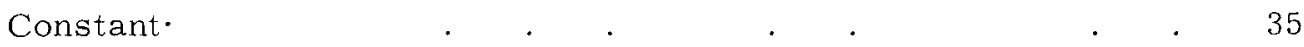

decay -.

method of evaluating a decay -- $\quad$. . . . 101

Constants:

partıal decay -- . . . . . . . . . . . . . . 101

Correction:

actual --- for dead time . . . . . . . . 113

background --- when Poisson distribution is not applicable. 76

factor for presence of daughter . . . . . . 12

for counter background . . . . . . . . . 76

for presence of daughtex in parent-daughter systems where both components have comparable half-lives . . . 13

for presence of daughter in parent-daughter systems where daughter is considerably longer-lived than parent . . 13

Correlation

Count:

distribution of expected values for a given observed -.-

for a large sampling . $\cdot$.

distribution of expected values for a given observed -.-

for a small sampling . . . . . . . . . . 68

expected value of a --- from observed --- for Bernoulli

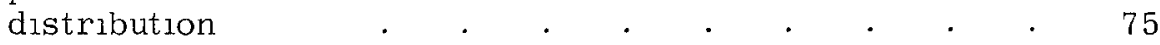

expected value of a -.. from observed value for Poisson

distribution 
Page No.

Count

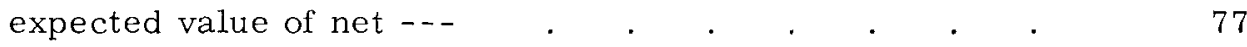

gross - . . . . . . . . . . . . . . 84

high - . . . . . . . . . . . . . .

probability of disintegrating and producing a --- . . . 64

renormalized probability distribution of background --- 76

varıance of an extrapolated -. rate . . . . . 79

varıance of an observed -- _ . . . . . . . . 84

varıance of expected value of a -.- for Bernoull

variance of expected value of - - for a Poisson

varıance of expected value of net -- . . . . . . . 76

Counter.

blockung of a

correction for -.- background . . . . . . $\quad 76$

measurement of --- dead time with an oscilloscope 118

measuring --- dead time . . . . . . . 114

saturation of a . . . . . . . . . . 113

Counting

additıve varıance due to --- equipment . . . . . . 86

coincidence -.. of large solıd angles . . . . 122

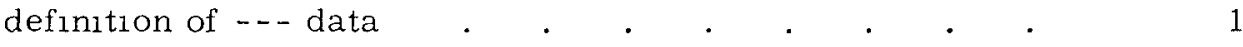

differential -. . . . . . . . . . . . . . 107

distribution functions of - - results . . . . . . 64

effect of dead time on observed -- rate . . . . . 112

efficiency . . . . . . . . . . . . . 64

geometry of --- arrangement . . . . . . . 65

maximum -. rate . . . . . . . . 113

measurement of absolute -- efficiency by colncidence

technques . . . . . . . . . . . . 127

net -- rate . . . . . . . . . . . . . 84

precision of absolute -- by coincidence $\quad . \quad$. $\quad 127$ 
Page No.

Counting.

probability that precisely $n$ counts will be recorded

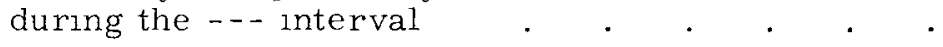

rate

single photopeaks . . . . . . . . . .

statıstics of ---data . . . . . . . . . . . . . . . 64

total -. - rate of a sample . . . . . . . . 11

variance of net -- rate . . . . . . . . . 84

Counts

effect of coincident events on discriminated - . . . . . 124

extrapolating -.- per unit tıme . . . . . . . 81

probability that precisely $n$-.- will be recorded during

the counting interval . . . . . . . .

true colncident - - . . . . . . . . . . . 125

true versus false coincident -.- . . . 122

Covariance

diagonal elements of --- matrıx are varıances

matrıx

of $x$ and $y$

Covariances

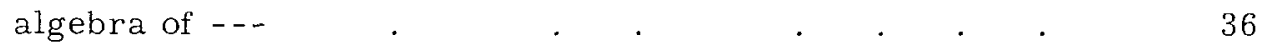

Criteria for rejection of data $\quad . \quad$. . . . . . . . . 92

Criterion additional -.- for rejecting data . . . . 93

Cross section reaction --- measurement . . . 83

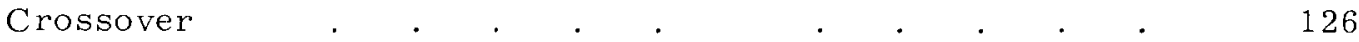

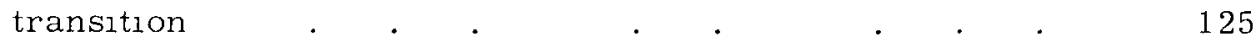

Cutoff energy - - . . . . . . . . . . . . . . . . $\quad 87$

Daughter. $\quad . \quad$. $\quad . \quad$. . . . . . . 10

analytıcal scheme when both parent and -.- are formed directly by nuclear reactions . . . . . . . . . 17

analytical scheme when parent is much shorter-lived than -- - 
Daughter

analytical scheme when both parent and -- - are formed directly by nuclear reactions

analytical scherne when parent is much shorter-lived than -.-

analytical schemes when half-life of parent is much longer than that of -.-

consecutive decay for parent and -.- with equal half-life

correction for presence of --- in parent-daughter systems where both components have comparable half-lives

correction for presence of -- in parent-daughter systems where - - is considerably longer-lived than parent

use of -.. activities to measure nuclear reactions

Dead time

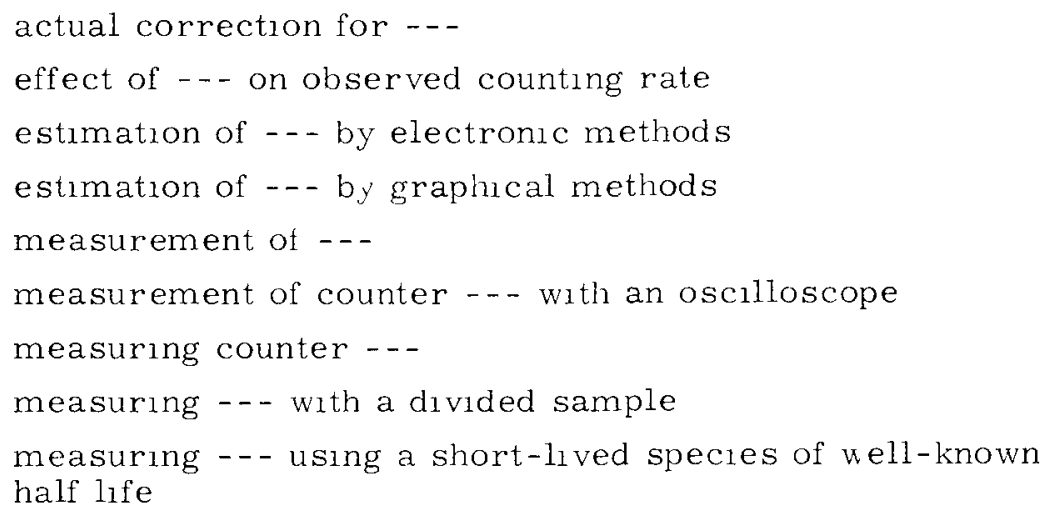

measurement of counter -.. with an oscilloscope

measuring counter ---

measuring - - with a divided sample

measurıng - - using a short-11ved species of well-known half life

Decay

assumptions underlyıng --- ecuations

branched -.-

branching -..

chains with more than two consecutive ---

comparison of the --- of the unknown species with that of

a species with precisely known half-life

consecutive - - for parent and daughter with equal halflife

constant

curve

curve of a two-component system

formation of active species by -.- of a radioactive precursor 
Decay

Page No.

fundamental law of radroactive - - . . . . . . 3

graphical resolution of --- curve . . . . . . . 6

growth factor in branching -- - . . 20

least-squares solution to resolve --- data . 83

mathematical techniques for deriving formulas for --of complex systems . . . 21-24

matrix inversion method for resolution of - - curve . 87

method of evaluating a -- constant 101

observing --- over long periods of time . 103

of a parent-daughter pair where parent is the longerlived species

of a parent-daughter pair where parent is the shorterlived species . . . . . . . 14

of a parent-daughter system . . . . . . . . 12

partial -. constants . . . . . . . . 19

proper weighting factors for use in a least-squares

calculation of -.- data $\quad$. $\quad . \quad$. . . . 87

proper weighting of --- data for a least-squares solution 83

rate for the false sum peak . . . . . . 127

resolution of --- data . . . . . . . . . 83

resolving the -- data . . . . . . . 4

scheme . . . . . . . . . . . 65

two-component -- curve . . . . . . 6

Decays.

self-calıbrating method for measuring long-lived --- . 105

Definition of counting data . . . . . . . . 1

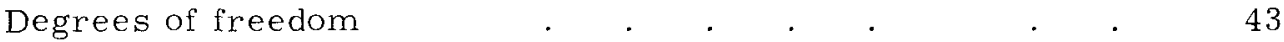

Depleted target material _ . . . . . $\quad$. 9

Destruction of product . . . . . . . . . $\quad$. 10

Detection of multicomponent systems . . . 105

Detector efficiency $\quad$. $\quad$. . . . $\quad$. 9

Determinant of a square matrix . . . . . . . . . . . $\quad$. 29

Deviation:

standard - - of Bernoulli distribution . 66

standard - - of Poisson distribution . . . 67

standard - - of normal distribution . . . 68

standard - - of $\mathrm{x} . . .433$ 
Page No.

Deviations

number of standard - - . . . . . . . . . . . . $\quad$. 92

Diagonal.

elements of covariance matrix are variances . . . 87

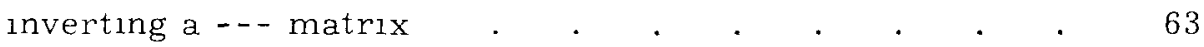

$\operatorname{matrix} \quad . \quad . \quad . \quad . \quad . \quad . \quad . \quad . \quad 29$

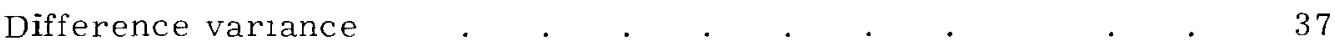

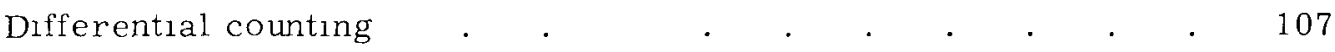

Discriminated.

effect of coincident events on --- counts . . . . 124

Disintegrating

probability of -- - and producing a count 64

Disintegration.

measurement of true -- rate . . . . . . 127

Disintegrations.

consecutive

Distribution

background correction when Poisson -.- is not applicable

Bernoull _. . . . . . . . . . . 65

expected value of a count from observed count for

Bernoull _.. . . . . . . . 75

expected value of a count from observed value for a

Polsson -. . . . . . . . . . . . 69

expected value of Bernoulli - - . . . . . . . . . 65

function . . . . . . . . . . . . . $31-33$

functions of counting results . . . . . . . . 64

Gaussian - - . . . . . . . . . . . . . . 67

normal -. . . . . . . . . . . . . 67

normalized - - function . . . . . . . . . . . . 32

of expected values for a given observed count for a

large sampling . . . . . . . . 69

of expected values for a given observed count for a small sampling . . . . 68

of intervals between output pulses from a scale-of-m
circuit
. 
Page No.

Distribution.

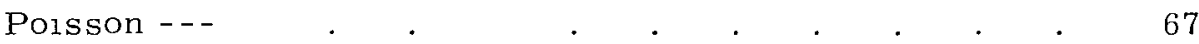

Polsson - - not valid . . . . . . . . 70

renormalızed probability - - of background count . . 76

standard deviation of normal - - . . . . . . . . $\quad$. 68

standard deviation of Bernoull _.. . . . . . . 66

standard deviation of Polsson - .- . . . . 67

variance of a total - - . . . 42

variance of expected value of a count for a Poisson -- . $\quad 69$

variance of expected value of a count for Bernoull _._ . 75

variance of the --- formed by a sample . . . . 42

Divided sample

measuring dead time with a $\ldots . . . \quad . \quad . \quad$. 114

Efficiency.

countıng - . . . . . . . . . . . . . 64

detector . . . . . . . . . . . . . . . . . . . . . 9

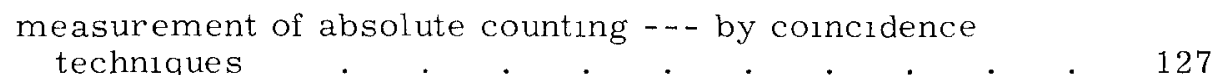

Electronic

estimation of dead time by --- methods . . . . 116

scaling circuit . . . . . . . . . . . . . 117

Element.

cofactor of a matrix _. . . . . . . . . . . . . . 29

of the matrix . . . . . . . . . . . 27

Elements

diagonal -. - of covariance matrix are varıances . . 87

Energy

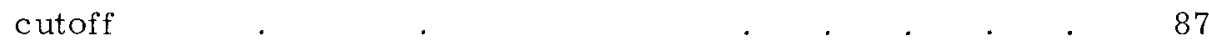

particle - - spectra . . . . . . 124

Equation

inverse of matrix of the normal - - . . . . 52

inverse matrıx of the normal - - . . . . . . . 61

number of terms in the $\ldots$. . . . 60 
Page No.

Equations

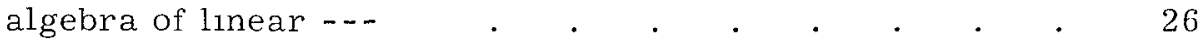

assumptions underlyıng decay $\quad . \quad$. $\quad . \quad$. . . 3

normal -. $\quad . \quad . \quad . \quad . \quad . \quad . \quad . \quad . \quad . \quad .49,52,89$

normal - - in matrix notation . . . . . . . . . 49

simultaneous linear -- . . . . . . . . . . . . 26

weighted -.- for data in matrix form . . . . . . 61,62

Equilibrium

bulldup toward - . . . . . . . . .

Equipment

additive variance due to counting

Estimate.

best - . . . . . . . . . . . . .

best - - of the true value for an experiment . . 96

graphical - - of the half-1ife . . 6

of the half-lives $\quad . \quad$. $\quad . \quad . \quad . \quad . \quad . \quad . \quad 5$

to correct for variances not included in least-squares

unbiased - - of variance . . . . . . . 42

unbiased - - of variance in least-squares solution . . 56

unbiased --- of varıance of points in least-squares solution 53

Estimated.

measure of the precision of an individual measurement .

measure of the precision of the average of a number of measurements

Estimation

of dead tıme by electronic methods . . . . . . 116

of dead time by graphical methods . . . . . . 116

of instrumental variance . . . . . . 88

of population variance from a sample . . . . . . 41

of variance due to instrumental variation $\quad$. $\quad 87$

precision of - - of a mean . . 40

Evaluating

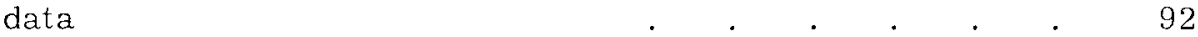

1teration methods for --- lambda . . . 107

method of -- a decay constant . 101 
Page No.

Evaluation

graphical -.- of lambda . . . . . . . . . . . 102

of lambda for known multicomponent systems . . . 107

Event rate.

variance of coincident

Events

chance coincident -.. $\quad . \quad . \quad . \quad . \quad . \quad . \quad . \quad . \quad 120$

colncident -. . . . . . . . . . . 124

effect of coincident - - on discriminated counts . . 124

measurement of coincident - - . . . . . . 120

probability of observing $\mathrm{N}_{-}-$. $_{0}$. . . . . 66

probability of recording exactly $\mathrm{N} \ldots$. . . . bu

truly coincident . . . . . . $\quad$. 120

Fxpansion by minors . . . . . . . . . . . 29

Expected value.

algebra of - - . . . . 36

distribution of -.- for a given observed count for a large sampling

distribution of -.. for a given observed count for a small sampling

of a count from observed count for Bernoulli distribution . $\quad 75$

of a count from observed value for a Poisson distribution . 69

of a matrix or vector . . . . . . . . 52

of any function of $\mathrm{x}$. $\quad . \quad$. . . . . . . . 32

of Bernoulli distribution . . . . . . . . . . 65

of $\mathrm{n}$ and its variance where we have an actual observed

value . . . . . . . . . . 68

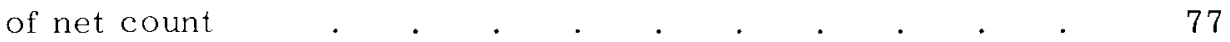

of the falsc colncidence rate . . . . . . . . . 123

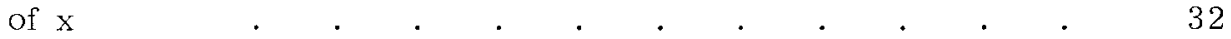

variance of - - of net count . . . . . . . . . . 77

variance of --- of a count for a Bernouli distribution . 75

variance of --- of a count for a Poisson distribution . . 69 
Experiment:

best estimate of the true value for an -.. . . . . . 96

information to be obtained in an --- on radioactive

materıal . . . . . . . . . . . 4

Extrapolated

variance of an -.- count rate . . . . . . . 79

Extrapolatıng

counts per unit of time $\quad . \quad$. $\quad . \quad . \quad . \quad . \quad . \quad 81$

Extrapolation.

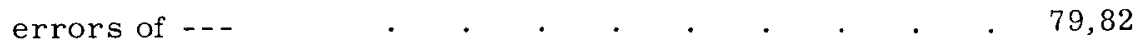

factor . . . . . . . . . . . . . . . . . . . . . . . . . .

variance . . . . . . . . . . . . $\quad .82$

variance of the -- factor . . . . . . . . 81

F-test for number of components $\quad . \quad$. $\quad . \quad 6 \quad$. $\quad$. 60

Figure

loss

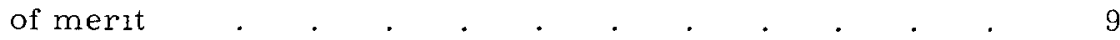

Figures

significant

Fit.

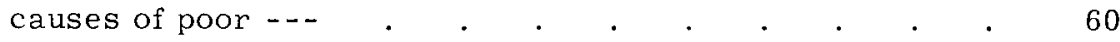

chr-squared test of goodness of -- . . . . . . 60

effect of varyıng an input lambda on the -..- of the data to

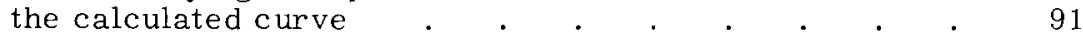

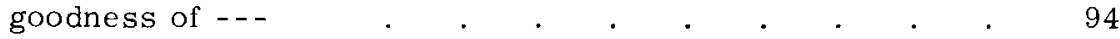

Fluctuations.

variance due to statistical - - $\quad . \quad \ldots \quad . \quad . \quad . \quad . \quad 96$

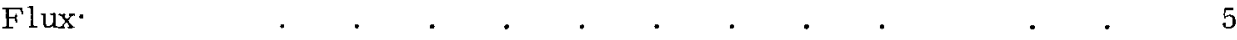

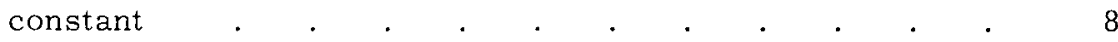

reactor -.- measurement . . . . . . . . 83

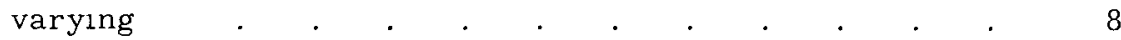

Form

improper --- for function being fitted . . . . . . $\quad .94$

Formation

independent -. $\quad$. $\quad . \quad$. $\quad$. 21

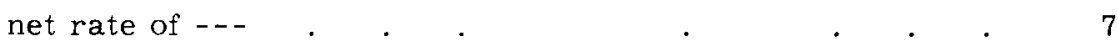

of active species . . . . . . . . . . . . $8-10$

of active species by decay of a radioactive precursor . 10

of species in nuclear reactions $\quad$. $\quad . \quad$. 5 
Formed:

$\underline{\text { Page No }}$

analytical scheme when both parent and daughter are ---

directly by nuclear reactions

variance of the distribution --- by a sample . . . 42

Formulas.

mathematical techniques for derıving -.- for decay of complex systems . . . . . . . . . 21-24

Freedom.

degrees $. \quad . \quad . \quad . \quad . \quad . \quad . \quad . \quad . \quad . \quad 43$

Function

distribution . . . . . . . . . . 31-33

improper form for -.- being fitted . . . . . 94

expected value of any -- of $\mathrm{x} \quad . \quad . \quad . \quad . \quad 32$

normalized distribution --

Functions

distribution --- of counting results . . . . . 64

Fundamental.

law of radioactivity $. \quad . \quad . \quad . \quad . \quad . \quad . \quad . \quad . \quad 103$

law of radioactive decay . . . . . . . . . . 3

Gamma spectrum.

difficulty in interpreting sum peaks in -- . . . . . 126

Gaussian distribution $\quad . \quad$. $\quad . \quad$. . . . . . . . . . . 67

Geometry:

of counting arrangement . . . . . . . . . . 65

Goodness of fit: . . . . . . . . . . . . 94

ch1-squared test of -. $\quad . \quad . \quad . \quad . \quad . \quad .60$

Graphical.

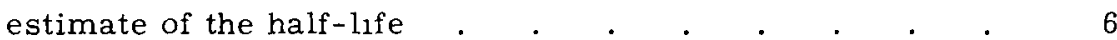

estimation of dead time by --- methods . . . . . 116

evaluation of lambda $\quad . \quad . \quad . \quad . \quad . \quad . \quad . \quad . \quad 102$

resolution of decay curve $\quad . \quad \ldots \quad . \quad . \quad . \quad . \quad 6$

Growth

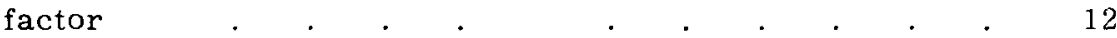

factor in branching decay . . . . . 20

of activity . . . . . . . . . . . .

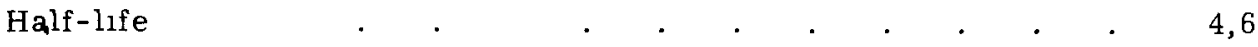

alteration in the -.-value . . . . . . 91

analytical schemes when --- of parent is much longer than that of daughter $\quad . \quad . \quad . \quad . \quad . \quad . \quad . \quad 15$ 
Page No.

Half-life:

by direct measurement of specific activity . . . 103

consecutive decay for parent and daughter with equal - - . 11

effect of an impurity on the measurement of a -- . . . 101

least-squares solution for - - . . . . . . . 102

measurement of a --- in a single-component system of

short or moderate -- . . . . . . . 102

measurement of --- in a single-component system with

long - . . . . . . . . . . . 103

measurement of - - by specific activity . . . . 103

measuring dead time using a short-lıved species of wellknown -. . . . . . . . . 115

moderate - . . . . . . . . . . 102

precisely known . . . . . . . . . . 103

Half-lives

correction for presence of daughter in a parent-daughter

systems where both components have comparable ... .

difficulty of resolving two species with similar -. . . 106

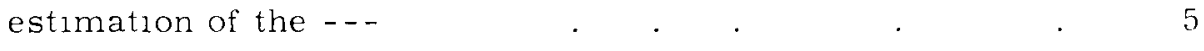

measurement of - . . . . . . . . . . 101

partial - . . . . . . . . . . . . 20

sensitivity of $\mathrm{x}$ to the input - - . . . . . . . . . . 95

Half-period . . . . . . . . . . . . . . . . . . . 4

High count rates . . . . . . . . . . . . . . 113

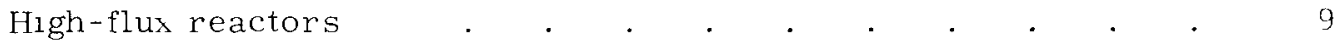

Human error . . . . . . . . . . . . . . . . . . . 92

Identification of components . . . . . . . . . . . . 101

Imprecision*

statistical - - of a single radiochemical analysis . . 95

Improper:

form for function being fitted . . . . . . . . 94

use of least-squares analysis . . . . . . . . . . 94

Improving.

values of the input lambda $\quad . \quad$. $\quad . \quad$. $\quad . \quad 92$

Impurities

Impurity

effect of an --- on the measurement of a half-life 
Page No.

Independent formation

Indıvidual.

estimated measure of the precision of an - - measurement 42 quantities which are to be averaged are not uncorrelated. 45 varıance of an --- measurement . . . . . . 40

Information.

loss of --- due to rounding errors

to be obtained in an experiment on radioactive material . 47

Instrument malfunction

Instrumental variance.

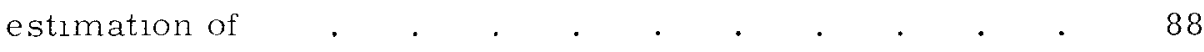

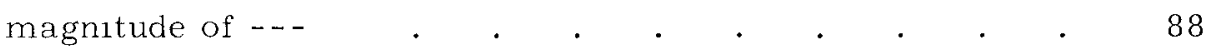

Instrumental variations.

estimation of variance due to -. . . . . . . . . . . 87

Integral.

treatment of unknown system by --- transforms . . 110

Intensity

sum peak

Interpolation

variance due to neglect of --- lights . . . . . . . 85,86 Interpreting.

difficulty in -.. sum peaks in gamma spectra . . . 126

Interval

average - - between pulses . . . . . . 117

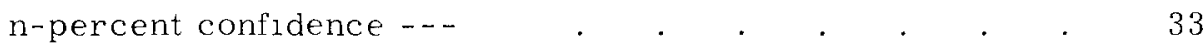

Intervals

distribution of --- between output pulses from a scale-of-m circuit

Inverse.

matrix 29,30

matrix of the normal equation

of matrix of the normal equation

Inversion

matrix -- by orthogonalizing . . . . . . . 61

matrix --- method for resolution of decay curve $\quad . \quad$. 87 
Page No.

Inverting

a diagonal matrix $. \quad . \quad . \quad . \quad . \quad . \quad . \quad . \quad 63$

Ionization.

measurement of half-life using two --- chambers . 104

Iteration.

methods for evaluating lambda . . . . . . 107

Lambda.

effect of varying an input --- on the fit of the data to the calculated curve

evaluation of --- for known multicomponent systems . . 107

graphical evaluation of - - . . . . . . . . 102

improving the values of the input - - . . . . . . . . 92

iteration methods for evaluating -. . . . . . . . 107

measurement of.$--\quad . \quad . \quad . \quad . \quad . \quad . \quad 101$

Lambdas:

effect of a slight change in one of the --- resolution . .

systematic effect on resolution caused by slight variations in the values of the -. . . . . . . .

Law:

fundamental - - of radioactive decay . . . . . . 3

fundamental --- of radioactivity . . . . . . 103

Least-squares.

computational method . . . . . . . . . 61

effect on --- solutions of any unanticıpated variances . 83

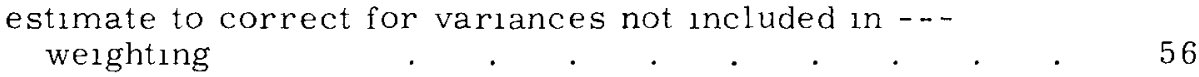

improper use of -.- analysis . . . . . . . . . $\quad .94$

precision of each parameter in a - - solution . . 56

proper weighting factor for use in a --- calculation of

decay data . . . . . . . . 87

proper weighting for -- . . . . . 50

proper weighting of decay data for a --- solution . 83

Least-squares solution.

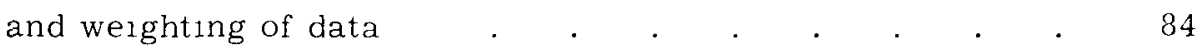

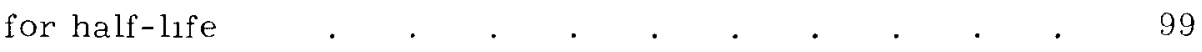


Page No.

Least-squares solution:

in matrix notation . . . . . . . . . . 50

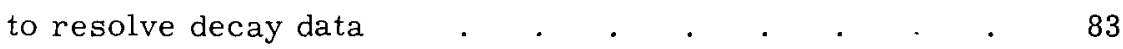

unbiased estimate of variance in -. . . . . . . . 56

unbiased estimate of variance of points in -.. . . . . 53

variances of results of -. . . . . . . . . . 52

weighting factors in - . . . . . . . . . . . 48

Limits:

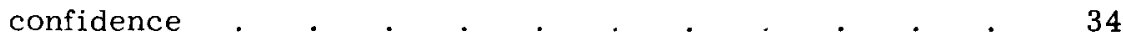

Linear:

algebra of -.- equations . . . . . . . . . . . 26

simultaneous --- equations . . . . . . . . 26

variance of any --- combination of variates . . . 39

Live time . . . . . . . . . . . . . . . 117

Long-lived:

self-calibrating method for measuring --- decays . . 105

two or more --- components . . . . . . . 107

Losses:

avoiding -- in slow circuit components . . . . 117

-- - due to slow register response . . . . . 117

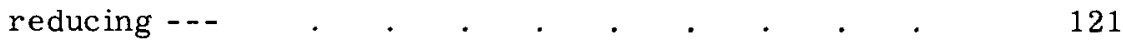

Magnitude:

instrumental variance $\quad . \quad . \quad . \quad . \quad . \quad . \quad . \quad . \quad 88$

Malfunction:

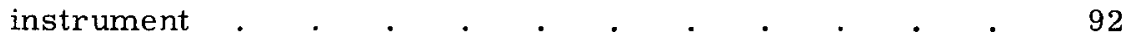

Mathematical:

techniques . . . . . . . . . . . . . . . 25

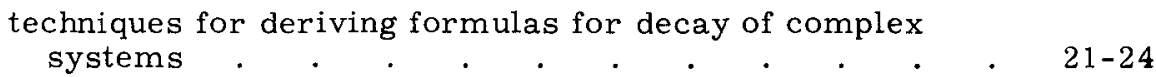

Mathematics:

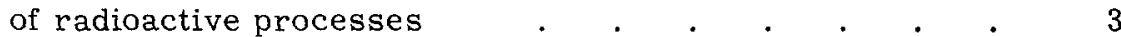

statistical . . . . . . . . . . . . 31

Matrices:

algebra of -- . . . . . . . . . . . . . 26

combination of - . $\quad . \quad . \quad . \quad . \quad . \quad . \quad . \quad . \quad 27$

multiplication of.$--\quad . \quad . \quad . \quad . \quad . \quad 28$ 


\section{Page No.}

Matrix

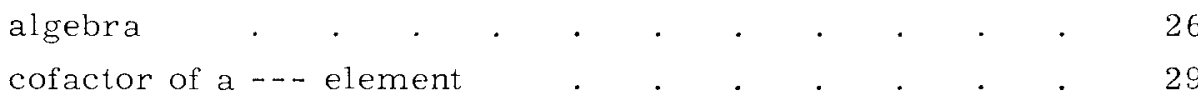

covariance

$39,46,52$

determinant of a square - . . . . . . . . . . . . 29

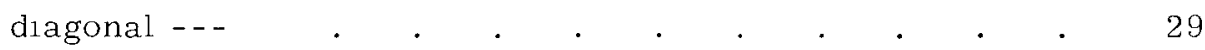

diagonal elements of covariance --- are variances . . 87

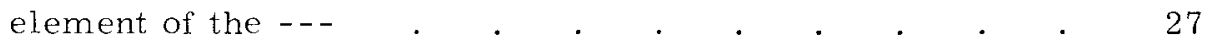

expected value of a --- or vector . . . . . . . 52

inverse . . . . . . . . . . . . . 29,30

inverse - - of the normal equation . . . . . . . 61

inverse of - - of the normal equation . . . . . . 52

inversion by orthogonalızing . . . . . . . . . 61

inversion method for resolution of decay curve $\quad$. $\quad 87$

inverting a diagonal - - . . . . . . . . 63

least-squares solution in - - notation . . . . 50

lower triangular ... . . . . . . . . . . . 29

multiplication . . . . . . . . . . 28

nonsingular - - . . . . . . . . . . . 29

normal equations in -- notation . . . . . . 49

orthogonalization process . . . . . . . . . . 62

relationships . . . . . . . . . . . 30

singular -. $\quad$. $\quad . \quad$. . . . . . . . . 29

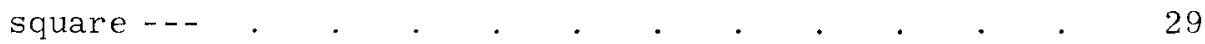

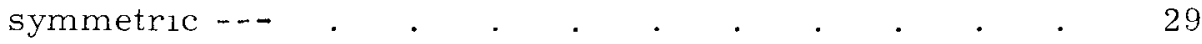

transpose of a $\ldots$. . . . . . . . . . . . . 527

upper triangular - - . . . . . . . . . . . . . 29

unit - - . . . . . . . . . . . . . . 29

weighted equations for data in --- form . . . . 61,62

zero-- . . . . . . . . . . . . . . . 29

Matrix-vector.

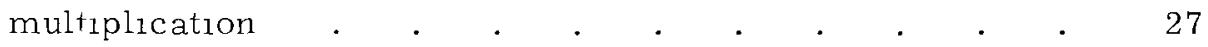

notation $. \quad . \quad . \quad . \quad . \quad . \quad . \quad . \quad . \quad . \quad . \quad 26$ 
Page No.

Maximum.

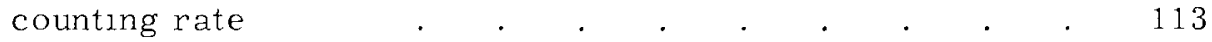

production of a radioactive species . . . . . . 10

Measure

estimated --- of the precision of an individual measurement

estimated -.. of the precision of the average of a number of measurements

Measured

probability that a -- value of $\mathrm{x}$ will fall between $\mathrm{A}$ and $\mathrm{B}$.

Measurement.

effect of an impurity on the - - of a half-life . . . 101

estimated measure of the precision of an individual --- . 42

half-lıfe by direct --- of specıfic actıvity . . . . 103

of a half-life in a single-component system of short or

moderate half-life . . . . . . . . 102

of absolute counting efficiency by coincidence techniques . 127

of coincident events . . . . . . . . . . 120

of counter dead time with an oscilloscope . . . . 118

of dead time . . . . . . . . . . . . 114

of half-lives . . . . . . . . . . . . . . . 101

of half-life by specific activity . . . . . . 103

of half-life in a single-component system with long half-

life.......... . . 103

of half-life using two lonization chambers . . . . 104

of lambda . . . . . . . . . . . . . 101

of true disintegration rate . . . . . . . . . 127

reaction cross section -.. . . . . . . . . . . 83

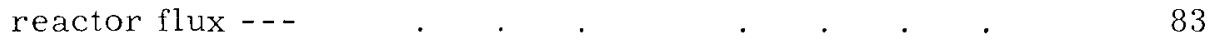

variance of an individual -. . . . . . . . 40

Measurements.

averaging - - of unlike variances . . . . . . 45

estimated measure of the precision of the average of a

statıstical reliability of - - on nuclear reactions . . 82 
Page No.

Measuring*

counter dead time . . . . . . . . . . . . 114

dead time using a short-lived species of well-known halflife . . . . . . 115

dead time with a divided sample . . . . 114

Mechanical registers

Merit .

figure of ---

Minors.

expansion by ---

moderate half-life

Multicomponent

detection of --- systems

evaluation of lambda for known --- systems

unknown --- systems

Multiplication

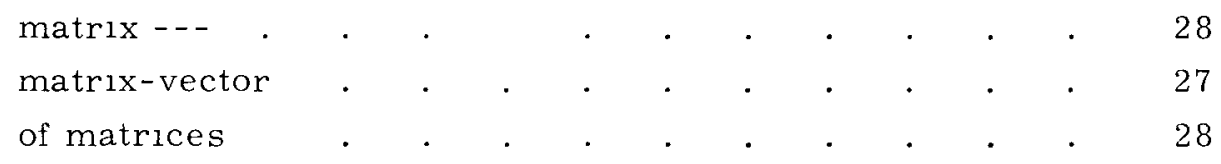

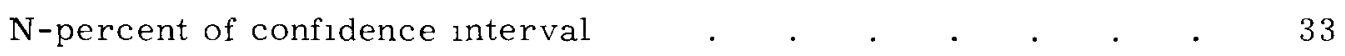

Neglect

variance due to --- of interpolation lights . . . . . 85,86

Nonindependent averaging of --- quantities . . . . . . . 45

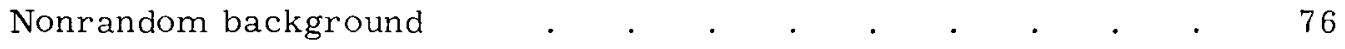

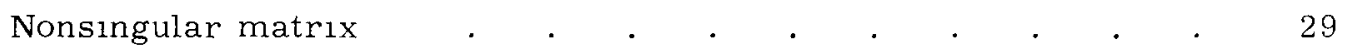

Normal.

distribution

67

equations

equations in matrix notation

$49,52,89$

inverse matrix of the --- equation

49

inverse of matrix of the --- equation . . 52

standard deviation of - - distribution . 68

Normalized distribution function 
Page No.

Notation

least-squares solution in matrix -- $\quad$. . . . 5

matrix-vector -. . . . . . . . 26

normal equations in matrix -.. $\quad . \quad$. $\quad . \quad$. . . . 49

Nuclear reaction

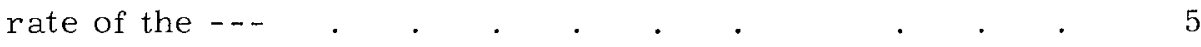

Nuclear reactions.

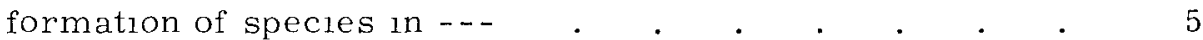

statistical reliability of measurements on --- . . . . 82

use of daughter activities to measure -. . . . . . . 13

Number

F-test for -- of components . . . . . $\quad$. 60

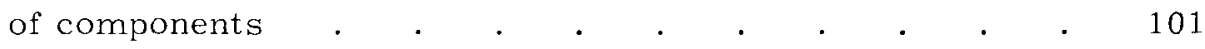

of standard deviations . . . . . . . . . . . . . 92

of terms in the equation . . . . . . . . . 60

variance of best average value for a given - - of replicates 98

Observed count

distribution of expected values for a given --- for a large sampling

distribution of expected values for a given --- for a small sampling $\quad$. . . . . . $\quad 68$

expected value of a count from --- for a Poisson distribution 69

expected value of a count from -..- for Bernoulli distribution 75

expected value of $\mathrm{n}$ and its variance where we have an actual ... of $\mathrm{n}$. . . . 68

variance of $---\quad . \quad . \quad . \quad . \quad . \quad . \quad . \quad 84$

Observed counting rate.

effect of dead time on -- $\quad$. $\quad . \quad . \quad . \quad . \quad . \quad . \quad 112$

Observing

decay over long perıods of time . . . . . . . $\quad . \quad 103$

probability of -.-n events . . . . . . . . . 66

Orthogonal vectors . . . . . . . . . . . . . . 30

Orthogonalization

matrix - . process . . . . . . . . . 62

Orthogonalizing

matrıx inversion by _.. . . . . . . . . . . . 61 
Page No.

Oscilloscope:

measurement of counter dead time with an --- . . . 118

Output:

data

distribution of intervals between --- pulses from a scaleof-m circuit

Overflow:

avoidance of computer ---

Parameter:

precision of each - - in a least-squares solution

Parameters:

effect of input --- on resolution . . . . . . . 89

Parent:

analytical scheme when both -.. and daughter are formed

directly by nuclear reactions

analytical scheme when --- is much shorter-lived than

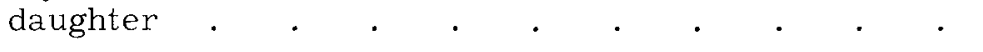

analytical schemes when half-life of --- is much longer than that of daughter . . . . . . .

consecutive decay for -.- and daughter with equal half-

life

Parent-daughter:

correction for presence of daughter in --- systems where both components have comparable half-lives

correction for presence of daughter in --- systems where daughter is considerably longer-lived than parent

decay of a --- pair where parent is the longer-lived species

decay of a -- pair where parent is the shorter-lived species

decay of a --- system

Partial:

decay constants

half-lives

Particle energy spectra

Pattern:

systematic --- in residuals 
Page No.

Percentage error

Period

Periods:

observing decay over long --- of time

Photopeaks:

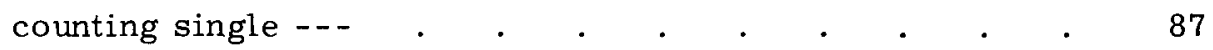

Points:

suspect

93,94

unbiased estimate of variance of -.- in least-squares solution

Poisson distribution:

background correction when --- is not applicable . . 76

distribution not valid . . . . . . . . 70

expected value of a count from observed value for a --- . 69

standard deviation of -. $\quad . \quad . \quad . \quad . \quad . \quad . \quad . \quad . \quad 67$

variance of expected value of a count for a -.. . . . . 69

Poor fit:

causes of.$\quad \cdot \quad \cdot \quad \cdot \quad \cdot \quad \cdot \quad \cdot \quad \cdot \quad \cdot \quad \cdot \quad \cdot 60$

Population:

estimation of --- variance from a sample . . . . 41

Precision:

estimated measure of the --- of an individual measurement . . . . . . . . . . .

estimated measure of the -.- of the average of a number of measurements

of absolute counting by coincidence . . . . . . 127

of each parameter in a least-squares solution . . . 56

of estimation of a mean . . . . . . . . . . 40

of the amount of each component present . . . . $\quad$. 87

Precursor:

formation of active species by decay of a radioactive -- . 10

Probability: . . . . . . . . . . . 13

of disintegrating and producing a count . . . . 64

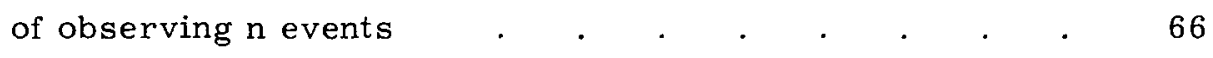

of recording exactly $\mathrm{n}$ events $\quad . \quad . \quad . \quad . \quad . \quad . \quad 665$ 
Page No.

Probability:

that a measured value of $\mathbf{x}$ will fall between $A$ and $B$. $\quad 32$

that precisely $n$ counts will be recorded during the counting interval $\cdot$..$\quad \cdot \quad \cdot \quad \cdot \quad \cdot \quad \cdot \quad 65$

renormalized --- distribution of background count . . 76

Product:

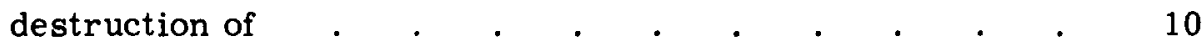

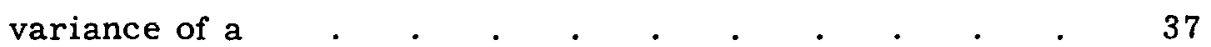

Production:

maximum --- of a radioactive species . . . . 10

Pulses:

average interval between --- . . . . . . . . . 117

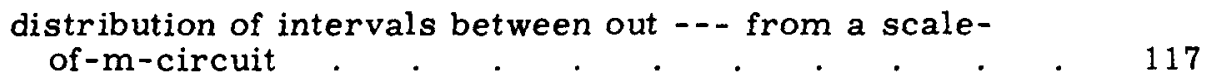

Quotient:

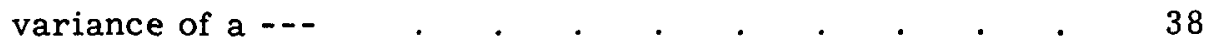

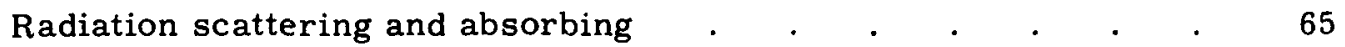

Radioactive:

formation of active species by decay of a --- precursor . 10

fundamental law of --- decay $\quad . \quad$. $\quad . \quad . \quad . \quad . \quad 3$

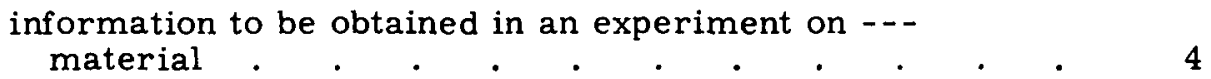

mathematics of --- processes . . . . . . . 3

maximum production of a --- species . . . . . 10

Radioactivity:

fundamental law of -. $\quad . \quad . \quad . \quad . \quad . \quad . \quad . \quad .103$

Radiochemical:

statistical imprecision of a single --- analysis $\quad$. $\quad 95$

Random errors . . . . . . . . . . . 25,31

Reaction:

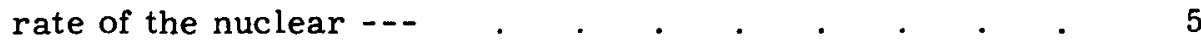

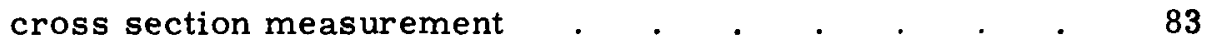

Reactions:

analytical scheme when both parent and daughter are

formed directly by nuclear -.. . . . . . . . 17

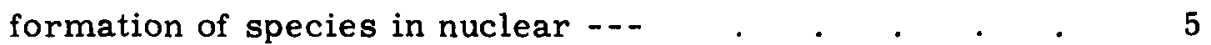


Page No.

Reactions:

statistical reliability of measurements on nuclear -.. . $\quad 82$

use of daughter activities to measure nuclear --- . . . 13

Reactor:

flux measurement ..$\quad$. . . . . . . 83

Reactors, high-flux . . . . . . . . . . . . . 99

Reducing, losses . . . . . . . . . . . . . 121

Reduction in rounding error . . . . . . . . . 63

Register:

losses due to slow -.- response . . . . . . . 117

Registers:

mechanical . . . . . . . . . . . 117

Regularizing:

action of a scaling circuit . . . . . . . . 117

action of scaling circuits $\quad . \quad . \quad . \quad . \quad . \quad . \quad . \quad 121$

Rejecting:

additional criterion for -.- data . . . . . . . . . 93

Rejection:

criteria for --- of data . . . . . . . . . . . 92

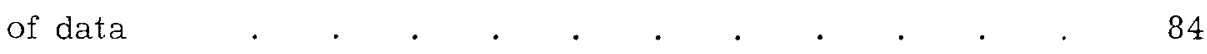

simple scheme for data -- . . . . . . . . . . 93

Reliability:

statistical -.- of measurements on nuclear reactions . 82

Renormalized probability distribution of background count . . 76

Replicate:

analysis of -- samples . . . . . . . . . 35,95

averaging - - values . . . . . . . . . . .

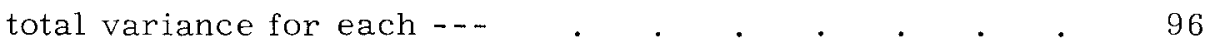

variance between --- samples . . . . . . . . . 996

weighting factor to average the -- results . . . $\quad 96$

Replicates: . . . . . . . . . . . . . . 95

best average value for a given number of --- . . . . . 98

variance of best average value for a given number of -.- . 98 
Page No.

Residual

welghted . . . . . . . . . . . . . . . 92

Residuals.

systematic pattern in - - . . . . . . . . . 94

weighted residuals of the system . . . . . . . 63

Resolution.

effect of a slight change in one of the lambdas on - . . . 89

effect of input parameters on -- . . . . . . . . 89

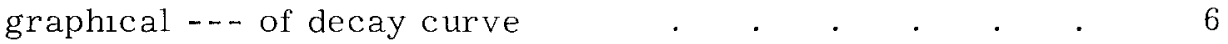

matrix inversion method for -.- of decay curve . . . 87

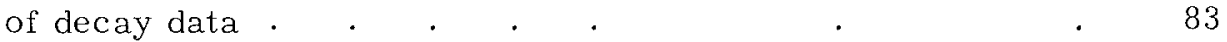

systematic effect on -.- caused by slight variations in

the values of the lambdas . . . . . 84

Resolve.

least-squares solution to --- decay data. . . . . 83

Resolving

difficulty of - - two species with similar half-lives . . 106

technique for -- data . . . . . . . . . 5

the decay data. . . . . . . . . . . . . 4

time . . . . . . . . . . . . . . . 112,120

Response

losses due to slow register - - . . . . . . . . 117

Rounding.

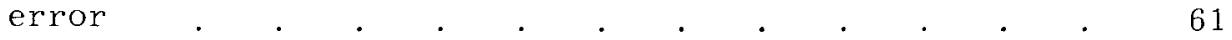

loss of information due to -.- errors . . . . 61

reduction in -- error . . . . . . . . . . . 63

Row vector . . . . . . . . . . . . . . . . 27

Sample

estimation of population variance from a -- . . . . 41

measuring dead time with a divided ... . . . . . 114

total counting rate of a - . . . . . . 11

variance of the distribution formed by a . . . . $\quad 42$ 
Page No.

Samples

analysis of replicate -. . . . . . . . . . 35,95

split . . . . . . . . . . . . . . . 114

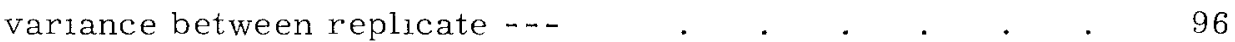

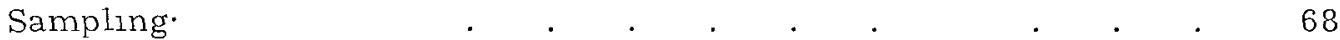

averaging and --- statistics . . . . . . . . . 39

distribution of expected values for a given observed

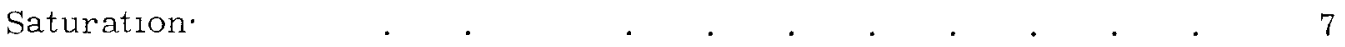

of a counter . . . . . . . . . . 113

Scale-of-m.

distribution of intervals between output pulses from a -.-

Scaling.

advantage to be gained by use of --- factors . . . 120

circuits . . . . . . . . . 85,117

electronic -. c c . . . . . . . . 117

regularizing action of a -- circult . . . . 117

regularızing action of.-- circuits . . . . . . 121

Scattering

radiation -.- and absorbing . . . . . . . . 65

Self-calibrating method for measuring long-lived decays . . 105

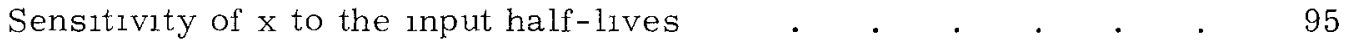

Significant figures . . . . . . . . . . . . . . . 61

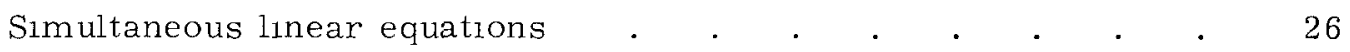

Single.

statistical imprecision of a --- radiochemical analysis . 95

Single-component.

measurement of a half-life in a --- system with long

half-life . . . . . . . 103

measurement of a half-life in a --- system of short

or moderate half-life . . . . . . . . 102

Singles

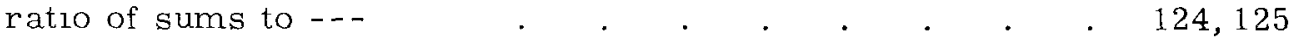


Page No.

Singular matrix

Solid

coincidence counting of large --- angles

Solution

least-squares --- and we1ghting of data . . 84

least-squares -- for half-life . . . . . 102

least-squares .. in matrix notation . . . 50

least-squares - - . . . . . . . . . . . . 47

least-squares - - to resolve decay data . . . . . 83

precision of each parameter in a least squares - - . . 56

proper weightıng of decay data for a least squares -- _ . 83

unbiased estımate of variance in least squares -.- . . . 56

unbiased estimate of variance of points in least squares - - 53

Solutions

effect on least squares --- of any unanticipated variances . 83

varıances of results of least squares - - . . . . . . 52

weighting factors in least squares - . . . . . . . 48

Species

difficulty of resolving two - - - with similar half-lives . 106

formation of active -. . . . . . . . . 8-10

formation of active -- by decay of a radioactive precursor $\quad 10$

formation of -- - in nuclear reactions . . . . . . 5

maximum production of a radioactive --- . . . . 10

measuring dead time using a short-lived --- of well-known

half-life . . . . . . . 15

Specific activity.

half-1 $\perp_{\perp}$ e by direct measurement of -- . . . . . 103

measurement of half-life by - - . . . . . . . 103

Spectra.

difficulty in interpreting sum peaks in gamma --- . . 126

particle energy - - . . . . . . . . . . 124

Split samples $\quad . \quad$. . . . . . . . . . . . . . 114 
Page No.

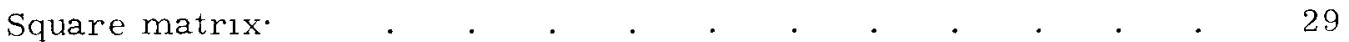

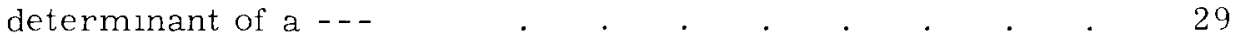

Squares.

welghted sum of the - - . . . . . . 49

Standard deviation

$\begin{array}{llll}\text { of Bernoulli distribution } & \text {. } & 66\end{array}$

of normal distribution . . . . . . . . . $\quad 68$

number of - - . $\quad$. $\quad$. $\quad$. $\quad . \quad 92$

of Poisson distribution. . . . 67

of $\mathrm{x} \quad$. $\quad . \quad$. 33

Statistical

imprecision of a single radiochemical analysis . . . . 95

mathematics . . . . . 31

reliability of measurements on nuclear reactions . $\quad 82$

variance due to --- fluctuations . . . . . . . 96

Statistics

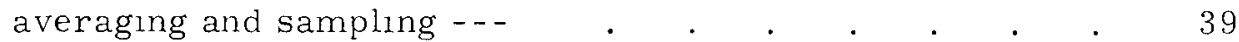

of counting data . . . . . . . . . . . . 64

substitution of a spurious zero-time . . . . . . . 94

Sum

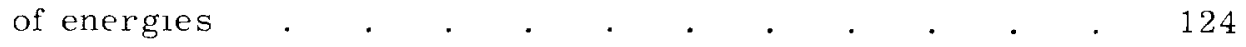

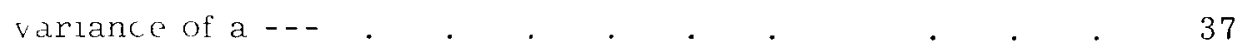

weighted -.. of the squares . . . . . . . 49

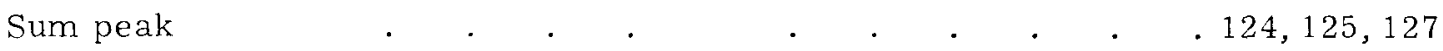

decay rate for the false - . . . . . . . . 127

difficulty in interpreting -.. in gamma spectra . . . 126

intensity of the - . . . . . . . . . 126

Sums

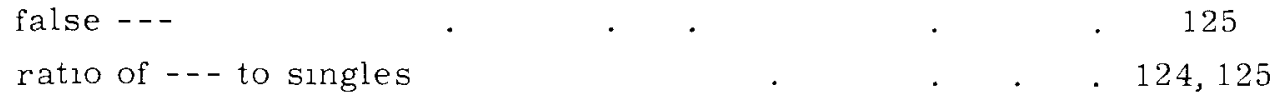

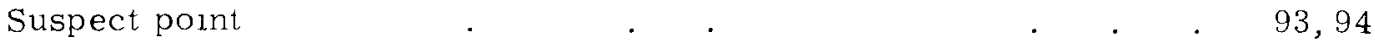

Symmetric matrix $\quad$. . . . . . . . 29 
Page No.

Systematic.

effect on resolution caused by slight variations in the values of the lambdas

errors

pattern in residuals

Target.

material depleted

Technique

for resolving data

variance due to analytical ---

Techniques

mathematical

mathematical -.- for deriving formulas for decay of complex systems

21,22

23,24

measurement of absolute counting efficiency by coincidence ---

Test.

ch1-square

ch1-square --- of goodness of fit

Time

actual correction for dead -..-

dead

$110,112,113$

effect of dead -- - on observed counting rate

112

estimation of dead - - by electronic methods

estımation of dead - - - by graphical methods

116

extrapolating counts per unit ---

live -- -

measurement of counter dead -.- with an oscilloscope

measurement of dead -..-

measuring counter dead -.-

measuring dead --- using a short-lived species of wellknown half-11fe

measuirng dead -.- with a divided sample

observing decay over long periods of -..

resolving -.. . 
Page No.

Transforms

treatment of unknown system by integral -..- .

Transition.

cross over . . . . . . . . . . . .

Transpose

of a matrix . . . . . . . . . . . 27

of a vector . . . . . . . . . . . . . . . . 27

Triangular

lower -. matrix . . . . . . . . . . . .

upper -- matrix . . . . . . . . . . . . . 29

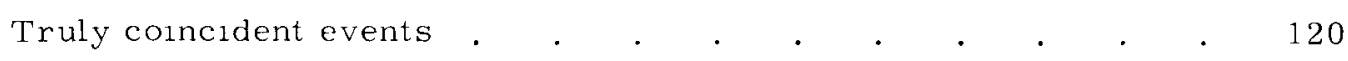

Two-component

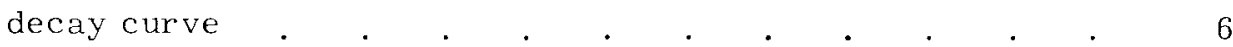

decay curve of a -- system . . . . . . . . . 106

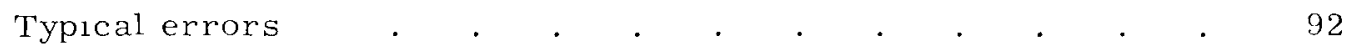

Unantıcıpated

effect on least-squares solutions of any --- varıances. .

Unblased estimate

of variance $\quad$. $\quad$. $\quad$. 42

of variance in least-squares solution $\quad$. . . 56

of variance of points in least-squares solution . 53

Uncorrelated

individual quanitles which are to be averaged are not -- 45

variates . . . . . . 35

Unknown

comparison of the decay of the --- species with that of a species with precisely known hall-life . . . . 103

multicomponent systems . . . . . . 109

treatment of -- system by integral transforms . . 110

Unlıke variances

averaging measurements of - - . . . . . . . . . 45

Valid

Poisson distribution not - - . . . . . . . . . . 7 
Page No.

Values:

algebra of expected - . . . . . . . . . . . . 36

averaging replicate - - . . . . . . . . . . . 98

distribution of expected -- for a given observed count for a

large sample . . . . . . . . . . . . . 68

distribution of expected -.. for a given observed count for a

small sample . . . . . . . . . . 69

improving the --- of the input lambda . . . . . . 92

systematic effect on resolution caused by slight variations

in the -- of the lambdas . . . . . . . 84

Variance:

additive --- due to counting equipment . . . . . . $\quad$. 86

analysis of -. . . . . . . . . . . . . . 35,95

average having the least -. . . . . . . . . . . . 45

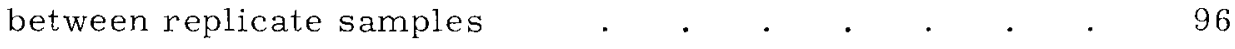

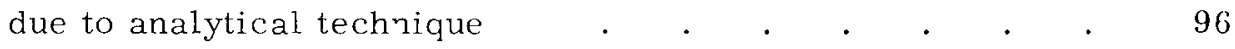

due to counter variation . . . . . . . . . . . . 87

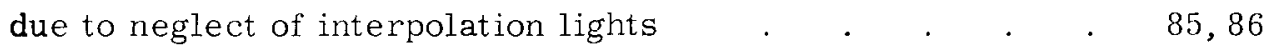

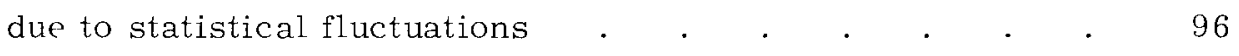

estimation of instrumental - - . . . . . . . . . 88

estimation of population -.. from a sample . . . . 41

estimation of -.- due to instrumental variation . . . . 87

expected value of $\mathrm{n}$ and its --- where we have an actual
observed value of $n$

extrapolation - - . . . . . . . . . . . . . 82

magnitude of instrumental variance $\quad . \quad$. $\quad . \quad . \quad . \quad 88$

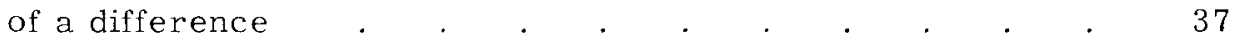

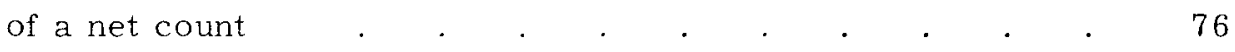

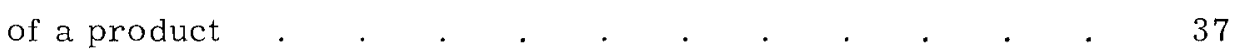

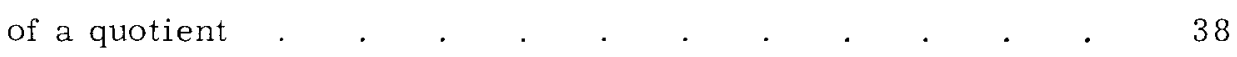

of a sum . . . . . . . . . . . . . . . . 37

of a total distribution . . . . . . . . . . . . . 42

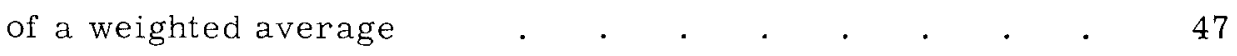

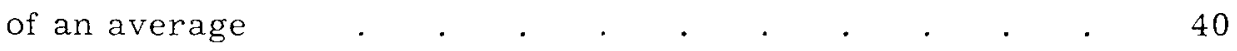

of an extrapolated count rate . . . . . . . . . . 79 
Page No.

Varıance

of an individual measurement $\quad$. $\quad . \quad$. $\quad . \quad$. 40

of an observed count . $\quad . \quad$. . . . . . $\quad$. 84

of any linear combination of variates . $\quad . \quad$. $\quad 39$

of best average value for a given number of replicates . 98

of coincident event rate . . . . 123

of expected value of a count for a Poisson distribution . 69

of expected value of a count for Bernoulis distribution . $\quad 75$

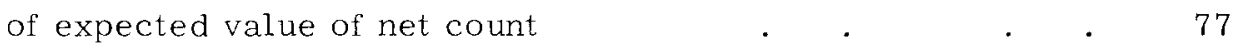

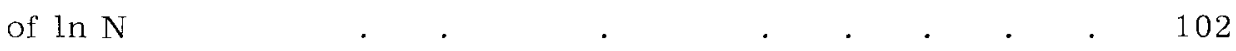

of net counting rate $\quad$. . . . . . . 84

of the distribution formed by a sample . . . . . . 42

of the extrapolation factor . . . . . . . . 81

of the false coincident rate . . . . . . . . . 123

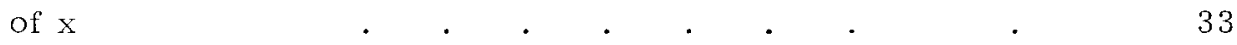

total -.- for each replicate . . . . . . . . . $\quad$. 96

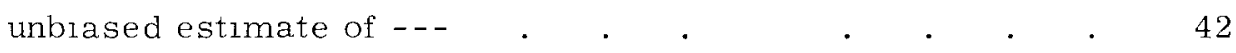

unbiased estimate of -- - in least-squares solution . . 56

unbiased estimate of -- of points in least-squares solution 53

Varıances.

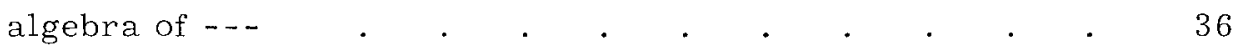

averagıng measurements of unlıke --- . . . . . . 45

diagonal elements of covariance matrix are -- _ . . . . . 87

effect on least-squares solutions of any unanticipated --. . 83

estimate to correct for variances not included in least-
squares weighting

of results of least-squares solutions . . 52

which contribute to the weighting factor . . 83

Variate $\quad$. $\quad$. . . . . . . . $\quad 31$

Varıates

uncorrelated . . . . 35

varıance of any linear combination of --- 39 
Page No.

Variation.

estimation of variance due to instrumental -.- $\quad$. 87

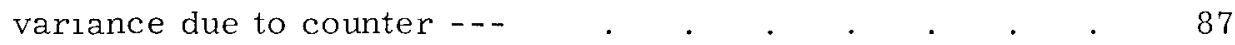

Variations *

analytical

systematic effect on resolution caused by light - - in

the values of the lambdas

Varying

flux

effect of --- an input lambda on the fit of the data to the calculated curve

Vector

column

row

expected value of a matrix or -.-

transpose of a

Vectors

orthogonal -..-

Weight

in the averaging process

Welghted

average

equations for data in matrix form

residual

residuals of the system

sum of the square

variance of a average

Weighting

estimate to correct for variances not included in leastsquares ---

factor to average the replicate results

factors

factors in least-squares solutions 
Page No.

Weighting

proper --- factors for use in a least-squares calculation

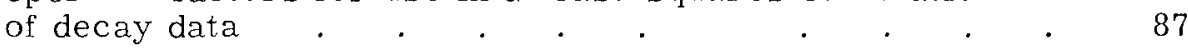
proper -- for least-squares . . . . . . . 50

proper -- of decay data for a least-squares solution . 83

variances which contribute to the --- factor . . . 83

Zero-time

substitution of a spurious --- . . . . . . . . . . . . 994

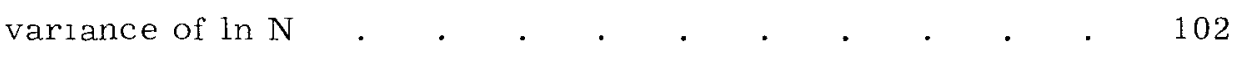




\section{MONOGRAPHS IN THE RADIOCHEMISTRY AND THE RADIOCHEMICAL TECHNIQUE SERIES}

Copies of the following monographs are available from the Clearinghouse for Federal Scientific and Technical Information, National Bureau of Standards, U. S. Department of Commerce, Springfield, Va. 22151

Aluminum and Gallium, NAS-NS-3032, $\$ 0.50$

Americium and Curium, NAS-NS-3006, $\$ 0.75$

Antimony, NAS-NS-3033, $\$ 0.50$

A rsenic, NAS-NS-3002, (Rev.)1965 $\$ 1.00$

Astatine, NAS-NS-3012, $\$ 0.50$

Barium, Calcium, and Strontium, NAS-NS$3010, \$ 1.25$

Beryllium, NAS-NS-3013, \$0.75

Cadmium, NAS-NS-3001, \$0.75

Carbon, Nitrogen, and Oxygen, NAS-NS$3019, \$ 0.50$

Cesium, NAS-NS-3035, \$0.75

Chromuum, NAS-NS-3007, (Rev.)1964 \$0.75

Cobalt, NAS-NS-3041, $\$ 1,00$

Copper, NAS-NS-3027, \$0.75

Fluorine, Chlorine, Bromine, and fodine, NAS-NS-3005, $\$ 0.50$

Francium, NAS-NS-3003, $\$ 0.50$

Germanium, NAS-NS-3043, $\$ 0.50$

Gold, NAS-NS-3036, $\$ 0.50$

Indium, NAS-NS-3014, $\$ 0.50$

Iridium, NAS-NS-3045, $\$ 0.50$

Iron, NAS-NS-3017, $\$ 0.50$

Lead, NAS-NS-3040, \$1.75

Magnesium, NAS-NS-3024, $\$ 0.50$

Manganese, NAS-NS-3018, \$0.50

Mercury, NAS-NS-3026, $\$ 0.50$

Molybdenum, NAS-NS-3009, $\$ 0.50$

Nickel, NAS-NS-3051, $\$ 0.50$

Niobium and Tantalum, NAS-NS-3039, $\$ 0.75$

Osmium, NAS-NS-3046, $\$ 0.50$

Palladium, NAS-NS-3052, $\$ 0.75$

Phosphorus, NAS-NS-3056, $\$ 0.50$

Platinum, NAS-NS-3044, $\$ 0.50$

Plutonium, NAS-NS-3058, $\$ 2.00$

Polonium, NAS-NS-3037, \$0.75

Potassium, NAS-NS-3048, $\$ 0.50$

Protactinium, NAS-NS-3016, \$1.00

Radium, NAS-NS-3057, \$2.25

Rare Earths - Scandium, Yttrium, and AC-

tinium, NAS-NS-3020, $\$ 3.00$

Rare Gases, NAS-NS-3025, $\$ 0.75$

Rhenium, NAS-NS-3028, $\$ 0.50$

Rhodium, NAS-NS-3008, (Rev.) $1965 \$ 1.00$
Rubidium, NAS-NS-3053, $\$ 0.50$

Ruthenium, NAS-NS-3029, $\$ 1.00$

Selenium, NAS-NS-3030, (Rev.) $1965 \$ 1.00$

Silicon, NAS-NS-3049, $\$ 0.50$

Silver, NAS-NS-3047, $\$ 0.75$

Sodium, NAS-NS-3055, $\$ 0.50$

Sulfur, NAS-NS-3054, $\$ 0.50$

Technetium, NAS-NS-3021, $\$ 0.50$

Tellurium, NAS-NS-3038, \$0,50

Thorium, NAS-NS-3004, $\$ 0.75$

Tin, NAS-NS-3023, $\$ 0.75$

Titanium, NAS-NS-3034, $\$ 0.50$

Transcurium Elements, NAS-NS-3031, $\$ 0.50$

Tungsten, NAS-NS-3042, $\$ 0.50$

Uranium, NAS-NS-3050, \$3.50

Vanadium, NAS-NS-3022, \$0.75

Zinc, NAS-NS-3015, $\$ 0.75$

Zirconium and Hafnium, NAS-NS-3011, $\$ 0.50$

Activation Analysis with Charged Particles, NAS - NS - $3110, \$ 1.00$

Applications of Computers to Nuclear and

Radiochemistry, NAS-NS-3107, \$2.50

Application of Distillation Techniques to

Radiochemical Separations, NAS-NS-

$3108, \$ 0.50$

Detection and Measurement of Nuelear $\mathrm{Ra}$ diation, NAS-NS-3105, $\$ 1.50$

Liquid-liquid Extraction with Highmolecular-weight Amines, NAS-NS$3101, \$ 1.00$

Low-level Radiochemical Separations, NASNS-3103, $\$ 0.50$

Paper Chromatographic and Electromigration Techniques in Radiochemistry, NASNS-3106, $\$ 0.50$

Processing of Counting Data, NAS-NS$3109, \$ 1.75$

Rapid Radfochemical Separations, NAS-NS$3104, \$ 1.25$

Separations by Solvent Extraction with Trin-octylphosphine Oxide, NAS-NS-3102, $\$ 0.75$ 